Convergência compacta de resolvente e o teorema de Trotter Kato para perturbações singulares

Cesar Augusto Esteves das Neves Cardoso 



\title{
Convergência compacta de resolvente e o teorema de Trotter Kato para perturbações singulares
}

\section{Cesar Augusto Esteves das Neves Cardoso}

Orientador: Prof. Dr. Alexandre Nolasco de Carvalho

\begin{abstract}
Dissertação apresentada ao Instituto de Ciências Matemáticas e de Computação - ICMC-USP, como parte dos requisitos para obtenção do título de Mestre em Ciências - Matemática . VERSÃO REVISADA
\end{abstract}

\author{
USP - São Carlos
}

Abril de 20012 
Ficha catalográfica elaborada pela Biblioteca Prof. Achille Bassi e Seção Técnica de Informática, ICMC/USP, com os dados fornecidos pelo(a) autor(a)

Cardoso, Cesar Augusto Esteves das Neves
Convergência compacta de resolvente e o teorema
de Trotter Kato para perturbações singulares / Cesar
Augusto Esteves das Neves Cardoso; orientador
Alexandre Nolasco Carvalho. - São Carlos, 2012.
90 p.
Dissertação (Mestrado - Programa de Pós-Graduação en
Matemática) -- Instituto de Ciências Matemáticas e
de Computação, Universidade de São Paulo, 2012.
1. Convergência compacta. 2. Resolvente. 3.
Teorema de Trotter kato. 4. Homogeneização. 5.
Domínios finos. I. Carvalho, Alexandre Nolasco,
orient. II. Título.




\section{Agradecimentos}

Agradeço a Deus, pela saúde, família, amigos e pelas oportunidades que me permitiram chegar até aqui.

Aos meus pais, Ananias e Clarice, por toda a dedicação e apoio de sempre.

A Danielle, meu amor, minha companheira, minha melhor amiga, que me ensinou a ser uma pessoa melhor, para ela com amor e admiração, obrigado Nê.

Aos funcionários e professores do ICMC/USP, em particular, ao professor Alexandre Nolasco, meu orientador, pela orientação, amizade e por sempre estar disposto a me ajudar no trabalho, muito obrigado.

A professora Simone Mazzini, pela amizade e orientação na graduação.

Aos professores membros da banca, Simone Mazzini e Francisco Odair, por aceitarem o convite e pelas sugestões dadas.

Ao Conselho Nacional de Desenvolvimento Científico e Tecnológico - CNPq, pelo apoio financeiro.

De modo geral, agradeço a todos meus amigos e colegas que, direta ou indiretamente, contribuíram para realização deste trabalho. 


\section{Resumo}

Nesta dissertação estudamos uma versão do Teorema de Trotter-Kato que estabelece uma equivalência entre a continuidade, relativamente a um parâmetro, de operadores resolvente e a continuidade dos semigrupos lineares associados. Os operadores ilimitados envolvidos (geradores de semigrupos analíticos) estão definidos em espaços que variam com o parâmetro e isto nos leva a ter que comparar elementos de espaços de Banach diferentes. Este resultado é aplicado a um problema de Neumann em um domínio fino com fronteira altamente oscilante e que se degenera a um intervalo quando o parâmetro varia. Nesta aplicação, utilizamos o método das múltiplas escalas (comum em teoria de homogeneização) para obter formalmente o problema limite (veja [17]) e, em seguida, provamos a convergência compacta dos operadores resolventes utilizando as funções teste oscilantes de Tartar [15], [16] (veja também Cioranescu e Saint Jean Paulin [12]), obtidas através de um problema auxiliar, juntamente com operadores de extensão.

Palavras chave: homogeneização, convergência compacta, semigrupos. 


\section{Abstract}

In this work we study a version of Trotter-Kato's Theorem that establishes an equivalence between the continuity, with respect to a parameter, of the resolvent operators and the continuity of the associated linear semigroups. The unbounded operators involved (generators of analytic semigroups) are defined spaces that vary with the parameter leading us to introduce methods to compare vectors in different Banach spaces. We apply this theorem to an elliptic boundary value problem with Neumann boundary condition in a highly oscillating thin domain that degenerates to a line segment as the parameter varies. In this application we use the multiple scale method (frequently used in the homogenization theory) to obtain, formally, the limiting problem (see [17]) and, in the sequel, we prove the compact convergence of resolvent operators using the oscillating test functions of Tartar [15] (see also [16] and Cioranescu and Saint Jean Paulin [12]) defined with the aid of an auxiliary problem as well as extension operators.

Key words: homogeneization, compact convergence, semigroups. 


\section{Sumário}

$\begin{array}{ll}\text { Introdução } & 3\end{array}$

1 Teoremas de $\boldsymbol{E}$-convergência $\quad 13$

1.1 Operadores Simétricos, Auto-adjuntos, Dissipativos e Imagem Numérica . . . 13

1.2 Continuidade do Espectro . . . . . . . . . . . . . . . . 15

1.3 Potências Fracionárias . . . . . . . . . . . . . . . . . . . . . . 21

1.4 Teoremas de Trotter-Kato . . . . . . . . . . . . . . . . . 23

2 Noções de Homogeneização $\quad 27$

2.1 Introdução à Homogeneização . . . . . . . . . . . . . . . . . . . . . . . . . . . 28

2.2 Funções periódicas altamente oscilantes . . . . . . . . . . . . . . . . . . . . 32

2.3 Espaços de Sobolev . . . . . . . . . . . . . . . . . . . . . . . . . . . . . . 38

2.4 Método de Tartar e o Teorema de Convergência . . . . . . . . . . . . . . . . 40

3 Homogeneização em domínios finos altamente oscilantes 45

3.1 Introdução . . . . . . . . . . . . . . . . . . . . . . . 45

3.2 O Método das Múltiplas Escalas e o Problema Limite . . . . . . . . . . . . . 47

3.3 O Operador Extensão . . . . . . . . . . . . . . . . . . . 51

3.4 Teorema de Convergência para o Limite Homogeneizado . . . . . . . . . . . 54

3.4.1 Funções auxiliares periódicas . . . . . . . . . . . . . 55

3.4 .2 Limite das funções periódicas auxiliares . . . . . . . . . . . . . 59

3.4.3 Convergência para o limite homogeneizado . . . . . . . . . . . 63

3.5 Convergência do Resolvente . . . . . . . . . . . . . . . . . 67

3.6 Convergência dos Semigrupos . . . . . . . . . . . . . . . . 72 
4 Apêndice: estimativa do erro $\quad 77$

4.1 Preliminares . . . . . . . . . . . . . . . . . . . . 78

4.2 Corretor de Primeira Ordem . . . . . . . . . . . . . . . . . 82

4.3 Corretor de Segunda Ordem . . . . . . . . . . . . . . . . 84

$\begin{array}{ll}\text { Considerações finais } & 87\end{array}$ 


\section{Introdução}

No estudo de reações químicas que ocorrem em um recipiente (ou meio), a determinação da forma do recipiente $\Omega_{\epsilon} \subset \mathbb{R}^{3}$ é feita através de medidas e observações que, por sua natureza, contém imprecisões. Se $\Omega_{0}$ denota o recipiente e $\Omega_{\epsilon}$ o seu modelo as funções concentração reais $\phi_{0}: \Omega \rightarrow \mathbb{R}$ e $\phi_{\epsilon}: \Omega_{\epsilon} \rightarrow \mathbb{R}$ estão definidas em espaços diferentes. Mesmo quando o espaço onde atuam os operadores lineares envolvidos pode ser fixado, os operadores (que são determinados por leis empíricas e observações) variam. Assim, para que o modelo estudado reflita (de alguma maneira) o problema modelado, precisamos desenvolver mecanismos de comparar funções pertencentes a espaços diferentes bem como operadores que atuam nestes espaços. Existem inúmeras situações práticas em que somos levados a comparar operadores que atuam em espaços diferentes. Problemas parabólicos em domínios finos ou em domínios do tipo dumbbell e problemas de homogenização são apenas alguns dos exemplos (veja $[1],[2],[3],[4])$.

O restante desta introdução será dedicado a apresentação da terminologia mínima necessária para podermos enunciar os resultados principais da dissertação. Primeiramente introduziremos a noção de $E$-convergência e convergência compacta que nos permitirão comparar vetores em espaços de Banach distintos e com estas noções enunciaremos o um teorema que garante a convergência de auto-valores e auto-funções para uma família de operadores cujo resolvente converge compactamente. Em seguida apresentamos a noção de semigrupos de operadores lineares e suas relações com o resolvente (no caso de semigrupos analíticos) para obter (usando a noção de E-convergência) um Teorema de Trotter-Kato para espaços que variam com parâmetro. Por fim, apresentamos um exemplo de aplicação do Teorema de Trotter-Kato onde o estudo da $E$ - convergência e convergência compacta faz uso de técnicas de teoria de homogenização. 


\section{E-convergência}

A seguir, fazemos uma breve apresentação dos métodos de análise funcional utilizados para tratar problemas em espaços que variam com um parâmetro (veja [6]).

Seja $X_{\epsilon}$ uma família de espaços de Banach, $\epsilon \in[0,1]$, e suponha que existe uma família de operadores lineares contínuos $E_{\epsilon}: X \rightarrow X_{\epsilon}$ com a propriedade

$$
\left\|E_{\epsilon} u\right\|_{X_{\epsilon}} \stackrel{\epsilon \rightarrow 0}{\rightarrow}\|u\|_{X}, \text { para todo } u \in X .
$$

Definição 0.0.1. Diremos que uma sequência $\left\{u_{\epsilon}\right\}_{\epsilon \in(0,1]}$, com $u_{\epsilon} \in X_{\epsilon}$ para todo $\epsilon \in[0,1]$, E-converge para u se $\left\|u_{\epsilon}-E_{\epsilon} u\right\|_{X_{\epsilon}} \stackrel{\epsilon \rightarrow 0}{\longrightarrow} 0$. Escrevemos $u_{\epsilon} \stackrel{E}{\longrightarrow}$ u para dizer que a sequência $\left\{u_{\epsilon}\right\}_{\epsilon \in[0,1]}$ E-converge para u quando $\epsilon$ tende a zero.

Com esta noção de convergência apresentamos as definições de sequência $E$-relativamente compacta, EE-convergência e convergência compacta.

Definição 0.0.2. Uma sequência $\left\{u_{n}\right\}_{n \in \mathbb{N}}$, com $u_{n} \in X_{\epsilon_{n}}$ e $\epsilon_{n} \rightarrow 0$, é dita E-relativamente compacta se, para cada subsequência $\left\{u_{n^{\prime}}\right\}$ de $\left\{u_{n}\right\}$, existe uma subsequência $\left\{u_{n^{\prime \prime}}\right\}$ de $\left\{u_{n^{\prime}}\right\}$ e um elemento $u \in X$ tal que $u_{\epsilon_{n^{\prime \prime}}} \stackrel{E}{\longrightarrow} u$. A família $\left\{u_{\epsilon}\right\}_{\epsilon \in(0,1]}$ é dita E-relativamente compacta se cada sequência $\left\{u_{\epsilon_{n}}\right\}, \epsilon_{n} \rightarrow 0$, é E-relativamente compacta.

Definição 0.0.3. Diremos que a família de operadores $\left\{B_{\epsilon} \in \mathcal{L}\left(X_{\epsilon}\right)\right\}_{\epsilon \in[0,1]}$ EE-converge para $B_{0}$ quando $\epsilon \rightarrow 0$, se $B_{\epsilon} u_{\epsilon} \stackrel{E}{\longrightarrow} B_{0}$ u sempre que $u_{\epsilon} \stackrel{E}{\longrightarrow} u \in X$. Escreveremos $B_{\epsilon} \stackrel{E E}{\longrightarrow} B_{0}$ quando $\epsilon \rightarrow 0$ para denotar que $\left\{B_{\epsilon} \in \mathcal{L}\left(X_{\epsilon}\right)\right\}_{\epsilon \in[0,1]}$ EE-converge para $B_{0}$.

Definição 0.0.4. Uma família de operadores compactos $\left\{B_{\epsilon} \in \mathcal{K}\left(X_{\epsilon}\right): \epsilon \in[0,1]\right\}$ converge compactamente para $B_{0}$ se, para qualquer família $\left\{u_{\epsilon}\right\}$ com $u_{\epsilon} \in X_{\epsilon},\left\|u_{\epsilon}\right\|_{X_{\epsilon}}=1, \epsilon \in(0,1]$, a família $\left\{B_{\epsilon} u_{\epsilon}\right\}_{\epsilon \in[0,1]}$ é E-relativamente compacta e, além disso, $B_{\epsilon} \stackrel{E E}{\longrightarrow} B_{0}$. Escreveremos $B_{\epsilon} \stackrel{C C}{\longrightarrow} B_{0}$ quando $\epsilon \rightarrow 0$ para denotar que $\left\{B_{\epsilon} \in \mathcal{K}\left(X_{\epsilon}\right)\right\}_{\epsilon \in[0,1]}$ converge compactamente para $B_{0}$.

A seguir, apresentamos a definição de resolvente e espectro para um operador linear fechado e a definição de operadores lineares com resolvente compacto.

Definição 0.0.5. Seja $X$ um espaço de Banach sobre $\mathbb{C}$ e $A: D(A) \subset X \rightarrow X$ um operador linear fechado. $O$ conjunto resolvente de $A$ é o subconjunto $\rho(A)$ de todos os $\lambda$ em $\mathbb{C}$ tais que $\lambda-A$ é bijetor. O espectro de $A$ é definido como $\mathbb{C}-\rho(A)$.

Definição 0.0.6. Seja $X$ um espaço de Banach sobre $\mathbb{C}$ e $A: D(A) \subset X \rightarrow X$ um operador fechado e com resolvente não vazio. Um operador linear fechado A tem resolvente compacto se, para algum $\lambda_{0} \in \rho(A),\left(\lambda_{0}-A\right)^{-1} \in \mathcal{K}(X)$. 
Se $A: D(A) \subset X \rightarrow X$ é um operador linear fechado, $\rho(A) \neq \varnothing$ e $\mu, \lambda \in \rho(A)$, é fácil provar que

$$
(\lambda-A)^{-1}-(\mu-A)^{-1}=(\mu-\lambda)(\lambda-A)^{-1}(\mu-A)^{-1} .
$$

Esta identidade é conhecida como a identidade do resolvente.

É uma consequência simples da identidade do resolvente que, se $A$ tem resolvente compacto, então $(\lambda-A)^{-1}$ é compacto para todo $\lambda \in \rho(A)$. Além disso, se $\sigma$ é um conjunto espectral limitado e $P_{\sigma}$ é a projeção espectral associada entao $P_{\sigma}$ é compacta (consequentemente tem imagem com dimensão finita).

Em geral, os operadores $B_{\epsilon}$ serão inversos de certos operadores diferenciais $A_{\epsilon}$. Assim, considere a família de operadores $\left\{A_{\epsilon}: D\left(A_{\epsilon}\right) \subset X_{\epsilon} \rightarrow X_{\epsilon}, \epsilon \in[0,1]\right\}$ e suponha que, para todo $\epsilon \in[0,1]$,

$$
A_{\epsilon} \text { é fechado, tem resolvente compacto, } 0 \in \rho\left(A_{\epsilon}\right) \text {, e } A_{\epsilon}^{-1} \stackrel{C C}{\longrightarrow} A_{0}^{-1} \text {. }
$$

Para cada $\delta>0$ e $\lambda_{0} \in \mathbb{C}$ defina $S_{\delta}\left(\lambda_{0}\right):=\left\{\mu \in \mathbb{C}:\left|\lambda-\lambda_{0}\right|=\delta\right\}$.

A um ponto isolado $\lambda \in \sigma\left(A_{0}\right)$ associamos o seu auto-espaço generalizado $W\left(\lambda, A_{0}\right)=$ $Q\left(\lambda, A_{0}\right) X$ onde

$$
Q\left(\lambda, A_{0}\right)=\frac{1}{2 \pi i} \int_{|\xi-\lambda|=\delta}\left(\xi I-A_{0}\right)^{-1} d \xi
$$

e $\delta$ é escolhido de forma que não haja nenhum outro ponto de $\sigma\left(A_{0}\right)$ no $\operatorname{disco} \bar{B}_{\delta}^{\mathbb{C}}(\lambda)=\{\xi \in$ $\mathbb{C}:|\xi-\lambda| \leqslant \delta\}$. Segue do Lema 1.2 .3 que existe $\epsilon_{S_{\delta}(\lambda)}$ tal que $\rho\left(A_{\epsilon}\right) \supset S_{\delta}(\lambda)$ para todo $\epsilon \leqslant \epsilon_{S_{\delta}(\lambda)}$. Seja $W\left(\lambda, A_{\epsilon}\right):=Q\left(\lambda, A_{\epsilon}\right) X_{\epsilon}$ onde

$$
Q\left(\lambda, A_{\epsilon}\right)=\frac{1}{2 \pi i} \int_{|\xi-\lambda|=\delta}\left(\xi I-A_{\epsilon}\right)^{-1} d \xi
$$

O resultado a seguir diz que o espectro de $A_{\epsilon}$, para $\epsilon$ pequeno, se aproxima do espectro de $A_{0}$ quando $\epsilon$ tende a zero. Sabe-se que o espectro de $A_{\epsilon}$ ou $A_{0}$ contém apenas auto-valores isolados de multiplicidade finita (recorde que estes operadores estão definidos em espaços distintos).

Teorema 0.0.1. Seja $\left\{A_{\epsilon}: D\left(A_{\epsilon}\right) \subset X_{\epsilon} \rightarrow X_{\epsilon}, \epsilon \in[0,1]\right\}$ uma família de operadores tal que (2) está satisfeita. Então valem as seguintes afirmativas:

(i) Se $\lambda_{0} \in \sigma\left(A_{0}\right)$, existe seqüência $\left\{\epsilon_{n}\right\}$ em $(0,1]$ com $\epsilon_{n} \stackrel{n \rightarrow \infty}{\longrightarrow} 0$ e sequência $\left\{\lambda_{n}\right\}$ em $\mathbb{C}$ com $\lambda_{n} \in \sigma\left(A_{\epsilon_{n}}\right)$, para $n=1,2,3 \cdots$, e $\lambda_{n} \stackrel{n \rightarrow \infty}{\longrightarrow} \lambda_{0}$. 
(ii) Se $\left\{\epsilon_{n}\right\}$ é uma sequência em $(0,1]$ com $\epsilon_{n} \stackrel{n \rightarrow \infty}{\longrightarrow} 0, e\left\{\lambda_{n}\right\}$ é uma sequência em $\mathbb{C}$ com $\lambda_{n} \in \sigma\left(A_{\epsilon_{n}}\right), n \in \mathbb{N}$ e $\lambda_{n} \stackrel{n \rightarrow \infty}{\longrightarrow} \lambda_{0}$, então $\lambda_{0} \in \sigma\left(A_{0}\right)$.

Além disso, se existe $\epsilon_{0} \in(0,1]$ tal que $\sup _{\epsilon \in\left[0, \epsilon_{0}\right]}\left\|E_{\epsilon}\right\|_{\mathcal{L}\left(X_{0}, X_{\epsilon}\right)}<\infty$, valem as seguintes afirmativas:

(iii) Existe $\epsilon_{1} \in\left(0, \epsilon_{0}\right)$ tal que $\operatorname{dim} W\left(\lambda, A_{\epsilon}\right)=\operatorname{dim} W\left(\lambda_{0}, A_{0}\right)$ para todo $0 \leqslant \epsilon \leqslant \epsilon_{1}$.

(iv) Se $u \in W\left(\lambda_{0}, A_{0}\right)$, então existe uma sequência $\left\{\epsilon_{n}\right\}$ em $(0,1] \operatorname{com} \epsilon_{n} \stackrel{n \rightarrow \infty}{\longrightarrow} 0, u_{\epsilon_{n}} \in$ $W\left(\lambda_{0}, A_{\epsilon_{n}}\right)$ e tal que $u_{\epsilon_{n}} \stackrel{E}{\longrightarrow}$ u quando $n \rightarrow \infty$.

(v) Se $\left\{\epsilon_{n}\right\}$ é uma sequência em $(0,1]$ com $\epsilon_{n} \stackrel{n \rightarrow \infty}{\longrightarrow} 0$, e $\left\{u_{n}\right\}$ é uma sequência com $u_{n} \in$ $W\left(\lambda, A_{\epsilon_{n}}\right),\left\|u_{n}\right\|_{X_{\epsilon_{n}}}=1$, então $\left\{u_{n}\right\}$ tem uma subsequência E-convergente para um vetor $u$ in $W\left(\lambda_{0}, A_{0}\right)$.

\section{Teoremas de Trotter-Kato}

Chamaremos de Teoremas de Trotter-Kato aos teoremas que descrevem alguma relação entre a convergência do resolvente e a convergência dos semigrupos gerados pelos operadores associados. A seguir, definimos a noção de semigrupo e enunciamos os teoremas que utilizamos ao demonstrar um Teorema de Trotter-Kato (Teorema 0.0.5) para sequências de operadores definidos em espaços que variam com o parâmetro da sequência.

Definição 0.0.7. Um semigrupo de operadores lineares em $X$ é uma família $\{T(t): t \geq$ $0\} \subset \mathcal{L}(X)$ tal que tal que

(i) $T(0)=I_{X}$,

(ii) $T(t+s)=T(t) T(s)$, para todo $t, s \geq 0$.

Além disso,

(iii) se $\left\|T(t)-I_{X}\right\|_{\mathcal{L}(X)} \rightarrow 0$ quando $t \rightarrow 0^{+}$, dizemos que o semigrupo é uniformemente contínuo

(iv) se $\|T(t) x-x\|_{X} \rightarrow 0$ quando $t \rightarrow 0^{+}, \forall x \in X$, dizemos que o semigrupo é fortemente contínuo.

Definição 0.0.8. Se $\{T(t), t \geq 0\} \subset \mathcal{L}(X)$ é um semigrupo fortemente contínuo de operadores lineares, seu gerador infinitesimal é o operador definido por $A: D(A) \subset X \rightarrow X$, 
onde

$$
\begin{array}{r}
D(A)=\left\{x \in X: \lim _{t \rightarrow 0^{+}} \frac{T(t) x-x}{t} \text { existe }\right\}, \\
A x=\lim _{t \rightarrow 0^{+}} \frac{T(t) x-x}{t}, \quad \forall x \in D(A) .
\end{array}
$$

Teorema 0.0.2. Suponha que $\{T(t), t \geq 0\} \subset \mathcal{L}(X)$ é um semigrupo fortemente contínuo. Então, existe $M \geq 1$ e $\beta$ tais que

$$
\|T(t)\|_{\mathcal{L}(X)} \leq M \mathrm{e}^{\beta t}, \quad \forall t \geq 0 .
$$

Para qualquer $\ell>0$ podemos escolher $\beta \geq \frac{1}{\ell} \log \|T(\ell)\|_{\mathcal{L}(X)}$ e então escolher $M$.

Teorema 0.0.3. Se $\{T(t), t \geq 0\} \subset \mathcal{L}(X)$ é um semigrupo fortemente contínuo, $\beta$ é o número real dado no Teorema 0.0.2 e $\lambda \in \mathbb{C}$ é tal que $\operatorname{Re} \lambda>\beta$, então $\lambda \in \rho(A)$ e

$$
(\lambda-A)^{-1} x=\int_{0}^{\infty} \mathrm{e}^{-\lambda t} T(t) x d t, \quad \forall x \in X
$$

Teorema 0.0.4. Suponha que $A: D(A) \subset X \rightarrow X$ é densamente definido $e-A$ é setorial; isto é, existem constantes a, $C$ e $\varphi \in(\pi / 2, \pi], \Sigma_{a, \varphi}=\{\lambda \in \mathbb{C}:|\arg (\lambda-a)|<\varphi\} \subset \rho(A) e$

$$
\left\|(\lambda-A)^{-1}\right\|_{\mathcal{L}(X)} \leq C /|\lambda-a|, \quad \forall \lambda \in \Sigma_{a, \varphi}
$$

Então $A$ é o gerador infinitesimal de um semigrupo fortemente contínuo $\{T(t), t \geq 0\} \subset$ $\mathcal{L}(X)$ com

$$
T(t)=\frac{1}{2 \pi i} \int_{\Gamma_{a}} \mathrm{e}^{\lambda t}(\lambda-A)^{-1} d \lambda
$$

onde $\Gamma_{a}$ é a fronteira de $\Sigma_{a, \phi} \backslash\{\lambda \in \mathbb{C}:|\lambda-a| \leq r\}, \frac{\pi}{2}<\phi<\varphi, r$ pequeno, orientada com no sentido da parte imaginária crescente. Além disso, $t \mapsto T(t)$ se estende a uma função analítica de $\{t \in \mathbb{C}:|\arg t|<\phi-\pi / 2\}$ em $\mathcal{L}(X)$ (ou a complexificação de $X$, se $X$ é um espaço de Banach real) e para algum $K=K\left(\Gamma_{a}, C\right)>0$

$$
\|T(t)\|_{\mathcal{L}(X)} \leq K \mathrm{e}^{a t}, \quad\|A T(t)\|_{\mathcal{L}(X)} \leq K t^{-1} \mathrm{e}^{a t}
$$

para todo $t>0$. Note que

$$
\frac{d}{d t} T(t)=A T(t)
$$

é um operador limitado para qualquer $t>0$.

Neste estudo, estaremos interessados em verificar para uma certa classe de problemas as hipóteses do seguinte Teorema de Trotter-Kato. 
Teorema 0.0.5. Sejam $A: X \supset D(A) \rightarrow X, A_{n}: X_{n} \supset D\left(A_{n}\right) \rightarrow X_{n}, n \in \mathbb{N}$ operadores lineares densamente definidos tais que

$$
\left\|\left(\lambda I_{n}-A_{n}\right)^{-1}\right\| \leq \frac{M}{|\lambda-\omega|}, \forall \lambda \in \Sigma_{\omega, \varphi} \forall n \in \mathbb{N} .
$$

para algum setor $\Sigma_{\omega, \varphi}:=\{\lambda \in \mathbb{C} ;|\arg (\lambda-\omega)| \leq \varphi\}, \varphi \in\left(\frac{\pi}{2}, \pi\right)$. Então as seguintes condições são equivalentes:

1. Existe $\lambda \in \mathbb{C}$ com $\operatorname{Re}(\lambda)>\omega$ tal que

$$
\left(\lambda I_{n}-A_{n}\right)^{-1} \stackrel{E E}{\rightarrow}(\lambda I-A)^{-1}
$$

2. Existe $0<\theta<\frac{\pi}{2}$ tal que $\forall \epsilon_{0}, \mu \in(0, \infty), \epsilon_{0}<\mu$ temos

$$
\sup _{t \in \Sigma\left(\theta, \epsilon_{0}, \mu\right)}\left\|e^{A_{n} t} u_{n}-E_{n} e^{A t} u_{0}\right\|_{X_{n}} \stackrel{n \rightarrow 0}{\rightarrow} 0
$$

sempre que $u_{n} \stackrel{E}{\rightarrow} u_{0}$, onde

$$
\Sigma\left(\theta, \epsilon_{0}, \mu\right)=\left\{z \in \mathbb{C} ;|\arg (z)| \leq \theta \text { e } \epsilon_{0} \leq|t| \leq \mu\right\} .
$$

Isto é, $e^{A_{n} t} \stackrel{E E}{\rightarrow} e^{A t}$ uniformemente para $t$ em compactos $\Sigma\left(\theta, \epsilon_{0}, \mu\right)$.

Em ambos os casos, temos EE-convergência uniforme em compactos contidos em $\Sigma\left(\theta, \epsilon_{0}, \mu\right)$.

\section{Aplicação a um problema de homogeneização em domínios finos}

Aplicaremos o Teorema de Trotter-Kato 0.0.5 para obter propriedades de convergência do semigrupo gerado pelo operador de Laplace com condição de fronteira de Neumann em uma família de domínios $R^{\epsilon} \subset \mathbb{R}^{2}, \epsilon \in[0,1]$, com comportamento altamente oscilatório na fronteira e que se degenera a um segmento de reta quando $\epsilon$ tende a zero. Para isto mostraremos a convergência compacta do resolvente utilizando técnicas de homogeneização.

No que segue, estabelecemos o problema com sua devida notação. Considere o seguinte conjunto

$$
R^{\epsilon}=\left\{(x, y) \in \mathbb{R}^{2} ; 0<x<1 \text { e } 0<y<\epsilon g(x / \epsilon)\right\}
$$

onde $\epsilon>0$ é arbitrário.

Seja $f: \mathbb{R} \rightarrow \mathbb{R}$ uma função L-periódica e de classe $C^{1}$ com

$$
0<g_{0} \leq g(x) \leq g_{1} \forall x \in \mathbb{R},
$$


onde $g_{1}=\max \{g(x) ; x \in \mathbb{R}\}$ e $g_{0}=\min \{g(x) ; x \in \mathbb{R}\}$

$R^{\epsilon}$ é um domínio fino que degenera para um intervalo da reta quando $\epsilon$ tende a zero. Note que $R^{\epsilon}$ tem espessura, período das oscilações e amplitude das oscilações de ordem $\epsilon$.

Nesta dissertação estudamos o comportamento assintótico (quando $\epsilon \rightarrow 0$ ) da família de soluções dos problemas elípticos

$$
\left\{\begin{array}{c}
-\Delta u^{\epsilon}+u^{\epsilon}=f\left(u^{\epsilon}\right) \text { em } R^{\epsilon} \\
\frac{\partial u^{\epsilon}}{\partial \nu^{\epsilon}} \text { sobre } \partial R^{\epsilon}
\end{array}\right.
$$

onde $\nu^{\epsilon}$ é o vetor normal unitário exterior a $\partial R^{\epsilon}$.

Pode-se verificar que o problema (6) é equivalente ao seguinte problema

$$
\left\{\begin{array}{c}
-\frac{\partial^{2} u^{\epsilon}}{\partial x_{1}^{2}}-\frac{1}{\epsilon^{2}} \frac{\partial^{2} u^{\epsilon}}{\partial x_{2}^{2}}+u^{\epsilon}=f\left(u^{\epsilon}\right) \text { em } \Omega^{\epsilon} \\
\frac{\partial u^{\epsilon}}{\partial x_{1}} N_{1}^{\epsilon}+\frac{1}{\epsilon^{2}} \frac{\partial u^{\epsilon}}{\partial x_{2}} N_{2}^{\epsilon}=0 \text { sobre } \partial \Omega^{\epsilon}
\end{array}\right.
$$

onde

$$
\Omega^{\epsilon}=\left\{\left(x_{1}, x_{2}\right) \in \mathbb{R}^{2} ; 0<x_{1}<1 \text { e } 0<x_{2}<g\left(x_{1} / \epsilon\right)\right\}
$$

e $N^{\epsilon}=\left(N_{1}^{\epsilon}, N_{2}^{\epsilon}\right)$ é o vetor normal, unitário exterior a $\partial \Omega^{\epsilon}$.

Neste estudo analisamos o comportamento das soluções do problema (7) modificado da seguinte maneira

$$
\left\{\begin{array}{c}
-\frac{\partial^{2} u^{\epsilon}}{\partial x_{1}^{2}}-\frac{1}{\epsilon^{2}} \frac{\partial^{2} u^{\epsilon}}{\partial x_{2}^{2}}+V^{\epsilon} u^{\epsilon}=f^{\epsilon} \text { em } \Omega^{\epsilon} \\
\frac{\partial u^{\epsilon}}{\partial x_{1}} N_{1}^{\epsilon}+\frac{1}{\epsilon^{2}} \frac{\partial u^{\epsilon}}{\partial x_{2}} N_{2}^{\epsilon}=0 \text { sobre } \partial \Omega^{\epsilon}
\end{array}\right.
$$

Assumimos que $V^{\epsilon} \in L^{\infty}\left(\Omega^{\epsilon}\right), V^{\epsilon} \geq 1$, que existe $V_{0} \in L^{\infty}(\Omega)$, independente da variável $x_{2}$; isto é, $V_{0}\left(x_{1}, x_{2}\right)=V_{0}\left(x_{1}\right)$ e que existe $p>1$ tal que

$$
\sup _{\epsilon \in[0,1]}\left\|V^{\epsilon}\right\|_{L^{\infty}\left(\Omega^{\epsilon}\right)}<\infty, \quad\left\|V^{\epsilon}-V_{0}\right\|_{L^{1}\left(\Omega^{\epsilon}\right)} \rightarrow 0 .
$$

com

$$
\Omega=\left\{\left(x_{1}, x_{2}\right) \in \mathbb{R}^{2} ; x_{1} \in I \text { e } 0<x_{2}<g_{1}\right\}
$$

onde $I=(0,1)$. Assumimos também que $f^{\epsilon} \in L^{2}\left(\Omega^{\epsilon}\right)$ e satisfaz $\left\|f^{\epsilon}\right\|_{L^{2}\left(\Omega^{\epsilon}\right)} \leq C$, onde $C$ é independente de $\epsilon$.

Os operadores que estudamos foram definidos de modo abstrato utilizando (8) em sua formulação variacional, isto é, multiplicando (8) por funções teste (neste caso, funções em $\left.H^{1}\left(\Omega^{\epsilon}\right)\right)$ e integrando por partes. Fazendo isto obtemos 


$$
\int_{\Omega^{\epsilon}}\left\{\frac{\partial u}{\partial x_{1}} \frac{\partial v}{\partial x_{1}}+\frac{1}{\epsilon^{2}} \frac{\partial u}{\partial x_{2}} \frac{\partial v}{\partial x_{2}}+V^{\epsilon} u v\right\} d x_{1} d x_{2}=\int_{\Omega^{\epsilon}} f^{\epsilon} v d x_{1} d x_{2}, \forall v \in H^{1}\left(\Omega^{\epsilon}\right) .
$$

Assim, definimos os operadores $L_{\epsilon}$ da seguinte forma:

$$
\begin{gathered}
\mathcal{D}\left(L_{\epsilon}\right)=\left\{u \in H^{1}\left(\Omega^{\epsilon}\right) ; \exists f \in L^{2}\left(\Omega^{\epsilon}\right) \text { satisfazendo } a_{\epsilon}(u, \varphi)=(f, \varphi)_{\epsilon}, \forall \varphi \in H^{1}\left(\Omega^{\epsilon}\right)\right\} \\
L_{\epsilon} u=f \in L^{2}\left(\Omega^{\epsilon}\right) \text { se } a_{\epsilon}(u, \varphi)=(f, \varphi)_{\epsilon}, \forall \varphi \in H^{1}\left(\Omega^{\epsilon}\right),
\end{gathered}
$$

onde $a_{\epsilon}: H^{1}\left(\Omega^{\epsilon}\right) \times H^{1}\left(\Omega^{\epsilon}\right) \rightarrow \mathbb{R}$ é uma forma bilinear, contínua, coerciva e simétrica definida por

$$
a_{\epsilon}(u, v)=\int_{\Omega^{\epsilon}}\left\{\frac{\partial u}{\partial x_{1}} \frac{\partial v}{\partial x_{1}}+\frac{1}{\epsilon^{2}} \frac{\partial u}{\partial x_{2}} \frac{\partial v}{\partial x_{2}}+V^{\epsilon} u v\right\} d x_{1} d x_{2} .
$$

e $(\cdot, \cdot)_{\epsilon}$ é o produto interno usual de $L^{2}\left(\Omega^{\epsilon}\right)$ dado por

$$
(u, v)_{\epsilon}:=\int_{\Omega^{\epsilon}} u v d x_{1} d x_{2}, \forall u, v \in L^{2}\left(\Omega^{\epsilon}\right) .
$$

Nas condições acima estudamos a convergência das soluções $u^{\epsilon} \in H^{1}\left(\Omega^{\epsilon}\right)$ da seguinte equação

$$
L_{\epsilon} u^{\epsilon}=f^{\epsilon}
$$

Através do método da múltipla escala obtemos $L_{0}: \mathcal{D}\left(L_{0}\right) \subset L^{2}(0,1) \rightarrow L^{2}(0,1)$ dado por

$$
\begin{gathered}
\mathcal{D}\left(L_{0}\right)=\left\{u \in H^{1}(0,1) ; \exists f \in L^{2}(0,1) \text { satisfazendo } a_{0}(u, \varphi)=(f, \varphi)_{0}, \forall \varphi \in H^{1}(0,1)\right\} \\
L_{0} u=f \in L^{2}(0,1) \text { se } a_{0}(u, \varphi)=(f, \varphi)_{0}, \forall \varphi \in H^{1}(0,1)
\end{gathered}
$$

onde $q_{0}>0$ é dado por $(3.60)$ e $a_{0}: H^{1}(0,1) \times H^{1}(0,1) \rightarrow \mathbb{R}$ é definido por

$$
a_{0}(u, v)=\int_{0}^{1}\left(q_{0} \frac{d u}{d x} \frac{d v}{d x}+V_{0} u v\right) d x, \forall u, v \in H^{1}(0,1)
$$

e

$$
(u, v)_{0}:=\int_{0}^{1} u v d x, \forall u, v \in L^{2}(0,1) .
$$

Usando uma ideia de D. Cioranescu and J. Saint J. Paulin que faz uso de operadores extensão, combinado com o método de Tartar das funções-teste oscilantes, obtêm-se o Teorema 3.4.1 de convergência das soluções $u^{\epsilon}$ de (11) para $u_{0}$ que satisfaz $L_{0} u_{0}=f_{0}$ para certa $f_{0} \in L^{2}(0,1)$. Com este teorema obtemos

$$
L_{\epsilon}^{-1} \stackrel{C C}{\rightarrow} L_{0}^{-1}
$$


Disto (e com o auxílio da imagem numérica de $L_{\epsilon}$ e dos teoremas de imersão de Sobolev) obtemos

$$
L_{\epsilon} \text { é fechado, tem resolvente compacto, } 0 \in \rho\left(L_{\epsilon}\right) \text {, e } L_{\epsilon}^{-1} \stackrel{C C}{\longrightarrow} L_{0}^{-1} \text {. }
$$

e portanto segue a convergência dos auto-valores e auto-vetores dadas pelo Teorema 0.0.1.

Continuando, note que a imagem numérica dos operadores $-L_{\epsilon}$ estão contidas em $(-\infty,-1]$ para todo $\epsilon>0$. Assim, segue do Teorema 0.0.4 que existem $M>0$ e $\frac{\pi}{2}<\phi<\pi$, independente de $\epsilon$ tal que

$$
\left\|\left(\mu+L_{\epsilon}\right)^{-1}\right\|_{\mathcal{L}\left(Z_{\epsilon}\right)} \leq \frac{M}{|\mu+1|} \forall \mu \in \Sigma_{-1, \phi}
$$

onde $\Sigma_{-1, \phi}=\{\mu \in \mathbb{C}: 0<|\arg (\mu+1)| \leq \phi\}$.

Por outro lado, de $(12)$ e com o auxílio das projeções $M_{\epsilon}$ e $\widetilde{M}_{\epsilon}$ definidas na seção 3.5 mostramos que

$$
\left(\mu+L_{\epsilon}\right)^{-1} \stackrel{E E}{\rightarrow}\left(\mu+L_{0}\right)^{-1}, \forall \mu \in \Sigma_{-1, \phi}
$$

logo segue do Teorema de Trotter-Kato (Teorema 1.4.4) que existe $0<\theta<\frac{\pi}{2}$ tal que $\forall \epsilon_{0}, \mu \in(0, \infty), \epsilon_{0}<\mu$ temos

$$
\sup _{t \in \Sigma\left(\theta, \epsilon_{0}, \mu\right)}\left\|e^{-L_{\epsilon} t} u_{n}-E_{\epsilon} e^{-L_{0} t} u_{0}\right\|_{Z_{\epsilon}} \stackrel{\epsilon \rightarrow 0}{\rightarrow} 0
$$

sempre que $u_{n} \stackrel{E}{\rightarrow} u_{0}$, onde

$$
\Sigma\left(\theta, \epsilon_{0}, \mu\right)=\left\{z \in \mathbb{C}:|\arg (z)| \leq \theta \text { e } \epsilon_{0} \leq|t| \leq \mu\right\}
$$

isto é, $e^{-L_{\epsilon} t} \stackrel{E E}{\rightarrow} e^{-L_{0} t}$ uniformemente em compactos $\Sigma\left(\theta, \epsilon_{0}, \mu\right)$.

\section{Resumo dos capítulos}

No Capítulo 1 apresentamos uma teoria abstrata de operadores lineares para se analisar a convergência de auto-valores e auto-vetores dos operadores diferencias associados ao problema (7). Apresentamos as noções de resolvente, convergência compacta, potências fracionárias de operadores positivos e finalizamos o capítulo com os teoremas de Trotter-Kato, teoremas que caracterizam quando há equivalencia entre convergência de resolvente e convergência de semigrupos.

No Capítulo 2 apresentamos uma pequena introdução à homogeneização, seus temas de estudo e suas questões. Apresentamos também um teorema sobre convergência fraca e 
fraca* de funções periódicas definidas em $L^{p}(\Omega), 1 \leq p \leq \infty, \Omega \subset \mathbb{R}^{n}$ limitado. Finalizamos o capítulo com uma breve descrição do método de Tartar das funções oscilantes, cujas idéias são fundamentais para se demonstrar o principal teorema de convergência das soluções de (7) (Teorema 3.4.1). Apresentamos também um esboço da demonstração de um teorema clássico de convergência de soluções de equações diferencias para o limite homogeneizado, baseado nas idéias de Tartar.

No Capítulo 3 faremos uso das noções e resultados estabelecidos no capítulo 2 para se analisar convergência das soluções (em algum sentido a ser especificado) do problema (7) Para isto apresentamos direto no problema de interesse o método da múltipla escala (comum em teoria da homogeneização) para se obter formalmente o problema limite. Depois construímos um operador extensão que nos auxiliará na transformação do problema (7) numa equação integral com um domínio fixo, essencial para os cálculos posteriores que demonstram o teorema de convergência 3.4.1. Para se demonstrar o teorema anterior, faremos uso da solução de um problema auxiliar periódico e é a partir deste ponto que faremos uso das idéias de Tartar para se contornar o problema de analisar a convergência da integral de sequências fracamente convergêntes em $L^{2}$, problema que aparece ao se estudar a convergência da formulação variacional de (7).

Depois de estabelecer o Teorema 3.4.1, faremos uso deste para se analisar a convergência compacta dos operadores resolventes associados aos operadores lineares diferenciais definidos à partir de (7). Com a convergência dos resolventes podemos estabelecer a convergência dos semigrupos lineares associados. Faremos isto de duas formas. Uma delas será obtida com o auxílio das famílias de 'projeções' lineares $\left\{M_{\epsilon}\right\}_{\epsilon \in(0,1)}$ e $\left\{\widetilde{M}_{\epsilon}\right\}_{\epsilon \in(0,1)}$ convenientemente definidas combinado com um teorema de operadores setoriais e um teorema potências fracionárias de operadores positivos. A outra forma apresentada é consequência do Teorema de Trotter-Kato (Teorema 0.0.5).

Finalizamos a dissertação com um apêndice sobre a estimativa do erro (em norma) que se comete ao aproximar as soluções $u^{\epsilon}$ do problema (7) pelo truncamento da sua expansão assintótica obtida através do método da múltipla escala. Esta análise é particularmente interessante pois a convergência fraca das soluções obtida no Teorema 3.4.1 é a melhor convergência que se pode obter. Em geral não temos convergência forte das soluções (na norma usual de $H^{1}$ ). 


\section{Capítulo 1}

\section{Teoremas de $\boldsymbol{E}$-convergência}

Neste capítulo vamos apresentar alguns resultados básicos de análise funcional e resultados de $E$-convergência e convergência compacta com o objetivo de mostrar um resultado sobre a convergência do espectro de uma família de operadores que converge compactamente e um Teorema de Trotter-Kato.

\subsection{Operadores Simétricos, Auto-adjuntos, Dissipativos e Imagem Numérica}

Seja $X$ um espaço de Banach com dual $X^{*}$. Se $x^{*} \in X^{*}$ denotaremos o seu valor em um vetor $x \in X$ por $\left\langle x^{*}, x\right\rangle$ ou por $\left\langle x, x^{*}\right\rangle$. Seja $S: D(S) \subset X \rightarrow X$ um operador linear com domínio denso. O adjunto $S^{*}: D\left(S^{*}\right) \subset X^{*} \rightarrow X^{*}$ de $S$ é o operador linear definido por: $D\left(S^{*}\right)$ é o conjunto dos $x^{*} \in X^{*}$ para os quais existe $y^{*} \in X^{*}$ satisfazendo

$$
\left\langle x^{*}, S x\right\rangle=\left\langle y^{*}, x\right\rangle \quad \forall x \in D(S) .
$$

Se $x^{*} \in D\left(S^{*}\right)$ definimos $S^{*} x^{*}:=y^{*}$ onde $y^{*}$ é o (único) elemento de $X^{*}$ satisfazendo (1.1).

Definição 1.1.1. Seja $H$ um espaço de Hilbert sobre $\mathbb{K}$ com produto interno $\langle\cdot, \cdot\rangle$. Dizemos que um operador $A: D(A) \subset H \rightarrow H$ é simétrico (também chamado Hermitiano quando $\mathbb{K}=\mathbb{C}$ ) se $\overline{D(A)}=H$ e $A \subset A^{*}$; isto é, $\langle A x, y\rangle=\langle x, A y\rangle$ para todo $x, y \in D(A)$. Dizemos que $A$ é auto-adjunto se $A=A^{*}$.

Com esta definição, apresentamos o seguinte teorema.

Teorema 1.1.1. Seja $H$ um espaço de Hilbert sobre $\mathbb{K}$ com produto interno $\langle\cdot, \cdot\rangle$. Se A : $D(A) \subset H \rightarrow H$ é um operador simétrico e sobrejetor, então A é auto-adjunto. 
A seguir definimos a noção de dissipatividade.

Definição 1.1.2. Seja $X$ um espaço de Banach sobre $\mathbb{K}$. A aplicação dualidade $J: X \rightarrow 2^{X^{*}}$ é uma função multívoca definida por

$$
J(x)=\left\{x^{*} \in X^{*}: \operatorname{Re}\left\langle x^{*}, x\right\rangle=\|x\|^{2},\left\|x^{*}\right\|=\|x\|\right\} .
$$

$J(x) \neq \emptyset$, pelo Teorema de Hahn-Banach.

Um operador linear $A: D(A) \subset X \rightarrow X$ é dissipativo se para cada $x \in D(A)$ existe $x^{*} \in J(x)$ tal que $\operatorname{Re}\left\langle x^{*}, A x\right\rangle \leq 0$.

Com esta definição temos o seguinte teorema.

Teorema 1.1.2. Seja A um operador linear fechado e densamente definido. Se ambos $A$ e $A^{*}$ são dissipativos, então $\rho(A) \supset(0, \infty)$ e

$$
\left\|(\lambda-A)^{-1}\right\| \leq \frac{1}{\lambda}, \forall \lambda>0 .
$$

Se $A$ é um operador linear em um espaço de Banach complexo $X$ a sua imagem numérica $W(A)$ é o conjunto

$$
W(A):=\left\{\left\langle x^{*}, A x\right\rangle: x \in D(A), x^{*} \in X^{*},\|x\|=\left\|x^{*}\right\|=1,\left\langle x^{*}, x\right\rangle=1\right\} .
$$

No caso em que $X$ é um espaço de Hilbert

$$
W(A)=\{\langle A x, x\rangle: x \in D(A),\|x\|=1\} .
$$

O teorema a seguir é uma importante ferramenta para o estudo de operadores setoriais.

Teorema 1.1.3. Seja $A: D(A) \subset X \rightarrow X$ um operador fechado densamente definido. Seja $W(A)$ a imagem numérica de $A$ e $\Sigma$ um subconjunto aberto e conexo em $\mathbb{C} \backslash W(A)$. Se $\lambda \notin \overline{W(A)}$ então $\lambda-A$ é injetora e tem imagem fechada e satisfaz

$$
\|(\lambda-A) x\| \geq d(\lambda, W(A))\|x\| .
$$

Além disso, se $\rho(A) \cap \Sigma \neq \emptyset$, então $\rho(A) \supset \Sigma e$

$$
\left\|(\lambda-A)^{-1}\right\|_{\mathcal{L}(X)} \leq \frac{1}{d(\lambda, W(A))}, \quad \forall \lambda \in \Sigma .
$$

onde $d(\lambda, W(A))$ é a distância de $\lambda$ a $W(A)$.

Como consequência temos o seguinte corolário. 
Corolário 1.1.1. Seja $H$ um espaço de Hilbert sobre $\mathbb{K}$ e $A: D(A) \subset H \rightarrow H$ um operador auto-adjunto. Segue que A é fechado e densamente definido. Se A é limitado superiormente; isto é, $\langle A u, u\rangle \leq a\langle u, u\rangle$ para algum $a \in \mathbb{R}$, então $\mathbb{C} \backslash(-\infty, a] \subset \rho(A)$, e

$$
\left\|(A-\lambda)^{-1}\right\|_{\mathcal{L}(X)} \leq \frac{M}{|\lambda-a|},
$$

para alguma constante $M \geq 1$ dependendo somente de $\varphi$ e para todo $\lambda \in \Sigma_{a, \varphi}=\{\lambda \in \mathbb{C}$ : $|\arg (\lambda-a)| \leq \varphi\}, \varphi<\pi$.

Prova: Vamos começar localizando a imagem numérica de $A$. Primeiramente note que

$$
W(A)=\{\langle A x, x\rangle: x \in D(A),\|x\|=1\} \subset(-\infty, a] .
$$

Note que $A-a=A^{*}-a$ são dissipativos e portanto, do Teorema 1.1.2, $\rho(A-a) \supset(0, \infty)$. Do Teorema (1.1.3) temos que $\mathbb{C} \backslash(-\infty, a] \subset \rho(A)$ e que

$$
\left\|(\lambda-A)^{-1}\right\| \leq \frac{1}{d(\lambda, W(A))} \leq \frac{1}{d(\lambda,(-\infty, a])} .
$$

Além disso, se $\lambda \in \Sigma_{a, \varphi}$ temos que

$$
\frac{1}{d(\lambda,(-\infty, a])} \leq \frac{1}{\sin \varphi} \frac{1}{|\lambda-a|}
$$

e o resultado segue. $\square$

\subsection{Continuidade do Espectro}

O objetivo desta seção é apresentar o Teorema 1.2.1 que, sob certas hipóteses, nos dará a convergência de auto-valores e auto-vetores de sequência de operadores lineares. Nesta seção, para facilitar a leitura, reescrevemos novamente as noções de convergência e compacidade que faremos uso no decorrer do texto.

Seja $X_{\epsilon}$ uma família de espaços de Banach, $\epsilon \in[0,1]$, e assuma que existe uma família de operadores lineares contínuos $E_{\epsilon}: X \rightarrow X_{\epsilon}$ com a propriedade

$$
\left\|E_{\epsilon} u\right\|_{X_{\epsilon}} \stackrel{\epsilon \rightarrow 0}{\rightarrow}\|u\|_{X}, \text { para todo } u \in X
$$

Definição 1.2.1. Dizemos que uma sequência $\left\{u_{\epsilon}\right\}_{\epsilon \in(0,1]}$, com $u_{\epsilon} \in X_{\epsilon}$ para todo $\epsilon \in[0,1]$, E-converge para u se $\left\|u_{\epsilon}-E_{\epsilon} u\right\|_{X_{\epsilon}} \stackrel{\epsilon \rightarrow 0}{\longrightarrow} 0$. Escrevemos $u_{\epsilon} \stackrel{E}{\longrightarrow}$ u para dizer que a sequência $\left\{u_{\epsilon}\right\}_{\epsilon \in[0,1]}$ E-converge para u quando $\epsilon$ tende a zero. 
Com esta noção de convergência apresentamos a definição de sequência $E$-relativamente compacta.

Definição 1.2.2. Uma sequência $\left\{u_{n}\right\}_{N \in \mathbb{N}}$, com $u_{n} \in X_{\epsilon_{n}}$ e $\epsilon_{n} \rightarrow 0$, é dita E-relativamente compacta se, para cada subsequência $\left\{u_{n^{\prime}}\right\}$ de $\left\{u_{n}\right\}$, existe uma subsequência $\left\{u_{n^{\prime \prime}}\right\}$ de $\left\{u_{n^{\prime}}\right\}$ e um elemento $u \in X$ tal que $u_{\epsilon_{n^{\prime \prime}}} \stackrel{E}{\longrightarrow} u$. A familia $\left\{u_{\epsilon}\right\}_{\epsilon \in(0,1]}$ é dita E-relativamente compacta se cada sequência $\left\{u_{\epsilon_{n}}\right\}, \epsilon_{n} \rightarrow 0$, é E-relativamente compacta.

Definição 1.2.3. Dizemos que a família de operadores $\left\{B_{\epsilon} \in \mathcal{L}\left(X_{\epsilon}\right)\right\}_{\epsilon \in[0,1]}$ EE-converge para $B_{0}$ quando $\epsilon \rightarrow 0$, se $B_{\epsilon} u_{\epsilon} \stackrel{E}{\longrightarrow} B_{0} u$ sempre que $u_{\epsilon} \stackrel{E}{\longrightarrow} u \in X$. Escreveremos $B_{\epsilon} \stackrel{E E}{\longrightarrow} B_{0}$ quando $\epsilon \rightarrow 0$ para denotar que $\left\{B_{\epsilon} \in \mathcal{L}\left(X_{\epsilon}\right)\right\}_{\epsilon \in[0,1]}$ EE-converge para $B_{0}$.

Definição 1.2.4. Dizemos que uma família de operadores compactos $\left\{B_{\epsilon} \in \mathcal{K}\left(X_{\epsilon}\right): \epsilon \in\right.$ $[0,1]\}$ converge compactamente para $B_{0}$ se, para qualquer familia $\left\{u_{\epsilon}\right\}$ com $u_{\epsilon} \in X_{\epsilon},\left\|u_{\epsilon}\right\|_{X_{\epsilon}}=$ $1, \epsilon \in(0,1]$, a família $\left\{B_{\epsilon} u_{\epsilon}\right\}$ é E-relativamente compacta e, além disso, $B_{\epsilon} \stackrel{E E}{\longrightarrow} B_{0}$. Escreveremos $B_{\epsilon} \stackrel{C C}{\longrightarrow} B_{0}$ quando $\epsilon \rightarrow 0$ para denotar que $\left\{B_{\epsilon} \in \mathcal{K}\left(X_{\epsilon}\right)\right\}_{\epsilon \in[0,1]}$ converge compactamente para $B_{0}$.

Definição 1.2.5. Seja $X$ um espaço de Banach sobre $\mathbb{C}$ e $A: D(A) \subset X \rightarrow X$ um operador linear fechado. O conjunto resolvente de $A$ é o subconjunto $\rho(A)$ de todos os $\lambda$ em $\mathbb{C}$ tais que $\lambda-A$ é bijetor. $O$ espectro de $A$ é definido como $\mathbb{C}-\rho(A)$.

Definição 1.2.6. Seja $X$ um espaço de Banach sobre $\mathcal{K}$ e $A: D(A) \subset X \rightarrow X$ um operador fechado e com resolvente não vazio. Dizemos que $A$ tem resolvente compacto se para algum $\lambda_{0} \in \rho(A)$ temos que $\left(\lambda_{0}-A\right)^{-1} \in \mathcal{K}(X)$.

Lema 1.2.1. Seja $\left\{B_{\epsilon} \in \mathcal{K}\left(X_{\epsilon}\right)\right\}_{\epsilon \in[0,1]}$ tal que $B_{\epsilon} \stackrel{C C}{\longrightarrow} B_{0}$ quando $\epsilon \rightarrow 0$. Então,

i) existe $\epsilon_{0} \in(0,1]$ tal que $\sup _{\epsilon \in\left(0, \epsilon_{0}\right]}\left\|B_{\epsilon}\right\|_{\mathcal{L}\left(X_{\epsilon}\right)}<\infty$.

ii) se $\mathcal{N}\left(I+B_{0}\right)=\{0\}$, existe $\epsilon_{0}>0$ e $M>0$ tal que

$$
\left\|\left(I+B_{\epsilon}\right)^{-1}\right\|_{\mathcal{L}\left(X_{\epsilon}\right)} \leqslant M, \quad \forall \epsilon \in\left[0, \epsilon_{0}\right] .
$$

Prova: $i)$ Se $\left\{\left\|B_{\epsilon}\right\|_{\mathcal{L}\left(X_{\epsilon}\right)}: \epsilon \in\left(0, \epsilon_{0}\right]\right\}$ não é limitada para qualquer escolha de $\epsilon_{0} \in(0,1]$, existe sequência $\left\{\epsilon_{n}\right\}$ em $(0,1]$ com $\epsilon_{n} \stackrel{n \rightarrow \infty}{\longrightarrow} 0$ e $u_{\epsilon_{n}} \in X_{\epsilon_{n}}$ com $\left\|u_{\epsilon_{n}}\right\|_{X_{\epsilon_{n}}}=1$ tal que $\left\|B_{\epsilon_{n}} u_{\epsilon_{n}}\right\| \rightarrow+\infty$ e isto está em contradição com a convergência compacta de $B_{\epsilon}$ para $B_{0}$.

ii) Como $B_{\epsilon} \in \mathcal{K}\left(X_{\epsilon}\right)$ para cada $\epsilon \in[0,1]$, segue da Alternativa de Fredholm que a estimativa (1.6) é equivalente a

$$
\left\|\left(I+B_{\epsilon}\right) u_{\epsilon}\right\|_{X_{\epsilon}} \geqslant \frac{1}{M}, \quad \forall \epsilon \in\left[0, \epsilon_{0}\right] \text { e } \forall u_{\epsilon} \in X_{\epsilon} \operatorname{com}\left\|u_{\epsilon}\right\|=1 .
$$


Suponha que isto é falso; isto é, suponha que existe uma sequência $\left\{u_{n}\right\}$, com $u_{n} \in X_{\epsilon_{n}}$, $\left\|u_{n}\right\|=1$ e $\epsilon_{n} \rightarrow 0$ tal que $\left\|\left(I+B_{\epsilon_{n}}\right) u_{n}\right\| \rightarrow 0$. Como $\left\{B_{\epsilon_{n}} u_{n}\right\}$ tem uma subsequência $E$-convergente, que novamente denotamos por $\left\{B_{\epsilon_{n}} u_{n}\right\}$, para $u,\|u\|=1$, segue que $u_{n}+$ $B_{\epsilon_{n}} u_{n} \stackrel{E}{\longrightarrow} 0$ e $u_{n} \stackrel{E}{\longrightarrow}-u$. Isto implica que $\left(I+B_{0}\right) u=0$ e isto está em contradição com a hipótese $N\left(I+B_{0}\right)=\{0\} \cdot \square$

Em geral, os operadores $B_{\epsilon}$ serão inversas de certos operadores diferenciais $A_{\epsilon}$. Assim, considere a família de operadores $\left\{A_{\epsilon}: D\left(A_{\epsilon}\right) \subset X_{\epsilon} \rightarrow X_{\epsilon}, \epsilon \in[0,1]\right\}$ e suponha que, para todo $\epsilon \in[0,1]$,

$$
A_{\epsilon} \text { é fechado, tem resolvente compacto } 0 \in \rho\left(A_{\epsilon}\right) \text {, e } A_{\epsilon}^{-1} \stackrel{C C}{\longrightarrow} A_{0}^{-1} \text {. }
$$

Lema 1.2.2. Suponha que a familia de operadores $\left\{A_{\epsilon}: D\left(A_{\epsilon}\right) \subset X_{\epsilon} \rightarrow X_{\epsilon}, \epsilon \in[0,1]\right\}$ satisfaz (1.7). Então, para cada $\lambda \in \rho\left(A_{0}\right)$, existe $\epsilon_{\lambda}>0$ tal que $\lambda \in \rho\left(A_{\epsilon}\right)$ para todo $\epsilon \in\left[0, \epsilon_{\lambda}\right]$ e existe uma constante $M_{\lambda}>0$ tal que

$$
\left\|\left(\lambda-A_{\epsilon}\right)^{-1}\right\| \leqslant M_{\lambda}, \quad \forall \epsilon \in\left[0, \epsilon_{\lambda}\right] .
$$

Além disso, $\left(\lambda-A_{\epsilon}\right)^{-1} \stackrel{C C}{\longrightarrow}\left(\lambda-A_{0}\right)^{-1}$ quando $\epsilon \rightarrow 0$.

Prova: De (1.7) e do fato que $\lambda \in \rho\left(A_{0}\right)$ é fácil ver que $\left(\lambda-A_{0}\right)^{-1}=-A_{0}^{-1}\left(I-\lambda A_{0}^{-1}\right)^{-1}$. Como $A_{\epsilon}^{-1} \stackrel{C C}{\longrightarrow} A_{0}^{-1}$, aplicando o Lema $\left.1.2 .1 \mathrm{i}\right)$ e ii), obtemos que o operador $-A_{\epsilon}^{-1}(I-$ $\left.\lambda A_{\epsilon}^{-1}\right)^{-1}$ está bem definido e é limitado. Cálculos simples mostram que $-A_{\epsilon}^{-1}\left(I-\lambda A_{\epsilon}^{-1}\right)^{-1}=$ $\left(\lambda-A_{\epsilon}\right)^{-1}$. Logo $\lambda \in \rho\left(A_{\epsilon}\right)$ e obtemos $(1.8)$.

Para provar a convergência compacta de $\left(\lambda-A_{\epsilon}\right)^{-1}$ para $\left(\lambda-A_{0}\right)^{-1}$ procedemos da seguinte maneira: Como $A_{\epsilon}^{-1}$ converge compactamente para $A_{0}^{-1}$ e como $\left\{\left(I-\lambda A_{\epsilon}^{-1}\right): 0 \leqslant \epsilon \leqslant \epsilon_{\lambda}\right\}$ é limitado, concluímos que

- Se $\left\|u_{\epsilon}\right\|_{X_{\epsilon}}=1$ então $\left(\lambda-A_{\epsilon}\right)^{-1} u_{\epsilon}=-A_{\epsilon}^{-1} w_{\epsilon} \operatorname{com} w_{\epsilon}=\left(I-\lambda A_{\epsilon}^{-1}\right)^{-1} u_{\epsilon}$ que é uniformemente limitado em $\epsilon$. Logo $\left(\lambda-A_{\epsilon}\right)^{-1} u_{\epsilon}$ tem uma subsequência $E$-convergente.

- Se $u_{\epsilon} \stackrel{E}{\longrightarrow} u$ então $A_{\epsilon}^{-1} u_{\epsilon} \stackrel{E}{\longrightarrow} A_{0}^{-1} u$. Agora, para qualquer subseqfuência de $\{(\lambda-$ $\left.\left.A_{\epsilon}\right)^{-1} u_{\epsilon}\right\}$ existe uma subsequência (que novamente denotamos por $\left\{\left(\lambda-A_{\epsilon}\right)^{-1} u_{\epsilon}\right\}$ ) e $y \in X$ tal que,

$$
\left(\lambda-A_{\epsilon}\right)^{-1} u_{\epsilon}=-\left(I-\lambda A_{\epsilon}^{-1}\right)^{-1} A_{\epsilon}^{-1} u_{\epsilon}=-A_{\epsilon}^{-1}\left(I-\lambda A_{\epsilon}^{-1}\right)^{-1} u_{\epsilon}=z_{\epsilon} \stackrel{E}{\longrightarrow} y .
$$

Logo,

$$
A_{0}^{-1} u \stackrel{E}{\longleftarrow} A_{\epsilon}^{-1} u_{\epsilon}=-\left(I-\lambda A_{\epsilon}^{-1}\right) z_{\epsilon} \stackrel{E}{\longrightarrow}-\left(I-\lambda A_{0}^{-1}\right) y
$$


e isto implica que $y=\left(\lambda-A_{0}\right)^{-1} u$. Em particular, $y$ é independente da subsequência tomada. Isto implica que a sequência inteira $\left(\lambda-A_{\epsilon}\right)^{-1} u_{\epsilon} E$-converge para $y=(\lambda-$ $\left.A_{0}\right)^{-1} u$ quando $\epsilon \rightarrow 0$. Portanto, $\left(\lambda-A_{\epsilon}\right)^{-1} \stackrel{E E}{\longrightarrow}\left(\lambda-A_{0}\right)^{-1}$ quando $\epsilon \rightarrow 0$.

Disto segue a convergência compacta $\left(\lambda-A_{\epsilon}\right)^{-1} \stackrel{C C}{\longrightarrow}\left(\lambda-A_{0}\right)^{-1}$ quando $\epsilon \rightarrow 0$ e o resultado está provado. $\square$

Lema 1.2.3. Suponha que a família de operadores $\left\{A_{\epsilon}: D\left(A_{\epsilon}\right) \subset X_{\epsilon} \rightarrow X_{\epsilon}, \epsilon \in[0,1]\right\}$ satisfaz (1.7). Se $\Sigma$ é um subconjunto compacto de $\rho\left(A_{0}\right)$, existe $\epsilon_{\Sigma}>0$ tal que $\Sigma \subset \rho\left(A_{\epsilon}\right)$ para todo $\epsilon \leqslant \epsilon_{\Sigma} e$

$$
\sup _{\epsilon \in\left[0, \epsilon_{\Sigma}\right]} \sup _{\lambda \in \Sigma}\left\|\left(\lambda-A_{\epsilon}\right)^{-1}\right\|_{\mathcal{L}\left(X_{\epsilon}\right)}<\infty
$$

Além disso, se existe $\epsilon_{0} \in(0,1]$ tal que $\sup _{\epsilon \in\left[0, \epsilon_{0}\right]}\left\|E_{\epsilon}\right\|_{\mathcal{L}\left(X_{0}, X_{\epsilon}\right)}<\infty$ então, para cada $u \in X$ temos que

$$
\sup _{\lambda \in \Sigma}\left\|\left(\lambda-A_{\epsilon}\right)^{-1} E_{\epsilon} u-E_{\epsilon}\left(\lambda-A_{0}\right)^{-1} u\right\|_{X_{\epsilon}} \stackrel{\epsilon \rightarrow 0}{\longrightarrow} 0 .
$$

Prova: Primeiramente mostremos que existe $\hat{\epsilon}_{\Sigma}>$ tal que $\Sigma \subset \rho\left(A_{\epsilon}\right)$ para todo $\epsilon \in\left[0, \hat{\epsilon}_{\Sigma}\right)$. Se este não fosse o caso, existiriam seqüências $\epsilon_{n} \rightarrow 0, \lambda_{n} \in \Sigma$ (que podemos supor convergente para um $\lambda \in \Sigma)$ e $u_{\epsilon_{n}} \in X_{\epsilon_{n}},\left\|u_{\epsilon_{n}}\right\|=1$ tais que $A_{\epsilon_{n}} u_{\epsilon_{n}}-\lambda_{n} u_{\epsilon_{n}}=0$ ou, equivalentemente, $\lambda_{n}\left(A_{\epsilon_{n}}\right)^{-1} u_{\epsilon_{n}}=u_{\epsilon_{n}}$. Da convergência compacta $\left\{u_{\epsilon_{n}}\right\}$ tem uma subsequência $E$-convergente para $u \in X,\|u\|_{X}=1$ e $A_{0} u=\lambda u$ o que está em contradição com $\sigma\left(A_{0}\right) \cap \Sigma=\emptyset$.

Mostremos que existe $\epsilon_{\Sigma} \in\left(0, \hat{\epsilon}_{\Sigma}\right)$ tal que (1.9) vale. Para isto, é suficiente provar que existe $\epsilon_{\Sigma} \in(0,1]$ tal que

$$
\left\{\left\|\left(I-\lambda A_{\epsilon}^{-1}\right)^{-1}\right\|_{\mathcal{L}\left(X_{\epsilon}\right)}: \epsilon \in\left[0, \epsilon_{\Sigma}\right] \text { e } \lambda \in \Sigma\right\} \text { é limitado. }
$$

Se este não fosse o caso, existiria uma sequência $\left\{\lambda_{n}\right\}$ em $\Sigma$ (que podemos supor convergente para um certo $\tilde{\lambda} \in \Sigma)$ e uma sequência $\left\{\epsilon_{n}\right\}$ em $(0,1] \operatorname{com} \epsilon_{n} \stackrel{n \rightarrow \infty}{\longrightarrow} 0$ tal que

$$
\left\|\left(I-\lambda_{n}\left(A_{\epsilon_{n}}\right)^{-1}\right)^{-1}\right\|_{\mathcal{L}\left(X_{\epsilon_{n}}\right)} \stackrel{n \rightarrow \infty}{\longrightarrow} \infty
$$

Do Lema 1.2.1 obtemos uma contradição, já que $-\lambda_{n}\left(A_{\epsilon_{n}}\right)^{-1} \stackrel{C C}{\longrightarrow}-\tilde{\lambda}\left(A_{0}\right)^{-1}$ quando $n \rightarrow \infty$.

Também provamos (1.10) por contradição. Suponha que existem seqüências $\epsilon_{n} \rightarrow 0, \Sigma \ni \lambda_{n} \rightarrow \bar{\lambda} \in \Sigma$ e $\eta>0$ tal que

$$
\left\|\left(\lambda_{n}-A_{\epsilon_{n}}\right)^{-1} E_{\epsilon_{n}} u-E_{\epsilon_{n}}\left(\lambda_{n}-A_{0}\right)^{-1} u\right\|_{X_{\epsilon_{n}}} \geqslant \eta .
$$

Usando a identidade do resolvente, temos que

$$
\left(\lambda_{n}-A_{\epsilon_{n}}\right)^{-1} E_{\epsilon_{n}} u-\left(\bar{\lambda}-A_{\epsilon_{n}}\right)^{-1} E_{\epsilon_{n}} u=\left(\bar{\lambda}-\lambda_{n}\right)\left(\lambda_{n}-A_{\epsilon_{n}}\right)^{-1}\left(\bar{\lambda}-A_{\epsilon_{n}}\right)^{-1} E_{\epsilon_{n}} u .
$$


Disto e de (1.9) segue que

$$
\left\|\left(\lambda_{n}-A_{\epsilon_{n}}\right)^{-1} E_{\epsilon_{n}} u-\left(\bar{\lambda}-A_{\epsilon_{n}}\right)^{-1} E_{\epsilon_{n}} u\right\|_{X_{\epsilon_{n}}} \stackrel{n \rightarrow \infty}{\longrightarrow} 0 .
$$

Do Lema 1.2.2 temos que

$$
\left\|\left(\bar{\lambda}-A_{\epsilon_{n}}\right)^{-1} E_{\epsilon_{n}} u-E_{\epsilon_{n}}\left(\bar{\lambda}-A_{0}\right)^{-1} u\right\|_{X_{\epsilon_{n}}} \stackrel{n \rightarrow \infty}{\longrightarrow} 0 .
$$

Finalmente, da continuidade do resolvente obtemos

$$
\left\|\left(\lambda_{n}-A_{0}\right)^{-1} u-\left(\bar{\lambda}-A_{0}\right)^{-1} u\right\|_{X} \rightarrow 0 \text { as } n \rightarrow \infty .
$$

Agora, (1.12), (1.13) e (1.14) estão em contradição com (1.11) e o resultado está provado.

Para cada $\delta>0$ e $\lambda_{0} \in \mathbb{C}$ defina $S_{\delta}\left(\lambda_{0}\right):=\left\{\mu \in \mathbb{C}:\left|\lambda-\lambda_{0}\right|=\delta\right\}$.

A um ponto isolado $\lambda \in \sigma\left(A_{0}\right)$ associamos o seu auto-espaço generalizado $W\left(\lambda, A_{0}\right)=$ $Q\left(\lambda, A_{0}\right) X$ onde

$$
Q\left(\lambda, A_{0}\right)=\frac{1}{2 \pi i} \int_{|\xi-\lambda|=\delta}\left(\xi I-A_{0}\right)^{-1} d \xi
$$

e $\delta$ é escolhido de forma que não haja nenhum outro ponto de $\sigma\left(A_{0}\right)$ no disco $\bar{B}_{\delta}^{\mathbb{C}}(\lambda)=\{\xi \in$ $\mathbb{C}:|\xi-\lambda| \leqslant \delta\}$. Segue do Lema 1.2 .3 que existe $\epsilon_{S_{\delta}(\lambda)}$ tal que $\rho\left(A_{\epsilon}\right) \supset S_{\delta}(\lambda)$ para todo $\epsilon \leqslant \epsilon_{S_{\delta}(\lambda)}$. Seja $W\left(\lambda, A_{\epsilon}\right):=Q\left(\lambda, A_{\epsilon}\right) X_{\epsilon}$ onde

$$
Q\left(\lambda, A_{\epsilon}\right)=\frac{1}{2 \pi i} \int_{|\xi-\lambda|=\delta}\left(\xi I-A_{\epsilon}\right)^{-1} d \xi .
$$

Lema 1.2.4. Seja $X$ um espaço de Banach. Se $M, N$ são subespaços de $X$ com $\operatorname{dim}(M)$ $>\operatorname{dim}(N)$, então existe $u \in M,\|u\|=1$ tal que $\operatorname{dist}(u, N)=1$.

O resultado a seguir diz que o espectro de $A_{\epsilon}$, para $\epsilon$ pequeno, se aproxima do espectro de $A_{0}$. Sabe-se que o espectro de $A_{\epsilon}$ ou $A_{0}$ contém apenas auto-valores isolados de multiplicidade finita.

Teorema 1.2.1. Seja $\left\{A_{\epsilon}: D\left(A_{\epsilon}\right) \subset X_{\epsilon} \rightarrow X_{\epsilon}, \epsilon \in[0,1]\right\}$ uma família de operadores tal que (1.7) está satisfeita. Então, valem as seguintes afirmativas:

(i) Se $\lambda_{0} \in \sigma\left(A_{0}\right)$, existe seqüência $\left\{\epsilon_{n}\right\}$ em $(0,1]$ com $\epsilon_{n} \stackrel{n \rightarrow \infty}{\longrightarrow} 0$ e sequência $\left\{\lambda_{n}\right\}$ em $\mathbb{C}$ $\operatorname{com} \lambda_{n} \in \sigma\left(A_{\epsilon_{n}}\right)$, para $n=1,2,3 \cdots$, e $\lambda_{n} \stackrel{n \rightarrow \infty}{\longrightarrow} \lambda_{0}$.

(ii) Se $\left\{\epsilon_{n}\right\}$ é uma sequência em $(0,1]$ com $\epsilon_{n} \stackrel{n \rightarrow \infty}{\longrightarrow} 0, e\left\{\lambda_{n}\right\}$ é uma sequência em $\mathbb{C}$ com $\lambda_{n} \in \sigma\left(A_{\epsilon_{n}}\right), n \in \mathbb{N}$ e $\lambda_{n} \stackrel{n \rightarrow \infty}{\longrightarrow} \lambda_{0}$, então $\lambda_{0} \in \sigma\left(A_{0}\right)$. 
Além disso, se existe $\epsilon_{0} \in(0,1]$ tal que $\sup _{\epsilon \in\left[0, \epsilon_{0}\right]}\left\|E_{\epsilon}\right\|_{\mathcal{L}\left(X_{0}, X_{\epsilon}\right)}<\infty$, valem as seguintes afirmativas:

(iii) Existe $\epsilon_{1} \in\left(0, \epsilon_{0}\right)$ tal que $\operatorname{dim} W\left(\lambda, A_{\epsilon}\right)=\operatorname{dim} W\left(\lambda_{0}, A_{0}\right)$ para todo $0 \leqslant \epsilon \leqslant \epsilon_{1}$.

(iv) Se $u \in W\left(\lambda_{0}, A_{0}\right)$, então existe uma sequência $\left\{\epsilon_{n}\right\}$ em $(0,1]$ com $\epsilon_{n} \stackrel{n \rightarrow \infty}{\longrightarrow} 0, u_{\epsilon_{n}} \in$ $W\left(\lambda_{0}, A_{\epsilon_{n}}\right)$ e tal que $u_{\epsilon_{n}} \stackrel{E}{\longrightarrow}$ u quando $n \rightarrow \infty$.

(v) Se $\left\{\epsilon_{n}\right\}$ é uma sequência em $(0,1]$ com $\epsilon_{n} \stackrel{n \rightarrow \infty}{\longrightarrow} 0$, e $\left\{u_{n}\right\}$ é uma sequência com $u_{n} \in$ $W\left(\lambda, A_{\epsilon_{n}}\right),\left\|u_{n}\right\|_{X_{\epsilon_{n}}}=1$, então $\left\{u_{n}\right\}$ tem uma subsequência E-convergente para um vetor $u \in W\left(\lambda_{0}, A_{0}\right)$.

Prova. (i) Seja $\lambda_{0} \in \sigma\left(A_{0}\right)$ e $\delta>0$ tal que $\bar{B}_{\delta}^{\mathbb{C}}\left(\lambda_{0}\right) \cap \sigma\left(A_{0}\right)=\left\{\lambda_{0}\right\}$. Do Lema 1.2.3, existe $\epsilon_{0}>0$ tal que $\left\{\left\|\left(\lambda-A_{\epsilon}\right)^{-1}\right\|_{\mathcal{L}\left(X_{\epsilon}\right)}: \epsilon \in\left[0, \epsilon_{0}\right]\right.$ e $\left.\lambda \in S_{\delta}\left(\lambda_{0}\right)\right\}$ é limitado.

Suponha agora que, existe $\delta>0$ e seqüência $\epsilon_{n} \stackrel{n \rightarrow \infty}{\longrightarrow} 0$ tal que, $\bar{B}_{\delta}\left(\lambda_{0}\right) \subset \rho\left(A_{\epsilon_{n}}\right)$ para todo $n \in \mathbb{N}$. Como $\bar{B}_{\delta}\left(\lambda_{0}\right) \ni \lambda \mapsto\left(\lambda-A_{\epsilon_{n}}\right)^{-1} \in \mathcal{L}(X)$ é analítica para cada $n \in \mathbb{N}$, da prova do Lema 1.2 .3 e do Teorema do Máximo Módulo temos que

$$
\left\|\left(I-\lambda_{0} A_{\epsilon_{n}}^{-1}\right)^{-1}\right\|_{\mathcal{L}\left(X_{\epsilon_{n}}\right)} \leqslant \sup _{\substack{\left|\lambda-\lambda_{0}\right|=\delta \\ n \in \mathbb{N}}}\left\|\left(I-\lambda A_{\epsilon_{n}}^{-1}\right)^{-1}\right\|_{\mathcal{L}\left(X_{\epsilon_{n}}\right)}<\infty .
$$

Portanto, se $u_{\epsilon} \stackrel{E}{\longrightarrow} u$, segue que

$$
\left\|\left(\lambda_{0} A_{0}^{-1}-I\right) u\right\|_{X}=\lim _{\epsilon \rightarrow 0}\left\|\left(\lambda_{0} A_{\epsilon}^{-1}-I\right) u_{\epsilon}\right\|_{X_{\epsilon}} \geqslant c\|u\|_{X}
$$

para algum $c>0$ e, consequentemente, $\lambda_{0} \in \rho\left(A_{0}\right)$. Isto contradiz a escolha de $\lambda_{0}$ e prova que, para cada $\delta>0, \bar{B}_{\delta}\left(\lambda_{0}\right)$ contém algum ponto de $\sigma\left(A_{\epsilon}\right)$, para todo $\epsilon$ suficientemente pequeno.

(ii) Sejam $\left\{\epsilon_{n}\right\}$ uma sequência em $(0,1] \operatorname{com} \epsilon_{n} \stackrel{n \rightarrow \infty}{\longrightarrow} 0,\left\{\lambda_{n}\right\}$ uma sequência em $\mathbb{C}$ com $\lambda_{n} \in \sigma\left(A_{\epsilon_{n}}\right)$ tal que $\lambda_{n} \stackrel{n \rightarrow \infty}{\longrightarrow} \lambda$ e $\left\{u_{n}\right\}$ uma sequência com $u_{n} \in X_{\epsilon_{n}},\left(I-\lambda_{n}\left(A_{\epsilon_{n}}\right)^{-1}\right) u_{n}=0$ e $\left\|u_{n}\right\|=1$. Então

$$
\left\|\left(I-\lambda\left(A_{\epsilon_{n}}\right)^{-1}\right) u_{n}\right\|_{X_{\epsilon_{n}}}=\left\|\left(I-\lambda_{n}\left(A_{\epsilon_{n}}\right)^{-1}\right) u_{n}-\left(\lambda-\lambda_{n}\right)\left(A_{\epsilon_{n}}\right)^{-1} u_{n}\right\|_{X_{\epsilon_{n}}} \rightarrow 0
$$

quando $n \rightarrow \infty$. Uma vez que $\left\|u_{n}\right\|=1$ temos, tomando subsequências se necessário, $\lambda\left(A_{\epsilon_{n}}\right)^{-1} u_{n} \stackrel{E}{\longrightarrow} u$ e $u_{n} \stackrel{E}{\longrightarrow} u$ com $\|u\|=1$. Portanto $u-\lambda A_{0}^{-1} u=0, u \neq 0$ e $\lambda \in \sigma\left(A_{0}\right)$.

(iii) Como $\left(\lambda-A_{\epsilon}\right)^{-1} \stackrel{E E}{\longrightarrow}\left(\lambda-A_{0}\right)^{-1}$ uniformemente para $\lambda \in S_{\delta}\left(\lambda_{0}\right)$ (veja (1.10) no Lema 1.2.3) segue que $Q_{\epsilon}\left(\lambda_{0}\right) \stackrel{E E}{\longrightarrow} Q\left(\lambda_{0}\right)$ quando $\epsilon \rightarrow 0$. 
Se $v_{1}, \cdots, v_{k}$ é uma base para $W\left(\lambda_{0}, A_{0}\right)=Q_{0}\left(\lambda_{0}\right) x$, é fácil ver que, para $\epsilon$ suficientemente pequeno,

$$
\left\{Q_{\epsilon}\left(\lambda_{0}\right) E_{\epsilon} v_{1}, \cdots, Q_{\epsilon}\left(\lambda_{0}\right) E_{\epsilon} v_{k}\right\}
$$

é um conjunto linearmente independente em $Q_{\epsilon}\left(\lambda_{0}\right) X_{\epsilon}$. Disto segue que $\operatorname{dim}\left(Q_{\epsilon}\left(\lambda_{0}\right)\left(X_{\epsilon}\right)\right) \geqslant$ $\operatorname{dim}\left(Q\left(\lambda_{0}\right)(X)\right)$.

Provamos a igualdade supondo que $Q_{\epsilon}\left(\lambda_{0}\right) \stackrel{C C}{\longrightarrow} Q\left(\lambda_{0}\right)$. Suponha, por redução ao absurdo que, para alguma sequência $\epsilon_{n} \stackrel{n \rightarrow \infty}{\longrightarrow} 0$,

$$
\operatorname{dim}\left(Q_{\epsilon_{n}}\left(\lambda_{0}\right)\left(X_{\epsilon_{n}}\right)\right)>\operatorname{dim}\left(Q\left(\lambda_{0}\right)(X)\right)
$$

Do Lema 1.2.4 segue que, para cada $n \in \mathbb{N}$, existe $u_{n} \in W\left(\lambda_{0}, A_{\epsilon_{n}}\right)$ com $\left\|u_{n}\right\|=1$ tal que $\operatorname{dist}\left(u_{n}, E_{\epsilon_{n}} W\left(\lambda_{0}, A_{0}\right)\right)=1$. Da convergência compacta podemos supor que $Q_{\epsilon_{n}}\left(\lambda_{0}\right) u_{n}=$ $u_{n} \stackrel{E}{\longrightarrow} Q_{0}\left(\lambda_{0}\right) u_{0}=u_{0}$ e temos um absurdo, já que

$$
1 \leqslant\left\|u_{n}-E_{\epsilon_{n}} Q_{0}\left(\lambda_{0}\right) u\right\|_{X_{\epsilon_{n}}}=\left\|Q_{\epsilon_{n}}\left(\lambda_{0}\right) u_{n}-E_{\epsilon_{n}} Q_{0}\left(\lambda_{0}\right) u\right\|_{X_{\epsilon_{n}}} \rightarrow 0
$$

Assim precisamos apenas provar a convergência compacta $Q_{\epsilon}\left(\lambda_{0}\right) \stackrel{C C}{\longrightarrow} Q\left(\lambda_{0}\right)$ quando $\epsilon \rightarrow 0$ e isto segue de $Q_{\epsilon}\left(\lambda_{0}\right) \stackrel{E E}{\longrightarrow} Q\left(\lambda_{0}\right)$, da convergência compacta $A_{\epsilon}^{-1} \stackrel{C C}{\longrightarrow} A_{0}^{-1}$ quando $\epsilon \rightarrow 0$, da limitação uniforme de $\left\|\left(\zeta A_{\epsilon}^{-1}-I\right)^{-1}\right\|$ para $\zeta \in S_{\delta}\left(\lambda_{0}\right)$ e $\epsilon \in\left[0, \epsilon_{0}\right]$, dada na prova do Lema 1.2.3, e da fórmula

$$
Q_{\epsilon}\left(\lambda_{0}\right)=\frac{1}{2 \pi i} \int_{\left|\zeta-\lambda_{0}\right|=\delta}\left(\zeta I-A_{\epsilon}\right)^{-1} d \zeta=A_{\epsilon}^{-1} \frac{1}{2 \pi i} \int_{\left|\zeta-\lambda_{0}\right|=\delta}\left(\zeta A_{\epsilon}^{-1}-I\right)^{-1} d \zeta .
$$

(iv) Segue tomando $u_{\epsilon}=Q_{\epsilon}\left(\lambda_{0}\right) E_{\epsilon} u$.

$(v)$ Segue da convergência compacta de $Q_{\epsilon}$ para $Q_{0}$ provada em $(i i i) \cdot \square$

\subsection{Potências Fracionárias}

O objetivo desta seção é apresentar as noções de operadores de tipo positivo, suas potências fracionárias e os principais resultados sobre estes, utilizados ao longo do texto. Utilizamos os teoremas desta seção para se obter uma taxa de $E$-convergência dos semigrupos lineares gerados pelos operadores $-L_{\epsilon}$, com $L_{\epsilon}$ definido na introdução.

Seja $X$ um espaço de Banach. Um operador linear $A$ em $X$ é dito de tipo positivo com constante $M \geq 1$, se é fechado, densamente definido, $\mathbb{R}^{+} \subset \rho(-A) \mathrm{e}$

$$
(1+s)\left\|(s+A)^{-1}\right\|_{\mathcal{L}(X)} \leq M, \quad s \in \mathbb{R}^{+}
$$


para todo $A \in \mathcal{P}(X)$ e $\alpha \in \mathbb{C}, \operatorname{Re} \alpha<0$, definimos

$$
A^{\alpha}:=\frac{1}{2 \pi i} \int_{\Gamma}(-\lambda)^{\alpha}(\lambda+A)^{-1} d \lambda=\frac{1}{2 \pi i} \int_{-\Gamma} \lambda^{\alpha}(\lambda-A)^{-1} d \lambda
$$

onde $\Gamma$ é qualquer curva simples em $\Sigma_{M} \backslash \mathbb{R}^{+}$suave por partes indo de $\infty \mathrm{e}^{-i \nu}$ até $\infty \mathrm{e}^{i \nu}$ para $\operatorname{algum} \nu \in(0, \arcsin 1 /(2 M)]$. É claro que $-\Gamma:=\{\lambda \in \mathbb{C}:-\lambda \in \Gamma\}$. Segue de (1.15) e (1.16) e do Teorema de Cauchy que $A^{\alpha}$ está bem definido em $\mathcal{L}(X)$ e independente da escolha de $\Gamma$.

Para operadores positivos temos o seguinte teorema de interpolação.

Teorema 1.3.1. Suponha que $A \in \mathcal{P}(X)$ e $0 \leq \alpha \leq 1$, então existe uma constante $K$ dependendo somente de $A$ (da constante $M$ de positividade), tal que $\left\|A^{\alpha} x\right\|_{X} \leq K\|A x\|_{X}^{\alpha}\|x\|_{X}^{1-\alpha}$ para $0 \leq \alpha \leq 1, x \in D(A)$.

Agora consideramos o caso em que $A$ é setorial; isto é, $\left\{\mathrm{e}^{-A t}, t \geq 0\right\}$ é semigrupo analítico.

Teorema 1.3.2. Suponha que A é setorial. Logo $\left\{\mathrm{e}^{-A t} ; t \geq 0\right\}$ é um semigrupo analítico, suponha que $\rho(A) \supset(-\infty, 0]$. Então

1. Se $t>0, \alpha \geq 0, R\left(\mathrm{e}^{-A t}\right) \subset D\left(A^{\alpha}\right) e$

$$
\left\|A^{\alpha} \mathrm{e}^{-A t}\right\|_{\mathcal{L}(X)} \leq M_{\alpha} t^{-\alpha} e^{a t}, \quad t>0
$$

onde a é o vértice do setor como no Teorema 0.0.4. Mais ainda, $\alpha \mapsto M_{\alpha}$ é contínua em $[0, \infty)$.

2. Se $\alpha>0$, temos que $t^{\alpha} A^{\alpha} \mathrm{e}^{-A t} x \rightarrow 0$ quando $t \rightarrow 0^{+}$para cada $x \in X$.

3. $\left\|\left(\mathrm{e}^{-A t}-I\right) A^{-\alpha}\right\|_{\mathcal{L}(X)} \leq M_{1-\alpha} \frac{t^{\alpha}}{\alpha}$ se $0<\alpha \leq 1,0 \leq t \leq 1$.

Definição 1.3.1. Dizemos que $A$ é do tipo $(\omega, M)$ em um espaço de Banach $X$ se $A$ é fechado, densamente definito e o resolvente de $-A$ contém um setor aberto $\{\lambda \in \mathbb{C}:|\arg (\lambda)|<$ $\pi-\omega\}$ e $\lambda(\lambda+A)^{-1}$ é uniformemente limitado em cada setor menor $\{\lambda \in \mathbb{C}:|\arg (\lambda)|<$ $\pi-\omega-\epsilon\}, \epsilon>0$ e $\left\|\lambda(\lambda+A)^{-1}\right\| \leq M$ para todo $\lambda \geq 0$.

Teorema 1.3.3. Seja A um operador de tipo $(\omega, M)$ em um espaço de Banach $X$ com $0 \in \rho(A)$ e $0<\alpha<1$. Então $A^{\alpha}$ está definido e é de tipo $(\alpha \omega, M)$. Em particupar, $A^{\alpha}$ é de tipo positivo. 


\subsection{Teoremas de Trotter-Kato}

Nesta seção enunciamos e demonstramos um teorema que caracteriza a relação entre convergência de operadores resolventes e a convergência de semigrupos lineares associados.

Denotaremos $A \in G(M, \omega)$ se $A$ é o gerador infinitesimal de um semigrupo fortemente contínuo $T(t)$ que satisfaz

$$
\|T(t)\|_{\mathcal{L}(X)} \leq M \mathrm{e}^{\beta t}, \quad \forall t \geq 0 .
$$

A seguir, enunciaremos teoremas que se encontram em [14] e que caracterizam esta relação quando o domínio dos operadores está fixado.

Teorema 1.4.1. Sejam $A, A_{n} \in G(M, \omega)$ e $e^{A_{n} t}$ o semigrupo cujo gerador infinitesimal é $A_{n}$. Então são equivalentes:

(a) Para todo $x \in X$ e $\lambda$ com $\operatorname{Re} \lambda>\omega,\left(\lambda-A_{n}\right)^{-1} x \rightarrow(\lambda-A)^{-1} x$ quando $n \rightarrow \infty$.

(b) Para todo $x \in X$ e $t \geq 0$, $\mathrm{e}^{A_{n} t} x \rightarrow \mathrm{e}^{A t} x$ quando $n \rightarrow \infty$.

Além disso, a convergência na parte (b) é uniforme para t em intervalos limitados.

Na demonstração do teorema acima pode-se notar que a condição $(a)$ pode ser enfraquecida para a convergência em apenas um ponto $\lambda \operatorname{com} \operatorname{Re}(\lambda)>\omega$. Desta forma, o resultado anterior está relacionado com o seguinte teorema.

Teorema 1.4.2. Sejam $A_{n} \in G(M, \omega)$. Se existe $\lambda_{0}$ com $\operatorname{Re}\left(\lambda_{0}\right)>\omega$ tal que

(a) Para todo $x \in X$, existe o limite $\left(\lambda-A_{n}\right)^{-1} x \rightarrow R\left(\lambda_{0}\right) x$ quando $n \rightarrow \infty$.

(b) A imagem de $R\left(\lambda_{0}\right)$ é densa em $X$,

então existe um único operador $A \in G(M, \omega)$ tal que $R\left(\lambda_{0}\right)=\left(\lambda_{0}-A\right)^{-1}$.

Como consequência dos teoremas anteriores, obtem-se o seguinte teorema.

Teorema 1.4.3 (Trotter-Kato). Sejam $A_{n} \in G(M, \omega)$ e e $e^{A_{n} t}$ o semigrupo cujo gerador infinitesimal é $A_{n}$. Se existe $\lambda_{0} \operatorname{com} \operatorname{Re}\left(\lambda_{0}\right)>\omega$ tal que

(a) Para todo $x \in X$, existe o limite $\left(\lambda-A_{n}\right)^{-1} x \rightarrow R\left(\lambda_{0}\right) x$ quando $n \rightarrow \infty$.

(b) A imagem de $R\left(\lambda_{0}\right)$ é densa em $X$,

então existe um único operador $A \in G(M, \omega)$ tal que $R\left(\lambda_{0}\right)=\left(\lambda_{0}-A\right)^{-1}$. Além disso,

$$
\mathrm{e}^{A_{n} t} x \rightarrow \mathrm{e}^{A t} x \text { quando } n \rightarrow \infty \text {. }
$$


para todo $x \in X$ e $t \geq 0$. Além disso, esta convergência é uniforme para $t$ em intervalos limitados.

Nos problemas que analisaremos a convergência, os operadores envolvidos estarão definidos em espaços distintos. Dessa forma, o teorema abaixo, para operadores setoriais, nos será mais interessante no decorrer do texto.

Teorema 1.4.4. Sejam $A: X \supset D(A) \rightarrow X, A_{n}: X_{n} \supset D\left(A_{n}\right) \rightarrow X_{n}, n \in \mathbb{N}$ operadores lineares densamente definidos tais que

$$
\left\|\left(\lambda I_{n}-A_{n}\right)^{-1}\right\| \leq \frac{M}{|\lambda-\omega|}, \forall \lambda \in \Sigma_{\omega, \varphi} \forall n \in \mathbb{N} .
$$

para algum setor $\Sigma_{\omega, \varphi}:=\{\lambda \in \mathbb{C} ;|\arg (\lambda-\omega)| \leq \varphi\}, \varphi \in\left(\frac{\pi}{2}, \pi\right)$. Então as seguintes condições são equivalentes:

1. Existe $\lambda \in \mathbb{C}$ com $\operatorname{Re}(\lambda)>\omega$ tal que

$$
\left(\lambda I_{n}-A_{n}\right)^{-1} \stackrel{E E}{\rightarrow}(\lambda I-A)^{-1}
$$

2. Existe $0<\theta<\frac{\pi}{2}$ tal que $\forall \epsilon_{0}, \mu \in(0, \infty)$, $\epsilon_{0}<\mu$ temos

$$
\sup _{t \in \Sigma\left(\theta, \epsilon_{0}, \mu\right)}\left\|e^{A_{n} t} u_{n}-E_{n} e^{A t} u_{0}\right\|_{X_{n}} \stackrel{n \rightarrow \infty}{\rightarrow} 0
$$

sempre que $u_{n} \stackrel{E}{\rightarrow} u_{0}$, onde

$$
\Sigma\left(\theta, \epsilon_{0}, \mu\right)=\left\{z \in \mathbb{C} ;|\arg (z)| \leq \theta \text { e } \epsilon_{0} \leq|t| \leq \mu\right\} .
$$

Isto é, $e^{A_{n} t} \stackrel{E E}{\rightarrow} e^{A t}$ uniformemente para $t$ em compactos $\Sigma\left(\theta, \epsilon_{0}, \mu\right)$.

Em ambos os casos, temos EE-convergência uniforme em compactos contidos em $\Sigma\left(\theta, \epsilon_{0}, \mu\right)$.

Prova: Suponha que vale (1.18). Provemos que a convergência em (1.18) é uniforme em compactos $K$ contidos em $\Sigma_{\omega, \varphi}$, isto é,

$$
\sup _{\lambda \in K}\left\|\left(\lambda I_{n}-A_{n}\right)^{-1} u_{n}-E_{n}(\lambda I-A)^{-1} u_{0}\right\|_{X_{n}} \stackrel{n \rightarrow \infty}{\rightarrow} 0
$$

Se este não é o caso, existem $\epsilon_{0}>0, X_{n} \ni u_{n} \stackrel{E}{\rightarrow} u_{0} \in X,\left\{\lambda_{k} ; k \in \mathbb{N}\right\} \cup\left\{\lambda_{0}\right\} \subset \Sigma_{\omega, \varphi}$ tais que $\lambda_{k} \rightarrow \lambda_{0} \mathrm{e}$

$$
\left\|\left(\lambda_{k} I_{n_{k}}-A_{n_{k}}\right)^{-1} u_{n_{k}}-E_{n_{k}}\left(\lambda_{k} I-A\right)^{-1} u_{0}\right\|_{X_{n_{k}}} \geq \epsilon_{0}, \forall k \in \mathbb{N} .
$$


Por outro lado,

$$
\begin{aligned}
& \left(\lambda_{k} I_{n_{k}}-A_{n_{k}}\right)^{-1} u_{n_{k}}-E_{n_{k}}\left(\lambda_{k} I-A\right)^{-1} u_{0}= \\
& {\left[\left(\lambda_{k} I_{n_{k}}-A_{n_{k}}\right)^{-1}-\left(\lambda_{0} I_{n_{k}}-A_{n_{k}}\right)^{-1}\right] u_{n_{k}}+\left(\lambda_{0} I_{n_{k}}-A_{n_{k}}\right)^{-1} u_{n_{k}}-E_{n_{k}}\left(\lambda_{0} I-A\right)^{-1} u_{0}} \\
& \quad+E_{n_{k}}\left[\left(\lambda_{0}-A\right)^{-1}-\left(\lambda_{k}-A\right)^{-1}\right] u_{0}= \\
& \begin{array}{r}
{\left[\left(\lambda_{0}-\lambda_{k}\right)\left(\lambda_{k} I_{n_{k}}-A_{n_{k}}\right)^{-1}\left(\lambda_{0} I_{n_{k}}-A_{n_{k}}\right)^{-1}\right]} \\
u_{n_{k}}+\left(\lambda_{0} I_{n_{k}}-A_{n_{k}}\right)^{-1} u_{n_{k}}-E_{n_{k}}\left(\lambda_{0} I-A\right)^{-1} u_{0} \\
\\
\quad+E_{n_{k}}\left(\lambda_{k}-\lambda_{0}\right)\left[\left(\lambda_{0}-A\right)^{-1}\left(\lambda_{k}-A\right)^{-1}\right] u_{0}
\end{array}
\end{aligned}
$$

e assim segue da limitação uniforme dos resolventes, da limitação uniforme da família $\left\{E_{n}\right\}$, e de (1.18) que

$$
\left\|\left(\lambda_{k} I_{n_{k}}-A_{n}\right)^{-1} u_{n_{k}}-E_{n_{k}}(\lambda I-A)^{-1} u_{0}\right\|_{X_{n_{k}}} \stackrel{k \rightarrow \infty}{\rightarrow} 0
$$

que contradiz (1.21). Logo segue a afirmação de EE-convergência uniforme dos resolventes.

Neste caso temos pelo Teorema 0.0.4

$$
e^{A_{n} t}=\frac{1}{2 \pi i} \int_{\Gamma} e^{\lambda t}\left(\lambda I_{n}-A_{n}\right)^{-1} d \lambda
$$

onde $\Gamma$ é a fronteira de $\Sigma_{\omega, \phi}-\{\lambda \in \mathbb{C} ;|\lambda-\omega| \leq r\}$ e $\frac{\pi}{2}<\phi<\varphi$, r pequeno, orientada no setido da parte imaginária crescente e $t \in \mathbb{C} \operatorname{com}|\arg (t)| \leq \epsilon_{1}<\phi-\frac{\pi}{2}$. Logo,

$$
\left\|e^{A_{n} t} u_{n}-E_{n} e^{A t} u_{0}\right\|_{X_{n}} \leq \frac{1}{2 \pi} \int_{\Gamma}\left\|e^{\lambda t}\left(\left(\lambda I_{n}-A_{n}\right)^{-1} u_{n}-E_{n}(\lambda I-A)^{-1} u_{0}\right)\right\| d|\lambda|
$$

Por outro lado, dados $\epsilon, \epsilon_{0}>0$, existe $N>0$ tal que

$$
\frac{1}{2 \pi} \int_{K_{N}}\left\|e^{\lambda t}\left(\left(\lambda I_{n}-A_{n}\right)^{-1} u_{n}-E_{n}(\lambda I-A)^{-1} u_{0}\right)\right\| d|\lambda| \leq \frac{\epsilon}{2},
$$

para todo $t \in \mathbb{C}$ tal que $|\arg (t)| \leq \epsilon_{1}$ e $|t| \geq \epsilon_{0}$. O conjunto $K_{N}$ é definido por

$$
K_{N}=\Gamma \cap\{\lambda \in \mathbb{C} / \operatorname{Re}(\lambda)<-N\}
$$

De fato, para $N$ grande e $\lambda \in K_{N}$ temos

$$
\left\|e^{\lambda t}\left(\lambda I_{n}-A_{n}\right)^{-1}\right\| \leq \frac{M e^{-|\lambda| k_{1} \epsilon_{0}}}{|\lambda-\omega|}, \forall n \in \mathbb{N}
$$

com $k_{1}=\left|\cos \left(\phi-\epsilon_{1}\right)\right|>0$. Assim, concluimos a afirmação acima notando que a família $\left\{E_{n}\right\}$ é uniformemente limitada.

Segue da afirmação anterior que é suficiente mostrarmos que para n suficientemente grante temos

$$
\frac{1}{2 \pi} \int_{J_{N}}\left\|e^{\lambda t}\left(\left(\lambda I_{n}-A_{n}\right)^{-1} u_{n}-E_{n}(\lambda I-A)^{-1} u_{0}\right)\right\| d|\lambda| \leq \frac{\epsilon}{2}
$$


onde $J_{N}=\Gamma-K_{N}$. De fato, como $J_{N}$ é compacto e $J_{N} \subset \Sigma_{\omega, \varphi}$ segue que

$$
\sup _{\lambda \in J_{N}}\left\|\left(\lambda I_{n}-A_{n}\right)^{-1} u_{n}-E_{n}(\lambda I-A)^{-1} u_{0}\right\| \stackrel{n \rightarrow \infty}{\rightarrow} 0
$$

Como $\left|e^{\lambda t}\right|$ é limitada se $\lambda \in J_{N}$ e $t \in \Sigma\left(\theta, \epsilon_{0}, \mu\right)$, segue que existe $n_{0} \in \mathbb{N}$ tal que $n \geq n_{0}$ implica

$$
\sup _{t \in \Sigma\left(\theta, \epsilon_{0}, \mu\right)}\left\|e^{A_{n} t} u_{n}-E_{n} e^{A t} u\right\|_{X_{n}} \leq \epsilon
$$

Logo

$$
\sup _{t \in \Sigma\left(\theta, \epsilon_{0}, \mu\right)}\left\|e^{A_{n} t} u_{n}-E_{n} e^{A t} u\right\|_{X_{n}} \stackrel{n \rightarrow \infty}{\rightarrow} 0
$$

e portanto concluimos que (1.18) implica (1.19).

Reciprocamente, Suponha (1.19). Segue do Teorema 0.0.2 que

$$
\left\|\left(\lambda I_{n}-A_{n}\right)^{-1} u_{n}-E_{n}(\lambda I-A)^{-1} u_{0}\right\|_{X_{n}}=\left\|\int_{0}^{\infty} e^{\lambda t}\left(e^{A_{n} t} u_{n}-E_{n} e^{A t} u_{0}\right) d t\right\|_{X_{n}}
$$

Como $\left\{E_{n}\right\}$ é uniformemente limitada, $\left\{u_{n}\right\}$ é limitada e $\forall t \geq 0, \forall n \in \mathbb{N},\left\|e^{A_{n} t}\right\| \leq M e^{\omega t}$ e $\left\|e^{A t}\right\| \leq M e^{\omega t}$ segue que existe $N>0$ tal que para $\lambda \in \mathbb{C} \operatorname{com} \operatorname{Re}(\lambda)>\omega$,

$$
\left\|\int_{0}^{\frac{1}{N}} e^{\lambda t}\left(e^{A_{n} t} u_{n}-E_{n} e^{A t} u_{0}\right) d t+\int_{N}^{\infty} e^{\lambda t}\left(e^{A_{n} t} u_{n}-E_{n} e^{A t} u_{0}\right) d t\right\|_{X_{n}}<\frac{\epsilon}{2}
$$

Como

$$
\sup _{\frac{1}{N} \leq t \leq N}\left\|e^{A_{n} t} u_{n}-E_{n} e^{A t} u_{0}\right\|_{X_{n}} \stackrel{n \rightarrow \infty}{\rightarrow} 0
$$

e para cada $\lambda,\left|e^{\lambda t}\right|$ é limitada se $t \in[0, N]$, segue (1.18). Note que neste caso obtemos EE-convergência uniforme para $\lambda$ em conjuntos da forma $\{\lambda \in \mathbb{C} / a \leq \operatorname{Re}(\lambda) \leq b\}$ desde que $b \geq a>\omega$. 


\section{Capítulo 2}

\section{Noções de Homogeneização}

Neste capíulo apresentamos as noções básicas de homogeneização de equações diferenciais que faremos uso para se estudar o compotamento das soluções de (8). Apresentamos também os espaços de Sobolev e finalizamos o capítulo com um teorema de convergência baseado no método de Tartar das funções-teste oscilantes. Algumas referências para este assunto são [12], [13], [15], [16].

A principal referência para este capítulo é [13]. Nesta referência encontra-se a prova de alguns resultados listados aqui. Outros cuja prova não se encontra nesta referência são resultados de análise funcional cuja demonstração é simples ou resultados clássicos sobre espaços de Sobolev.

Nesta dissertação, para $\Omega \subset \mathbb{R}^{n}$, os espaços $L^{p}(\Omega)$ são definidos da seguinte maneira:

- Se $1 \leq p<\infty$,

$$
L^{p}(\Omega):=\left\{f: \Omega \rightarrow \mathbb{R} \text { mensuráveis e tais que } \int_{\Omega}|f(x)|^{p} d x<\infty\right\}
$$

onde a integral acima é relativa à medida de Lebesgue. A função $\|\cdot\|_{p}: L^{p}(\Omega) \rightarrow \mathbb{R}^{+}$ definida por

$$
\|u\|_{p}: L^{p}(\Omega)=\left(\int_{\Omega}|u(x)|^{p} d x\right)^{\frac{1}{p}}, \quad u \in L^{p}(\Omega),
$$

é uma norma e o espaço $L^{p}(\Omega)$ com esta norma é um espaço de Banach separável que é também reflexivo se $p>1$.

- Se $p=\infty$, definimos

$L^{\infty}(\Omega):=\{f: \Omega \rightarrow \mathbb{R}$ mensuráveis e tais que existe $C \in \mathbb{R}$ com $|f| \leq C$, q.s. em $\Omega\}$. 
A função $\|\cdot\|_{p}: L^{\infty}(\Omega) \rightarrow \mathbb{R}^{+}$definida por

$$
\|u\|_{\infty}: L^{\infty}(\Omega)=\inf \{c \geq 0:|u(x)| \leq c \text { q.s. em } \Omega\}, \quad u \in L^{\infty}(\Omega)
$$

é uma norma e o espaço $L^{\infty}(\Omega)$ com esta norma é um espaço de Banach que não é separável nem reflexivo.

- Os espaços duais dos espaços $L^{p}(\Omega)$ são caracterizados da seguinte maneira: $\left(L^{p}(\Omega)\right)^{*}=$ $L^{p^{\prime}}(\Omega)$ se $1 \leq p<\infty$ e $p^{\prime}$ definido por $\frac{1}{p}+\frac{1}{p^{\prime}}=1$ se $1<p<\infty$ e $p^{\prime}=\infty$ se $p=1$.

\subsection{Introdução à Homogeneização}

Nesta seção, $\Omega$ denotará um aberto limitado de $\mathbb{R}^{n}$ e $\epsilon>0$ é um parâmetro tomando valores numa sequência que tende a zero.

Seja

$$
A^{\epsilon}(x)=\left(a_{i j}^{\epsilon}(x)\right)_{1 \leq i, j, n} \text { q.s em } \Omega
$$

onde a notação q.s é uma abreviação de quase sempre, isto é, a igualdade é válida exceto num conjunto de medida de Lebesgue nula.

Sejam $\alpha, \beta$ números reais positivos com $\alpha<\beta$. Diremos que

$$
A^{\epsilon} \in M(\alpha, \beta, \Omega)
$$

se $A^{\epsilon}$ satisfaz

$$
\left\{\begin{array}{l}
\left\langle A^{\epsilon} \lambda, \lambda\right\rangle \geq \alpha|\lambda|^{2} \\
\left|A^{\epsilon}(x) \lambda\right| \leq \beta|\lambda|
\end{array}\right.
$$

q.s em $\Omega$ e para todo $\lambda \in \mathbb{R}^{n}$.

Introduzimos o operador

$$
\mathcal{A}_{\epsilon}=-\operatorname{div}\left(A^{\epsilon} \nabla\right)=-\sum_{i, j=1}^{n} \frac{\partial}{\partial x_{i}}\left(a_{i j}^{\epsilon} \frac{\partial}{\partial x_{j}}\right) .
$$

A teoria de homogeneização serve para descrever o comportamento assimtótico quando $\epsilon \rightarrow 0$ de equações diferenciais parciais com operadores diferenciais da forma $\mathcal{A}_{\epsilon}$.

Faremos uma breve discussão sobre o problema de valor de fronteira

$$
\left\{\begin{array}{c}
-\operatorname{div}\left(A^{\epsilon} \nabla u^{\epsilon}\right)=f \text { em } \Omega \\
u^{\epsilon}=0 \text { sobre } \partial \Omega
\end{array}\right.
$$

onde $f \in H^{1}(\Omega)$. 
Segue do Teorema de Lax-Milgram que para cada $\epsilon>0$ existe um único $u^{\epsilon} \in H_{0}^{1}(\Omega)$ tal que

$$
\int_{\Omega} A^{\epsilon} \nabla u^{\epsilon} \nabla v d x=\langle f, v\rangle, \forall v \in H_{0}^{1}(\Omega)
$$

Mais ainda, temos a seguinte estimativa

$$
\left\|u^{\epsilon}\right\|_{H_{0}^{1}(\Omega)} \leq \frac{1}{\alpha}\|f\|_{L^{2}(\Omega)} .
$$

Consequentemente, como $H_{0}^{1}(\Omega)$ é reflexivo, segue que existe uma subsequência $\left\{u^{\epsilon^{\prime}}\right\}$ e um elemento $u^{0} \in H_{0}^{1}(\Omega)$ tal que

$$
u^{\epsilon^{\prime}} \rightarrow u^{0} \operatorname{em~} H_{0}^{1}(\Omega)
$$

Note que, a priori, o limite $u^{0}$ pode depender de da subsequência $u^{\epsilon^{\prime}}$ comada para que (2.8) se verifique.

Neste ponto temos algumas questões naturais:

- $u^{0}$ é unicamente determinada?

- Como determinar $u^{0}$ ?

- $u^{0}$ satisfaz algum problema de valor de fronteira em $\Omega$ ?

No que se segue vamos discutir possíveis respostas para as questões acima.

Nas considerações que faremos a seguir, vamos utilizar a seguinte notação:

$$
\xi^{\epsilon}=\left(\xi_{1}^{\epsilon}, \ldots, \xi_{n}^{\epsilon}\right)=\left(\sum_{j=1}^{n} a_{1 j}^{\epsilon} \frac{\partial u^{\epsilon}}{\partial x_{j}}, \ldots, \sum_{j=1}^{n} a_{n j}^{\epsilon} \frac{\partial u^{\epsilon}}{\partial x_{j}}\right)=A^{\epsilon} \nabla u^{\epsilon} .
$$

Note que

$$
\int_{\Omega} \xi^{\epsilon} \nabla v d x=\langle f, v\rangle, \forall v \in H_{0}^{1}(\Omega)
$$

Segue de (2.2) e (2.7) que

$$
\left\|\xi^{\epsilon}\right\|_{\left(L^{2}(\Omega)\right)^{n}} \leq \frac{\beta}{\alpha}\|f\|_{L^{2}(\Omega)}
$$

Como $\left(L^{2}(\Omega)\right)^{n}$ é reflexivo, segue que existe uma subsequência $\left\{\xi^{\epsilon^{\prime}}\right\}$, e um elemento $\xi^{0} \in\left(L^{2}(\Omega)\right)^{n}$ tal que

$$
\xi^{\epsilon^{\prime}} \rightarrow \xi^{0} \mathrm{em}\left(L^{2}(\Omega)\right)^{n}
$$

Assim, por passagem ao limite em (2.10) pela subsequência $\epsilon^{\prime}$ obtemos

$$
\int_{\Omega} \xi^{0} \nabla v d x=\langle f, v\rangle, \forall v \in H_{0}^{1}(\Omega) .
$$


Consequentemente a primeira questão tem uma resposta positiva, se pudermos descrever $\xi_{0}$ em termos de $u^{0}$.

Observamos que se $A^{\epsilon}$ é tal que

$$
A^{\epsilon} \rightarrow \bar{A} \mathrm{em}\left(L^{\infty}(\Omega)\right)^{n^{2}}
$$

então $\xi_{0}=\bar{A} \nabla u^{0}$ e portanto segue que $u^{0}$ é a única solução (do Teorema de Lax-Milgram) da formulação variacional do problema

$$
\left\{\begin{array}{c}
-\operatorname{div}\left(\bar{A} \nabla u^{0}\right)=f \text { em } \Omega \\
u^{0}=0 \text { sobre } \partial \Omega
\end{array}\right.
$$

isto é,

$$
\int_{\Omega} \bar{A} \nabla u^{0} \nabla v d x=\langle f, v\rangle, \forall v \in H_{0}^{1}(\Omega)
$$

Quando a convergência de $A^{\epsilon}$ para $\bar{A}$ ocorre em topologias mais fracas as considerações acima não se aplicam e o estudo se torna bastante mais elaborado. Vamos agora considerar uma situação específica na qual a convergência de $A^{\epsilon}$ para $\bar{A}$ ocorre apenas na topologia fraca* de $\left(L^{\infty}(\Omega)\right)^{n^{2}}$ para mostrar as dificuldades e técnicas envolvidas. O caso que consideraremos é o caso de funções periódicas com freqüência tendendo a infinito.

Seja $Y$ um intervalo de $\mathbb{R}^{n}$ definido por

$$
\left(0, l_{1}\right) \times \cdots \times\left(0, l_{n}\right)
$$

onde $l_{1}, \ldots, l_{n}$ são números positivos. Iremos nos referir a $Y$ como uma célula de referência. Definição 2.1.1. Seja $Y$ dado pela equação (2.14) e seja $f$ uma função definida q. s. em $\mathbb{R}^{n}$. A função f é chamada de Y-periódica se

$$
f\left(x+k l_{i} e_{i}\right)=f(x) \text { q. s. em } \mathbb{R}^{n}, \forall k \in \mathbb{Z}, i \in\{1, \ldots, n\},
$$

onde $e_{1}, \ldots, e_{n}$ é a base canônica de $\mathbb{R}^{n}$. No caso $n=1$, simplesmente dizemos que $f$ é $l_{1}$-periódica.

Definição 2.1.2. Seja $\Omega$ um aberto limitado de $\mathbb{R}^{n}$ e $f$ uma função em $L^{1}(\Omega)$. O valor médio de $f$ em $\Omega$ é o número real dado por

$$
M_{\Omega}(f)=\frac{1}{|\Omega|} \int_{\Omega} f(y) d y .
$$


Sejam $\alpha, \beta \in \mathbb{R}$, tais que $\alpha<\beta$ e $A=\left(a_{i j}\right)_{1 \leq i, j \leq n}$ uma matriz $n \times n$ tal que

$$
\left\{\begin{array}{l}
a_{i j} \text { é Y-periódica, } i, j=1, \ldots, n \\
A \in M(\alpha, \beta, Y)
\end{array}\right.
$$

Seja

$$
a_{i j}^{\epsilon}(x)=a_{i j}\left(\frac{x}{\epsilon}\right) \text { q. s. em } \mathbb{R}^{n}, \forall i, j=1, \ldots, n
$$

e

$$
A^{\epsilon}(x)=A\left(\frac{x}{\epsilon}\right)=\left(a_{i j}^{\epsilon}(x)\right)_{1 \leq i, j \leq n} \text { q. s. em } \mathbb{R}^{n} .
$$

É simples verificar que $A^{\epsilon}$ satisfaz (2.2) para todo $\epsilon$. Então todas as considerações acima valem para o problema (2.5) escrito com $A^{\epsilon}$ dado por (2.17).

Segue do Teorema 2.2.1 (da próxima seção) que se $\epsilon \rightarrow 0$, então

$$
A^{\epsilon} \stackrel{*}{\rightarrow} M_{Y}(A) \operatorname{em}\left(L^{\infty}(\Omega)\right)^{n^{2}}
$$

onde a matriz $\left(M_{Y}(A)\right)_{i j}$ é definida por

$$
\left(M_{Y}(A)\right)_{i j}=\frac{1}{|Y|} \int_{Y} a_{i j}(y) d y
$$

Neste caso, é natural perguntar se $\xi^{0}=M_{Y}(A) \nabla u^{0}$. Esta igualdade é falsa mesmo em dimensão 1. Em dimensão 1 temos $u^{0} \in H_{0}^{1}(b, c)$ única solução da formulação variacional do seguinte problema

$$
\left\{\begin{array}{l}
-\frac{d}{d x}\left(\frac{1}{M_{\left(0, l_{1}\right)}\left(\frac{1}{a}\right)} \frac{d u^{0}}{d x}\right)=f \text { em }(b, c) \\
u^{0}(b)=u^{0}(c)=0
\end{array}\right.
$$

Como em geral temos

$$
\frac{1}{M_{\left(0, l_{1}\right)}\left(\frac{1}{a}\right)} \frac{d u^{0}}{d x} \neq M_{\left(0, l_{1}\right)}(a) \frac{d u^{0}}{d x}
$$

segue que

$$
\lim _{\epsilon \rightarrow 0}\left(a^{\epsilon} \frac{d u^{\epsilon}}{d x}\right) \neq\left(\lim _{\epsilon \rightarrow 0} a^{\epsilon}\right)\left(\lim _{\epsilon \rightarrow 0} \frac{d u^{\epsilon}}{d x}\right)
$$

no sentido de convergência fraca em $L^{2}(b, c)$.

Nas próximas seções, apresentaremos condições sufucientes para obtermos o problema limite e a convergência das soluções. 


\subsection{Funções periódicas altamente oscilantes}

O objetivo desta seção é demonstrar o Teorema 2.2.1 que caracteriza a convergência fraca e fraca* de funções periódicas. Para isto precisaremos dos seguintes resultados auxiliares.

Lema 2.2.1. Seja $f$ uma função Y-periódica em $L^{1}(Y)$. Seja y ym ponto fixado em $\mathbb{R}^{n} e$ denote por $Y_{0}$ a translação de $Y$ definida por

$$
Y_{0}=y_{0}+Y
$$

Seja

$$
f_{\epsilon}(x)=f\left(\frac{x}{\epsilon}\right) \text { q. s. em } \mathbb{R}^{n} \text {. }
$$

Então

$$
\left\{\begin{array}{l}
\text { i) } \int_{Y_{0}} f(y) d y=\int_{Y} f(y) d y \\
\text { ii) } \int_{\epsilon Y_{0}} f_{\epsilon}(y) d y=\int_{\epsilon Y} f_{\epsilon}(y) d y=\epsilon^{n} \int_{Y} f(y) d y .
\end{array}\right.
$$

Lema 2.2.2. Seja E um espaço de Banach reflexivo e $\left\{x_{n}\right\}$ uma sequência limitada em E. Então

i) Existe uma subsequência $\left\{x_{n_{k}}\right\}$ de $\left\{x_{n}\right\}$ e $x \in E$ tal que $\left\{x_{n_{k}}\right\}$ converge fracamente para $x$. ii) Se toda subsequência fracamente convergente de $\left\{x_{n}\right\}$ tem o mesmo limite $x$, então a sequência $\left\{x_{n}\right\}$ converge fracamente para $x$.

Prova: A prova de i) pode ser encontrada em [5]

Apresentamos aqui a prova de ii). De fato, suponha que toda subsequência de $\left\{x_{n}\right\}$ fracamente convergente converge para $x \in E$. Se $\left\{x_{n}\right\}$ não converge fracamente para $x$, então existem $\epsilon_{0}>0, x^{*} \in E^{*}$ e uma subsequência $\left\{x_{n_{k}}\right\}$ tal que $\left|x^{*}\left(x_{n_{k}}-x\right)\right| \geq \epsilon_{0}$ e isto contradiz o item i) pois para $\left\{x_{n_{k}}\right\}$ deve ter uma subsequência fracamente convergênte para $x$.

Lema 2.2.3. Seja $1<p<\infty$ e $\left\{u_{n}\right\}$ uma sequência em $L^{p}(\Omega)$. Então as seguintes condições são equivalentes:

1. $u_{n} \rightarrow u$ em $L^{p}(\Omega)$.

2. Existe $C \geq 0$ tal que $\left\|u_{n}\right\|_{L^{p}(\Omega)} \leq C, \forall n \in \mathbb{N} e$

$$
\int_{I} u_{n} d x \rightarrow \int_{I} u d x, \text { para todo intervalo } I \subset \Omega \text {. }
$$


Prova: 1) $\Rightarrow$ 2) trivial.

2 ) $\Rightarrow$ 1). Segue de [7] (Teorema 2.40 (c), seção 2.6 combinado com a Proposição 6.7, seção 6.1) que o conjunto das funções simples $f=\sum_{j=1}^{n} a_{j} \chi_{I_{j}}$ com $I_{j} \in \Omega, I_{j}$ intervalo e $a_{j} \in \mathbb{R}$, é denso em $L^{q}(\Omega)$ para todo $1 \leq q<\infty$. Seja $f=\lim f_{k}\left(\operatorname{em~} L^{q}(\Omega) \operatorname{com} \frac{1}{q}+\frac{1}{p}=1\right)$ com cada $f_{k}=\sum_{j=1}^{j_{k}} a_{j, k} \chi_{I_{j, k}}, I_{j, k}$ intervalo contido em $\Omega$. Como

$$
\int_{\Omega} f\left(u_{n}-u\right) d x=\int_{\Omega}\left(f-f_{k}\right)\left(u_{n}-u\right) d x+\int_{\Omega} f_{k}\left(u_{n}-u\right) d x
$$

podemos, para cada $\epsilon>0$, escolher $k_{0} \in \mathbb{N}$ tal que

$$
\left|\int_{\Omega}\left(f-f_{k_{0}}\right)\left(u_{n}-u\right) d x\right| \leq\left\|f-f_{k_{0}}\right\|_{L^{q}(\Omega)}\left\|u_{n}-u\right\|_{L^{p}(\Omega)} \leq \frac{\epsilon}{2} \forall n \in \mathbb{N} .
$$

Fixado $k_{0}$ como acima, existe $n_{0} \in \mathbb{N}$ tal que

$$
\left|\int_{\Omega} f_{k_{0}}\left(u_{n}-u\right) d x\right|<\frac{\epsilon}{2} \forall n \geq n_{0}
$$

e portanto

$$
\left|\int_{\Omega} f\left(u_{n}-u\right) d x\right|<\epsilon \forall n \geq n_{0}
$$

e isto completa a prova.

Com os lemas anteriores, podemos estabelecer o seguinte teorema.

Teorema 2.2.1. Seja $1 \leq p \leq \infty$ e f uma função $Y$-periódica em $L^{p}(Y)$. Seja

$$
f_{\epsilon}(x)=f\left(\frac{x}{\epsilon}\right) q . s . e m \mathbb{R}^{n} .
$$

Nestas condições, se $1 \leq p<\infty$ quando $\epsilon \rightarrow 0$, então

$$
f_{\epsilon} \rightarrow M_{Y}(f)=\frac{1}{|Y|} \int_{Y} f(y) d y \operatorname{em~} L^{p}(\omega)
$$

para qualquer aberto limitado $\omega$ em $\mathbb{R}^{n}$.

Se $p=\infty$, então

$$
f_{\epsilon} \stackrel{*}{\rightarrow} M_{Y}(f)=\frac{1}{|Y|} \int_{Y} f(y) d y \text { em } L^{\infty}\left(\mathbb{R}^{n}\right)
$$


Prova: A prova será feita em duas etapas.

Estimativa a priori: Nesta etapa buscaremos obter uma estimativa apriori para a família $\left\{f_{\epsilon}\right\}$ que nos permita extrair uma subseqüência convergente da mesma. Vamos considerar os casos $p=\infty$ e $1 \leq p<\infty$ separadamente.

Se $p=\infty$, levando em conta (2.22), temos

$$
\left\|f_{\epsilon}\right\|_{L^{\infty}\left(\mathbb{R}^{n}\right)}=\|f\|_{L^{\infty}(Y)} .
$$

Como conseqúência desta estimativa apriori e do fato que a a bola fechada de raio $\|f\|_{L^{\infty}(Y)}$ em $L^{\infty}\left(\mathbb{R}^{n}\right)$ é pré-compacta na topologia fraca* de $L^{\infty}\left(\mathbb{R}^{n}\right)$, podemos extrair uma subsequência $\left\{f_{\epsilon^{\prime}}\right\}$ tal que

$$
f_{\epsilon^{\prime}} \stackrel{*}{\rightarrow} F \text { em } L^{\infty}\left(\mathbb{R}^{n}\right)
$$

Considere agora o caso $p<\infty$ e $\omega$ um subconjunto limitado de $\mathbb{R}^{n}$. Para obtermos uma estimativa a priori em $L^{p}(\omega)$, é suficiente mostrarmos que para qualquer retângulo $I$ de $\mathbb{R}^{n}$, existe uma constante $C$ independente de $\epsilon$ tal que

$$
\left\|f_{\epsilon}\right\|_{L^{p}(I)} \leq C
$$

Sem perda de generalidade podemos supor que $I$ contém ao menos um conjunto transladado de $Y$.

Note que podemos encontrar $N(\epsilon)$ conjuntos transladados de $Y$ disjuntos denotados por $Y_{k}, k=1, \ldots, N(\epsilon)$ com $\epsilon Y_{k} \subset I$, e $N^{\prime}(\epsilon)$ conjuntos transladados de $Y$ disjuntos denotados por $Y_{j}^{\prime}, j=1, \ldots, N^{\prime}(\epsilon) \operatorname{com} \epsilon Y_{j}^{\prime} \cap \partial I \neq \emptyset$, tal que

$$
I \subset\left(\bigcup_{k=1}^{N(\epsilon)} \epsilon \overline{Y_{k}}\right) \cup\left(\bigcup_{j=1}^{N^{\prime}(\epsilon)} \epsilon \overline{Y_{j}^{\prime}}\right)
$$

Sejam $L_{1}, \ldots, L_{n}$ o comprimento dos lados de $I$. Provemos primeiramente que

$$
\left\{\begin{array}{l}
\text { i) } \lim _{\epsilon \rightarrow 0} \epsilon^{n} N(\epsilon)=\frac{|I|}{|Y|} \\
\text { ii) } \lim _{\epsilon \rightarrow 0} \epsilon^{n-1} N^{\prime}(\epsilon) \leq \sum_{i=1}^{n}\left(\prod_{j \neq i} \frac{L_{j}}{l_{j}}\right) \leq n \frac{|I|}{|Y|} .
\end{array}\right.
$$

Note que para cada $\epsilon>0$ existem $k_{1}^{\epsilon}, \ldots, k_{n}^{\epsilon} \in \mathbb{N}$ tais que, para todo $i=1, \ldots, n$,

$$
L_{i}=\epsilon k_{i}^{\epsilon} l_{i}+\gamma_{i}^{\epsilon} \operatorname{com} 0 \leq \gamma_{i}^{\epsilon}<\epsilon l_{i}
$$

e consequentemente,

$$
\epsilon k_{i}^{\epsilon} \rightarrow \frac{L_{i}}{l_{i}} \text { quando } \epsilon \rightarrow 0
$$


Por outro lado, note que o número de conjuntos transladados de $\epsilon Y$ contidos no interior de $I$ é

$$
N(\epsilon)=k_{1}^{\epsilon} \times \cdots \times k_{n}^{\epsilon}
$$

que junto com (2.26) implica que

$$
\epsilon^{n} N(\epsilon) \rightarrow \frac{L_{1} \times \cdots \times L_{n}}{l_{1} \times \cdots \times l_{n}}=\frac{|I|}{|L|}
$$

que é a afirmação $i$ ). Para avaliar $N^{\prime}(\epsilon)$ note que os conjuntos $Y_{k}^{\prime}$ e $Y_{j}$ podem ser escolhidos tais que o retângulo $I$ é coberto pela união de $K(\epsilon)$ conjuntos transladados de $\epsilon \bar{Y}$ disjuntos $\operatorname{com} K(\epsilon)=\left(k_{1}^{\epsilon}+1\right) \times \cdots \times\left(k_{n}^{\epsilon}+1\right)$. Então

$$
N^{\prime}(\epsilon) \leq K(\epsilon)-N(\epsilon)=A_{\epsilon}+B_{\epsilon}
$$

onde

$$
A_{\epsilon}=\sum_{i=1}^{n}\left(\prod_{i \neq j} k_{j}^{\epsilon}\right)
$$

De (2.26) obtemos

$$
\epsilon^{n-1} A_{\epsilon} \rightarrow \sum_{i=1}^{n}\left(\prod_{j \neq i} \frac{L_{j}}{l_{j}}\right) \leq n \prod_{j=1}^{n} \frac{L_{j}}{l_{j}}=n \frac{|I|}{|Y|}
$$

pois para todo $j=1, \ldots, n$, temos $l_{j}<L_{j}$. Isto implica o item $i i$ ) pois por construção temos

$$
\epsilon^{n-1} B_{\epsilon} \rightarrow 0
$$

Agora, da periodicidade de $f$, do Lema 2.2.1, e das estimativas (2.25) temos

$$
\left\{\begin{aligned}
\left\|f_{\epsilon}\right\|_{L^{p}(I)}^{p} & \leq \sum_{k=1}^{N(\epsilon)} \int_{\epsilon Y_{k}}\left|f_{\epsilon}\right|^{p} d x+\sum_{j=1}^{N^{\prime}(\epsilon)} \int_{\epsilon Y_{j}^{\prime}}\left|f_{\epsilon}\right|^{p} d x \\
& =\left[N(\epsilon)+N^{\prime}(\epsilon)\right] \int_{\epsilon Y}\left|f_{\epsilon}\right|^{p} d x \\
& =\left[N(\epsilon)+N^{\prime}(\epsilon)\right] \epsilon^{n} \int_{Y}|f(y)|^{p} d y \leq C\|f\|_{L^{p}(Y)}^{p},
\end{aligned}\right.
$$

onde $C$ é uma constante independente de $\epsilon$.

Isto mostra que a sequência $\left\{f_{\epsilon}\right\}$ é limitada em $L^{p}(\omega)$, para qualquer subconjunto limitado $\omega$ de $\mathbb{R}^{n}$ e para qualquer $1 \leq p<\infty$.

No caso particular, $1<p<\infty, L^{p}(\omega)$ é reflexivo e portanto existe uma subsequência $\left\{f_{\epsilon^{\prime}}\right\}$ tal que

$$
f_{\epsilon^{\prime}} \rightarrow G \text { em } L^{p}(\omega) .
$$


Identificação do limite: Nesta etapa mostraremos que o limite encontrado em (2.23) e em (2.28) independe da subseqüência tomada para concluir que o limite existe. Dividiremos a prova nos casos $1<p<\infty, p=\infty$ e $p=1$.

(a) Caso $1<p<\infty$.

Seja $\omega$ um subconjunto aberto limitado de $\mathbb{R}^{n}$. Do passo anterior e do Lema 2.2.3, para identificar o limite em (2.28), é suficiente mostrarmos que

$$
\int_{I} f_{\epsilon}(x) d x \rightarrow \int_{I} M_{Y}(f) d x=|I| M_{Y}(f),
$$

para qualquer retângulo $I \subset \omega$. Usando (2.24) obtemos

$$
\int_{I} f_{\epsilon}(x) d x=N(\epsilon) \epsilon^{n} \int_{Y} f(y) d y+\sum_{i=1}^{N^{\prime}(\epsilon)} \int_{\epsilon Y_{j}^{\prime} \cap I} f_{\epsilon}(x) d x .
$$

De (2.25) segue que

$$
N(\epsilon) \epsilon^{n} \int_{Y} f(y) d y \rightarrow \frac{|I|}{|Y|} \int_{Y} f(y) d y=|Y| M_{Y}(f)
$$

e também

$$
\left|\sum_{j=1}^{N^{\prime}(\epsilon)} \int_{\epsilon Y_{j}^{\prime}} f_{\epsilon}(x) d x\right| \leq N^{\prime}(\epsilon)\left|\int_{\epsilon Y} f_{\epsilon}(y) d y\right|=\epsilon^{n} N^{\prime}(\epsilon)\left|\int_{Y} f(y) d y\right| \rightarrow 0 .
$$

Consequentemente, em (2.28) obtemos $G=M_{Y}(f)$. Mais ainda, (ii) do Lema 2.2.2 implica que a sequência inteira $\left\{f_{\epsilon}\right\}$ converge para $M_{Y}(f)$ fracamente em $L^{p}(\omega)$.

(b) Caso $p=\infty$.

Seja $\omega$ um subconjunto aberto limitado de $\mathbb{R}^{n}$ e $\chi_{\omega}$ a sua função característica. Nestas condições, se $\varphi \in L^{2}(\omega)$, então $\varphi \chi_{\omega} \in L^{1}\left(\mathbb{R}^{n}\right)$. Então de (2.23) obtemos

$$
\int_{\omega} f_{\epsilon^{\prime}} \varphi d x \rightarrow \int_{\omega} F \varphi d x
$$

Assim,

$$
f_{\epsilon^{\prime}} \rightarrow F \text { em } L^{2}(\omega)
$$

Do passo (a) e da unicidade do limite obtemos

$$
F=M_{Y}(f), q . s \text { em } \omega
$$

e portanto q.s em $\mathbb{R}^{n}$ pois $\omega$ é arbitrário. Com isto obtemos que qualquer subsequência $\left\{\epsilon^{\prime}\right\}$ tem subsequência $\left\{\epsilon^{\prime \prime}\right\}$ tal que $f_{\epsilon^{\prime \prime}}{ }^{*} M_{Y}(f)$ e portanto, analogamente ao Lema 2.2 .2 (ii) obtemos que a sequência toda $\left\{f_{\epsilon}\right\}$ converge para $M_{Y}(f)$ na topologia fraca* de $L^{\infty}\left(\mathbb{R}^{n}\right)$. 
(c) caso $p=1$.

Como nem todo subconjunto limitado de $L^{1}$ é fracamente compacto (toda sequência dele tem subsequência fracamente convergente) para provar o resultado neste caso iremos aplicar um argumento de densidade. Como para todo $1 \leq p<\infty, L^{p}(\Omega)$ é denso em $L^{1}(\Omega)$, segue que para cada $\eta>0$ existe $g \in L^{2}(Y)$ tal que

$$
\|f-g\|_{L^{1}(Y)} \leq \eta
$$

Vamos estender $g$ periodicamente em $\mathbb{R}^{n}$ fazendo

$$
g\left(x+k l_{i} e_{i}\right)=g(x) \text { em } Y, \forall k \in \mathbb{Z}, \forall i \in\{i, \ldots, n\},
$$

onde $\left\{e_{1}, \ldots, e_{n}\right\}$ é a base canônica de $\mathbb{R}^{n}$. Definimos a função $g_{\epsilon}$ por

$$
g_{\epsilon}(x)=g\left(\frac{x}{\epsilon}\right) \text { em } \mathbb{R}^{n}
$$

Seja $\omega$ um aberto limitado de $\mathbb{R}^{n}$. Então para todo $\varphi \in L^{\infty}(\omega)$, temos

$$
\begin{aligned}
\int_{\omega}\left(f_{\epsilon}-M_{Y}(f)\right) \varphi d x & =\int_{\omega}\left(f_{\epsilon}-g_{\epsilon}\right) \varphi d x+\int_{\omega}\left(g_{\epsilon}-M_{Y}(g)\right) \varphi d x \\
& +\int_{\omega}\left(M_{Y}(f)-M_{Y}(g)\right) \varphi d x .
\end{aligned}
$$

Se $I$ é um retângulo de $\mathbb{R}^{n}$ tal que $\omega \subset I$, segue de (2.27) e (2.29) que existe uma constante $C_{1}$, independente de $\epsilon$ e $\eta$ tal que

$$
\left|\int_{\omega}\left(f_{\epsilon}-g_{\epsilon}\right) \varphi d x\right| \leq\|\varphi\|_{L^{\infty}(\omega)}\left\|f_{\epsilon}-g_{\epsilon}\right\|_{L^{1}(I)} \leq C_{1} \eta .
$$

Segue imediatamente de (2.29) que

$$
\left|\int_{\omega}\left(M_{Y}(f)-M_{Y}(g)\right) \varphi d x\right| \leq C_{2} \eta
$$

onde $C_{2}$ é uma constante independente de $\epsilon$ e $\eta$. Finalmente, segue do passo (a) quando $\epsilon \rightarrow 0$

$$
\int_{\omega}\left(g_{\epsilon}-M_{Y}(g)\right) \varphi d x \rightarrow 0 .
$$

Consequentemente, segue de (2.30) que

$$
\int_{\omega}\left(f_{\epsilon}-M_{Y}(f)\right) \varphi d x \rightarrow 0
$$

pois $\eta$ é arbitrário. Isto termina a prova do Teorema 2.2.1 


\subsection{Espaços de Sobolev}

Nesta seção enunciamos as noções e resultados básicos relativos aos espaços $L^{p}(\Omega)$ e $W^{m, p}(\Omega)$, com $1 \leq p \leq \infty$ e $m \in \mathbb{N}$ e $\Omega \subset \mathbb{R}^{N}$ é um domínio aberto limitado.

Definição 2.3.1. Seja $f \in L^{p}(\Omega)$. Diremos que $f$ tem derivada fraca com relação a $x_{i}$ em $L^{p}(\Omega)$ se existe $g \in L^{p}(\Omega)$ tal que

$$
\int_{\Omega} f \frac{\partial \phi}{\partial x_{i}} d x=-\int_{\Omega} g \phi d x, \forall \phi \in C_{0}^{1}(\Omega)
$$

É fácil provar que a derivada fraca é única e denotaremos a derivada fraca de $f$ com relação a $x_{i}$ por $\frac{\partial f}{\partial x_{i}}$.

Definição 2.3.2. Seja $1 \leq p \leq \infty$. O espaço de Sobolev $W^{1, p}(\Omega)$ é definido por

$$
W^{1, p}(\Omega)=\left\{u \in L^{p}(\Omega), \frac{\partial u}{\partial x_{i}} \in L^{p}(\Omega), i=1, \ldots, n\right\} .
$$

Para $p=2$, denotamos $W^{2, p}(\Omega)=H^{1}(\Omega)$.

Proposição 2.3.1. 1. O espaço $W^{1, p}(\Omega)$ é um espaço de Banach com a norma

$$
\|u\|_{W^{1, p}(\Omega)}=\|u\|_{L^{p}(\Omega)}+\sum_{i=1}^{n}\left\|\frac{\partial u}{\partial x_{i}}\right\|_{L^{p}(\Omega)} .
$$

Para $1 \leq p<\infty$, esta norma é equivalente a seguinte norma

$$
\|u\|_{W^{1, p}(\Omega)}=\left(\|u\|_{L^{p}(\Omega)}^{p}+\|\nabla u\|_{L^{p}(\Omega)}^{p}\right)^{\frac{1}{p}}
$$

onde

$$
\nabla u:=\left(\frac{\partial u}{\partial x_{1}}, \ldots, \frac{\partial u}{\partial x_{n}}\right)
$$

2. O espaço $W^{1, p}(\Omega)$ é separável para $1 \leq p<\infty$ e reflexivo para $1<p<\infty$.

3. O espaço $H^{1}(\Omega)$ é um espaço de Hilbert para o produto escalar

$$
(u, v)_{H^{1}(\Omega)}=(u, v)_{L^{2}(\Omega)}+\sum_{i=1}^{n}\left(\frac{\partial u}{\partial x_{i}}, \frac{\partial u}{\partial x_{i}}\right)_{L^{2}(\Omega)}, \forall u, v \in H^{1}(\Omega),
$$

onde

$$
(u, v)_{L^{2}(\Omega)}:=\int_{\Omega} u v d x
$$


Definição 2.3.3. A fronteira $\partial \Omega$ é Lipschitz contínua se existem constantes $c_{1}, c_{2}>0 e$ um número finito $M$ de sistemas de coordenadas locais $\left(\widetilde{x}^{m}, x_{n}^{m}\right)$ e aplicações locais $\varphi_{m}, m=$ $1, \ldots, M$ definidas sobre o conjunto

$$
R_{m}=\left\{\widetilde{x}^{m} \in \mathbb{R}^{n-1} ; \widetilde{x}^{m}=\left(\widetilde{x}_{1}^{m}, \ldots, \widetilde{x}_{n-1}^{m}\right),\left|\widetilde{x}_{i}^{m}\right| \leq c_{1}, \forall i=1, \ldots, n-1\right\},
$$

que são Lipschitz contínuas em seus domínios de definição, tais que:

$$
\partial \Omega=\cup_{m=1}^{M} \Gamma_{m}
$$

onde, para cada $m=1, \ldots, M$,

$$
\Gamma_{m}=\left\{\left(\widetilde{x}^{m}, x_{n}^{m}\right) ; x_{n}^{m}=\varphi_{m}\left(\widetilde{x}^{m}\right), \widetilde{x}^{m} \in R_{m}\right\}
$$

e

$$
\begin{gathered}
\Omega_{m}\left\{\left(\widetilde{x}^{m}, x_{n}^{m}\right) ; \varphi_{m}\left(\widetilde{x}^{m}\right)<x_{n}^{m}<\varphi_{m}\left(\widetilde{x}^{m}\right)+c_{2}, \widetilde{x}^{m} \in R_{m}\right\} \subset \Omega, \\
\mathcal{C}_{m}\left\{\left(\widetilde{x}^{m}, x_{n}^{m}\right) ; \varphi_{m}\left(\widetilde{x}^{m}\right)-c_{2}<x_{n}^{m}<\varphi_{m}\left(\widetilde{x}^{m}\right), \widetilde{x}^{m} \in R_{m}\right\} \subset \mathbb{R}^{n}-\bar{\Omega} .
\end{gathered}
$$

Teorema 2.3.1 (Imersões de Sobolev). Suponha que $\partial \Omega$ é Lipschitz contínua. Então

1. Se $1 \leq p<n, W^{1, p}(\Omega) \subset L^{q}(\Omega)$ com

- inclusão compacta para $q \in\left[1, p^{*}\right)$ onde $\frac{1}{p^{*}}=\frac{1}{p}-\frac{1}{n}$,

- inclusão contínua se $q=p^{*}$,

2. Se $p=n, W^{1, n}(\Omega) \subset L^{q}(\Omega)$ com inclusão compacta se $q \in[1, \infty)$,

3. Se $p>n, W^{1, p}(\Omega) \subset C^{0}(\bar{\Omega})$ com inclusão compacta.

Teorema 2.3.2. Se $\Omega$ é um aberto limitado de $\mathbb{R}^{n}$ com $\partial \Omega$ Lipschitz contínua, então existe uma única transformação linear contínua

$$
\gamma: H^{1}(\Omega) \mapsto L^{2}(\partial \Omega)
$$

tal que para toda $u \in H^{1}(\Omega) \cap C^{0}(\bar{\Omega})$ temos $\gamma(u)=\left.u\right|_{\partial \Omega}$. A função $\gamma(u)$ é chamada o traço de u sobre $\partial \Omega$.

Teorema 2.3.3 (Fórmula de Green). Suponha que $\partial \Omega$ é Lipschitz contínua. Sejam u,v $\in$ $H^{1}(\Omega)$. Então

$$
\int_{\Omega} u \frac{\partial v}{\partial x_{i}} d x=-\int_{\Omega} v \frac{\partial u}{\partial x_{i}} d x+\int_{\partial \Omega} \gamma(u) \gamma(v) N_{i} d s
$$

para $1 \leq i \leq n$ onde $N=\left(N_{1}, \ldots, N_{n}\right)$ denota o vetor normal unitário exterior a $\Omega$. 
Proposição 2.3.2 (Desigualdade de Poincaré-Wirtinger). Seja $\Omega$ um subconjunto conexo limitado de $\mathbb{R}^{n}$. Então existe uma constante $C(\Omega)$ que depende somente do diametro de $\Omega$ tal que

$$
\left\|u-M_{\Omega}(u)\right\|_{L^{2}(\Omega)} \leq C(\Omega)\|\nabla u\|_{\left(L^{2}(\Omega)\right)^{n}}
$$

onde $M_{\Omega}(u)$ é é o valor médio de u em $\Omega$ (veja Definição 2.1.2).

\subsection{Método de Tartar e o Teorema de Convergência}

Nesta seção apresentaremos as funções-teste oscilantes de Tartar e um esboço da demonstração de um teorema de convergência que responde algumas questões, postuladas no início do capítulo, sobre o problema limite quando $\epsilon \rightarrow 0$.

Consideremos novamente o problema

$$
\left\{\begin{array}{c}
-\operatorname{div}\left(A^{\epsilon} \nabla u^{\epsilon}\right)=f \text { em } \Omega \\
u^{\epsilon}=0 \text { sobre } \partial \Omega
\end{array}\right.
$$

com

$$
\begin{aligned}
a_{i j}^{\epsilon}(x) & =a_{i j}\left(\frac{x}{\epsilon}\right) \text { q. s. em } \mathbb{R}^{n}, \forall i, j=1, \ldots, n \\
A^{\epsilon}(x) & =A\left(\frac{x}{\epsilon}\right)=\left(a_{i j}^{\epsilon}(x)\right)_{1 \leq i, j \leq n} \text { q. s. em } \mathbb{R}^{n} .
\end{aligned}
$$

onde

$$
\left\{\begin{array}{l}
a_{i j} \text { é Y-periódica, } i, j=1, \ldots, n \\
A \in M(\alpha, \beta, Y),
\end{array}\right.
$$

com números reais positivos $\alpha$ e $\beta$ tais que $\alpha<\beta$. Aqui, $Y$ é definido da seguinte forma

$$
Y=\left(0, l_{1}\right) \times \cdots \times\left(0, l_{n}\right),
$$

onde $l_{1}, \ldots, l_{n}>0$.

Definição 2.4.1. Seja $C_{\text {per }}^{\infty}(Y)$ o subconjunto de $C^{\infty}\left(\mathbb{R}^{n}\right)$ das funções Y-periódicas. Denotamos por $H_{p e r}^{1}(Y)$ o fecho de $C_{p e r}^{\infty}(Y)$ na norma de $H^{1}(Y)$. Definimos o espaço $W_{p e r}(Y) d a$ seguinte forma

$$
W_{\text {per }}(Y)=\left\{u \in H_{\text {per }}^{1}(Y) ; M_{Y}(u)=0\right\}
$$

Considere para cada $\lambda \in \mathbb{R}^{n}$ a solução do seguinte problema

$$
\left\{\begin{array}{c}
-\operatorname{div}\left(A(y) \nabla \widehat{\chi}_{\lambda}\right)=-\operatorname{div}(A(y) \lambda) \text { em } Y \\
\widehat{\chi}_{\lambda} \in W_{p e r}(Y)
\end{array}\right.
$$


com a seguinte formulação

$$
\left\{\begin{array}{l}
\text { Encontrar } \widehat{\chi}_{\lambda} \in W_{\text {per }}(Y) \text { tal que } \\
a_{Y}\left(\widehat{\chi}_{\lambda}, v\right)=\int_{Y} A \lambda \nabla v d y, \forall v \in W_{p e r}(Y) .
\end{array}\right.
$$

onde

$$
a_{Y}(u, v)=\int_{Y} A \nabla u \nabla v d y, \forall u, v \in W_{p e r}(Y)
$$

Segue do Teorema de Lax-Milgram que o problema (2.36) tem uma única solução.

Considere a extensão de $\widehat{\chi}_{\lambda}$ periodicamente para todo $\mathbb{R}^{n}$ e denotemos também por $\widehat{\chi}_{\lambda}$. Segue do Teorema 4.28 de [13] que $\widehat{\chi}_{\lambda}$ é a única solução do seguinte problema:

$$
\left\{\begin{array}{l}
\text { Encontrar } \widehat{\chi}_{\lambda} \in W_{\text {per }}(Y) \text { tal que } \\
a\left(\widehat{\chi}_{\lambda}, v\right)=\int_{\mathbb{R}^{n}} A \lambda \nabla v d y, \forall v \in C_{0}^{\infty}\left(\mathbb{R}^{n}\right) .
\end{array}\right.
$$

onde

$$
a\left(\widehat{\chi}_{\lambda}, v\right)=\int_{\mathbb{R}^{n}} A \nabla \widehat{\chi}_{\lambda} \nabla v d y, \forall v \in C_{0}^{\infty}\left(\mathbb{R}^{n}\right) .
$$

Para cada $\lambda \in \mathbb{R}^{n}$ definimos

$$
\widehat{w}_{\lambda}=-\widehat{\chi}_{\lambda}+\lambda \cdot y
$$

$\operatorname{com} \lambda \cdot y=\sum_{i=1}^{n} \lambda_{i} y_{i}$

Analogamente definimos para cada $\lambda \in \mathbb{R}^{n}$ a função $\chi_{\lambda}$ como sendo a única solução do problema (2.38) trocando a matriz $A$ por sua transposta $A^{t}$. Definimos $w_{\lambda}$ como sendo $-\chi_{\lambda}+\lambda \cdot y$.

Agora podemos enunciar o seguinte

Teorema 2.4.1. Seja $f \in L^{2}(\Omega)$ e $u^{\epsilon}$ solução de (2.31) com $A^{\epsilon}$ definido por (2.32)-(2.34). Então

$$
\left\{\begin{array}{l}
\text { i) } u^{\epsilon} \rightarrow u^{0} \text { em } H_{0}^{1}(\Omega) . \\
\text { ii) } A^{\epsilon} \nabla u^{\epsilon} \rightarrow A^{0} \nabla u^{0} \text { em }\left(L^{2}(\Omega)\right)^{n},
\end{array}\right.
$$

onde $u^{0}$ é a única solução em $H_{0}^{1}(\Omega)$ do problema homogeneizado

$$
\left\{\begin{array}{l}
-\sum_{i, j=1}^{n} \frac{\partial}{\partial x_{i}}\left(a_{i j}^{0} \frac{\partial u^{0}}{\partial x_{j}}\right)=f \text { em } \Omega \\
u^{0}=0 \text { em } \partial \Omega .
\end{array}\right.
$$

A matriz $A^{0}=\left(a_{i j}^{0}\right)_{1 \leq i, j \leq n}$ é constante, elíptica e dada por

$$
A^{0} \lambda=M_{Y}\left(A \nabla \widehat{w}_{\lambda}\right), \forall \lambda \in \mathbb{R}^{n}
$$


ou equivalentemente

$$
A^{0 t} \lambda=M_{Y}\left(A^{t} \nabla w_{\lambda}\right), \forall \lambda \in \mathbb{R}^{n} .
$$

Apresentamos aqui os principais passos para uma demonstração deste teorema. A ideia central é introduzir as funções $w_{\lambda}^{\epsilon}$ de modo a podermos eliminar integrais que contenham produtos de funções que apenas convergem fracamente em $L^{2}$. Definimos $w_{\lambda}^{\epsilon}$ por

$$
w_{\lambda}^{\epsilon}(x)=\epsilon w_{\lambda}\left(\frac{x}{\epsilon}\right)=\lambda \cdot x-\epsilon \chi_{\lambda}\left(\frac{x}{\epsilon}\right),
$$

onde as funções $w_{\lambda}$ e $\chi_{\lambda}$ são as definidas anteriormente.

Usando a periodicidade de $\chi_{\lambda}$ e $\nabla \chi_{\lambda}$ demonstra-se que

$$
\left\{\begin{array}{l}
\text { i) } w_{\lambda}^{\epsilon} \rightarrow \lambda \cdot x \text { em } H^{1}(\Omega) \\
\text { ii) } w_{\lambda}^{\epsilon} \rightarrow \lambda \cdot x \text { em } L^{2}(\Omega),
\end{array}\right.
$$

Definidas as funções $w_{\lambda}^{\epsilon}$, definimos $\eta_{\lambda}^{\epsilon}$ por

$$
\eta_{\lambda}^{\epsilon}(x)=\left(\left(A^{\epsilon}\right)^{t} \nabla w_{\lambda}^{\epsilon}\right)(x)=\left(A^{t} \nabla_{y} w_{\lambda}\right)\left(\frac{x}{\epsilon}\right) .
$$

Demonstra-se que

$$
\eta_{\lambda}^{\epsilon} \rightarrow M_{Y}\left(A^{t} \nabla w_{\lambda}\right)=\left(A^{0}\right)^{t} \lambda \text { em }\left(L^{2}(\Omega)\right)^{n}
$$

com $A^{0}$ definida por (2.43). Mais ainda,

$$
\int_{\Omega} \eta_{\lambda}^{\epsilon} \nabla v d x=0, \forall v \in H_{0}^{1}(\Omega)
$$

Note que $u^{\epsilon}$ satisfaz

$$
\int_{\Omega} A^{\epsilon} \nabla u^{\epsilon} \nabla v d x=\int_{\Omega} f v d x \forall v \in H_{0}^{1}(\Omega)
$$

Seja $\varphi \in C_{0}^{\infty}(\Omega)$ e escolha $\varphi w_{\lambda}^{\epsilon}$ como função teste em (2.49) e $\varphi u^{\epsilon}$ como função teste em (2.48). Então obtemos

$$
\begin{gathered}
\int_{\Omega} A^{\epsilon} \nabla u^{\epsilon} \cdot \nabla w_{\lambda}^{\epsilon} \varphi d x+\int_{\Omega} A^{\epsilon} \nabla u^{\epsilon} \cdot \nabla \varphi w_{\lambda}^{\epsilon} d x=\int_{\Omega} f \varphi w_{\lambda}^{\epsilon} d x, \forall \varphi \in C_{0}^{\infty}(\Omega), \\
\int_{\Omega} \eta_{\lambda}^{\epsilon} \cdot \nabla u^{\epsilon} \varphi d x+\int_{\Omega} \eta_{\lambda}^{\epsilon} \cdot \nabla \varphi u^{\epsilon} d x=0 . \forall \varphi \in C_{0}^{\infty}(\Omega) .
\end{gathered}
$$

Mostrando que

$$
A^{\epsilon} \nabla u^{\epsilon} \cdot \nabla w_{\lambda}^{\epsilon}=\eta_{\lambda}^{\epsilon} \cdot \nabla u^{\epsilon}
$$


obtemos por subtração

$$
\int_{\Omega} A^{\epsilon} \nabla u^{\epsilon} \cdot \nabla \varphi w_{\lambda}^{\epsilon} d x-\int_{\Omega} \eta_{\lambda}^{\epsilon} \cdot \nabla \varphi u^{\epsilon} d x=\int_{\Omega} f \varphi w_{\lambda}^{\epsilon} d x, \forall \varphi \in C_{0}^{\infty}(\Omega) .
$$

Assim obtemos uma nova equação cujos integrandos contém produto de sequências que ao menos uma é fortemente convergente. Por passagem ao limite e sabendo que $\nabla[(\lambda \cdot x) \varphi]=$ $\lambda \varphi+\lambda \cdot x \nabla \varphi$ obtemos

$$
\int_{\Omega} \xi^{0} \cdot \nabla[(\lambda \cdot x) \varphi] d x-\int_{\Omega} \xi^{0} \cdot \lambda \varphi d x-\int_{\Omega}\left(A^{0}\right)^{t} \lambda \cdot \nabla \varphi u^{0} d x=\int_{\Omega} f[(\lambda \cdot x) \varphi] d x
$$

para toda $\varphi \in C_{0}^{\infty}(\Omega)$. Como

$$
\int_{\Omega} \xi^{0} \cdot \nabla[(\lambda \cdot x) \varphi] d x=\int_{\Omega} f[(\lambda \cdot x) \varphi] d x
$$

segue que para toda $\varphi \in C_{0}^{\infty}(\Omega)$,

$$
\int_{\Omega}\left(\xi^{0} \cdot \lambda\right) \varphi d x=-\int_{\Omega}\left(A^{0}\right)^{t} \lambda \cdot \nabla \varphi u^{0} d x=\int_{\Omega}\left(\left(A^{0}\right)^{t} \lambda \cdot \nabla u^{0}\right) \varphi d x
$$

e portanto

$$
\xi^{0} \cdot \lambda=\left(A^{0}\right)^{t} \lambda \nabla u^{0}=A^{0} \nabla u^{0} \cdot \lambda
$$

Como $\lambda \in \mathbb{R}^{n}$ é arbitrário, concluímos o esboço da demonstração do resultado. 


\section{Capítulo 3}

\section{Homogeneização em domínios finos altamente oscilantes}

\subsection{Introdução}

Seja $f: \mathbb{R} \rightarrow \mathbb{R}$ uma função L-periódica e de classe $C^{1}$ com

$$
0<g_{0} \leq g(x) \leq g_{1} \forall x \in \mathbb{R}
$$

onde $g_{1}=\max \{g(x) ; x \in \mathbb{R}\}$ e $g_{0}=\min \{g(x) ; x \in \mathbb{R}\}$. Se

$$
R^{\epsilon}=\left\{(x, y) \in \mathbb{R}^{2} ; 0<x<1 \text { e } 0<y<\epsilon g(x / \epsilon)\right\}
$$

onde $\epsilon>0$ é arbitrário. O conjunto $R^{\epsilon}$ é um domínio fino que se degenera a um segmento de reta quando $\epsilon$ tende a zero. Note que $R^{\epsilon}$ tem espessura de ordem $\epsilon$, a fronteira de $R_{\epsilon}$ tem oscilações de amplitude $\epsilon$ e estas oscilaçãoes tem período de ordem $\epsilon$.

Considere o seguinte problema

$$
\left\{\begin{array}{c}
-\Delta v^{\epsilon}+v^{\epsilon}=f\left(v^{\epsilon}\right) \text { em } R^{\epsilon} \\
\frac{\partial v^{\epsilon}}{\partial \nu^{\epsilon}}=0 \text { em } \partial R^{\epsilon}
\end{array}\right.
$$

onde $\nu^{\epsilon}$ é a normal unitária exterior a $\partial R^{\epsilon}$.

Seja

$$
\Omega^{\epsilon}=\left\{\left(x_{1}, x_{2}\right) \in \mathbb{R}^{2} ; 0<x_{1}<1 \text { e } 0<x_{2}<g\left(x_{1} / \epsilon\right)\right\}
$$

e considere o difeomorfismo

$$
\varphi^{\epsilon}: \Omega^{\epsilon} \rightarrow R^{\epsilon}, \varphi\left(x_{1}, x_{2}\right)=(x, y) \text { onde } x=x_{1} \text { e } y=\epsilon x_{2}
$$


Então $v^{\epsilon}$ é solução de 3.1 se e somente se $u^{\epsilon}:=v^{\epsilon} \circ \varphi^{\epsilon}$ é solução de

$$
\left\{\begin{array}{c}
-\frac{\partial^{2} u^{\epsilon}}{\partial x_{1}^{2}}-\frac{1}{\epsilon^{2}} \frac{\partial^{2} u^{\epsilon}}{\partial x_{2}^{2}}+u^{\epsilon}=f\left(u^{\epsilon}\right) \text { em } \Omega^{\epsilon} \\
\frac{\partial u^{\epsilon}}{\partial x_{1}} N_{1}^{\epsilon}+\frac{1}{\epsilon^{2}} \frac{\partial u^{\epsilon}}{\partial x_{2}} N_{2}^{\epsilon}=0 \text { em } \partial \Omega^{\epsilon}
\end{array}\right.
$$

onde $N^{\epsilon}=\left(N_{1}^{\epsilon}, N_{2}^{\epsilon}\right)$ é o vetor normal, unitário exterior a $\partial \Omega^{\epsilon}$. Verifiquemos as condições de fronteira dos problemas (3.1) e (3.2) referentes a parte superior da fronteira.

De fato,

$$
\begin{gathered}
\nu^{\epsilon}(x, \epsilon g(x / \epsilon)):=\left(\eta_{1}^{\epsilon}, \eta_{2}^{\epsilon}\right)=\left(\frac{-g^{\prime}(x / \epsilon)}{\sqrt{1+g^{\prime}(x / \epsilon)^{2}}}, \frac{1}{\sqrt{1+g^{\prime}(x / \epsilon)^{2}}}\right) \\
N^{\epsilon}(x, g(x / \epsilon)):=\left(N_{1}^{\epsilon}, N_{2}^{\epsilon}\right)=\left(\frac{-\epsilon^{-1} g^{\prime}(x / \epsilon)}{\sqrt{1+g^{\prime}(x / \epsilon)^{2} \epsilon^{-2}}}, \frac{1}{\sqrt{1+g^{\prime}(x / \epsilon)^{2} \epsilon^{-2}}}\right)
\end{gathered}
$$

e portanto

$$
\nu^{\epsilon}=k^{\epsilon}\left(\epsilon N_{1}^{\epsilon}, N_{2}^{\epsilon}\right), k^{\epsilon}=\frac{\sqrt{1+g^{\prime}(x / \epsilon)^{2} \epsilon^{-2}}}{\sqrt{1+g^{\prime}(x / \epsilon)^{2}}} .
$$

Como $\frac{\partial u^{\epsilon}}{\partial x_{1}}=\frac{\partial v^{\epsilon}}{\partial x}$ e $\frac{\partial u^{\epsilon}}{\partial x_{2}}=\epsilon \frac{\partial v^{\epsilon}}{\partial y}$ segue que

$$
\begin{aligned}
\frac{\partial v^{\epsilon}}{\partial \nu^{\epsilon}}=0 & \Leftrightarrow\left(\frac{\partial u^{\epsilon}}{\partial x_{1}}, \frac{1}{\epsilon} \frac{\partial u^{\epsilon}}{\partial x_{2}}\right)\left(k^{\epsilon}\left(\epsilon N_{1}^{\epsilon}, N_{2}^{\epsilon}\right)\right)=0 \Leftrightarrow \frac{\partial u^{\epsilon}}{\partial x_{1}} N_{1}^{\epsilon}+\frac{1}{\epsilon} \frac{\partial u^{\epsilon}}{\partial x_{2}} N_{2}^{\epsilon}=0 \\
& \Leftrightarrow \frac{\partial u^{\epsilon}}{\partial x_{1}} N_{1}^{\epsilon}+\frac{1}{\epsilon^{2}} \frac{\partial u^{\epsilon}}{\partial x_{2}} N_{2}^{\epsilon}=0
\end{aligned}
$$

A verificação das outras equivalências é mais simples, notando apenas que

$$
\frac{\partial^{2} v^{\epsilon}}{\partial x^{2}}=\frac{\partial^{2} u^{\epsilon}}{\partial x_{1}^{2}} \text { e } \frac{\partial^{2} v^{\epsilon}}{\partial y^{2}}=\frac{1}{\epsilon^{2}} \frac{\partial^{2} u^{\epsilon}}{\partial x_{1}^{2}} .
$$

Percebe-se que o termo $\frac{1}{\epsilon^{2}}$, que acompanha a derivada segunda de $u_{\epsilon}$ com respeito a $x_{2}$, corresponde a uma difusibilidade alta na direção da variável $x_{2}$. Isto parece indicar que, no limite, a variável desconhecida $u^{\epsilon}$ deve ser independente de $x_{2}$. Por outro lado, o domínio $\Omega_{\epsilon}$ ainda depende de $\epsilon$ e possui oscilações cuja amplitude é fixa e cujo período tende a zero. Como determinar o problema limite? Veremos na seção seguinte que o método das múltiplas escalas pode ser muito útil na identificação deste problema limite. Uma vez identificado o problema limite, estudaremos a convergência compacta das soluções. 


\subsection{O Método das Múltiplas Escalas e o Problema Limite}

Nesta seção aplicamos o método das múltiplas escalas para obter, formalmente, o problema limite homogeneizado de

$$
\left\{\begin{array}{c}
-\Delta u^{\epsilon}+u^{\epsilon}=f\left(u^{\epsilon}\right) \text { em } R^{\epsilon} \\
\frac{\partial u^{\epsilon}}{\partial \nu^{\epsilon}}=0 \text { em } \partial R^{\epsilon}
\end{array}\right.
$$

sobre o domínio fino oscilante $R^{\epsilon}$ da seção anterior com $f^{\epsilon} \in L^{2}\left(R^{\epsilon}\right)$ satisfazendo $\left\|f^{\epsilon}\right\|_{L^{2}\left(R^{\epsilon}\right)} \leq$ $C$, para algum $C>0$ independente de $\epsilon$. Assumimos por simplidade que $f^{\epsilon}=f$ satisfazendo $f\left(x_{1}, x_{2}\right)=f\left(x_{1}\right)$ para todo $x_{1} \in(0,1)$ e $\epsilon>0$.

Considere a seguinte expanção formal.

$$
u^{\epsilon}\left(x_{1}, x_{2}\right)=u_{0}\left(x_{1}, x_{2}, \frac{x_{1}}{\epsilon}, \frac{x_{2}}{\epsilon}\right)+\epsilon u_{1}\left(x_{1}, x_{2}, \frac{x_{1}}{\epsilon}, \frac{x_{2}}{\epsilon}\right)+\epsilon^{2} u_{2}\left(x_{1}, x_{2}, \frac{x_{1}}{\epsilon}, \frac{x_{2}}{\epsilon}\right)+\cdots
$$

Note que o domínio fino $R^{\epsilon}$ se degenera em um segmento de reta quando $\epsilon \rightarrow 0$. Isto sugere que $u^{\epsilon}$ tende a não depender da variável "macroscópica" $x_{2}$. Assim supomos que $u_{i}$ não depende de $x_{2}$; isto é,

$$
u^{\epsilon}\left(x_{1}, x_{2}\right)=u_{0}\left(x_{1}, \frac{x_{1}}{\epsilon}, \frac{x_{2}}{\epsilon}\right)+\epsilon u_{1}\left(x_{1}, \frac{x_{1}}{\epsilon}, \frac{x_{2}}{\epsilon}\right)+\epsilon^{2} u_{0}\left(x_{1}, \frac{x_{1}}{\epsilon}, \frac{x_{2}}{\epsilon}\right)+\cdots
$$

Denotando $x=x_{1}, y=x_{1} / \epsilon, z=x_{2} / \epsilon$ obtemos

$$
\frac{\partial}{\partial x_{1}}=\partial_{x}+\frac{1}{\epsilon} \partial_{y}, \frac{\partial}{\partial x_{2}}=\frac{1}{\epsilon} \partial_{z}, \frac{\partial^{2}}{\partial x_{1}^{2}}=\partial_{x}^{2}+\frac{2}{\epsilon} \partial_{x y}^{2}+\frac{1}{\epsilon^{2}} \partial_{y}^{2}, \quad \frac{\partial^{2}}{\partial x_{2}^{2}}=\frac{1}{\epsilon^{2}} \partial_{z}^{2}
$$

Denotamos por $Y^{*}$ a seguinte célula

$$
Y^{*}=\left\{(y, z) \in \mathbb{R}^{2} ; 0<y<L \text { e } 0<z<g(y)\right\}
$$

Então

$$
\begin{aligned}
& -f^{\epsilon}=\left(-u_{0}-\epsilon u_{1}-\epsilon^{2} u_{2}-\cdots\right)+\left(\frac{\partial^{2} u_{0}}{\partial x^{2}}+\frac{2}{\epsilon} \frac{\partial^{2} u_{0}}{\partial x \partial y}+\frac{1}{\epsilon^{2}} \frac{\partial^{2} u_{0}}{\partial y^{2}}+\frac{1}{\epsilon^{2}} \frac{\partial^{2} u_{0}}{\partial z^{2}}\right) \\
& +\left(\epsilon \frac{\partial^{2} u_{1}}{\partial x^{2}}+2 \frac{\partial^{2} u_{1}}{\partial x \partial y}+\frac{1}{\epsilon} \frac{\partial^{2} u_{1}}{\partial y^{2}}+\frac{1}{\epsilon} \frac{\partial^{2} u_{1}}{\partial z^{2}}\right)+\left(\epsilon^{2} \frac{\partial^{2} u_{2}}{\partial x^{2}}+2 \epsilon \frac{\partial^{2} u_{2}}{\partial x \partial y}+\frac{\partial^{2} u_{2}}{\partial y^{2}}+\frac{\partial^{2} u_{2}}{\partial z^{2}}\right)+\cdots \\
& =\frac{1}{\epsilon^{2}} \Delta_{y, z} u_{0}+\frac{1}{\epsilon}\left(\Delta_{y, z} u_{1}+2 \frac{\partial^{2} u_{0}}{\partial x \partial y}\right)+\left(\Delta_{y, z} u_{2}+\frac{\partial^{2} u_{2}}{\partial x^{2}}+2 \frac{\partial^{2} u_{1}}{\partial x \partial y}-u_{0}\right) \\
& +\epsilon\left(\frac{\partial^{2} u_{1}}{\partial x^{2}}+2 \frac{\partial^{2} u_{2}}{\partial x \partial y}+\Delta_{y, z} u_{3}-u_{1}\right)+\cdots
\end{aligned}
$$

que motiva a analisar as seguintes equações em $Y^{*} \operatorname{com} x \in(0,1)$ fixado 


$$
\begin{aligned}
& -\Delta_{y, z} u_{0}(x, y, z)=0 \\
& -\Delta_{y, z} u_{1}(x, y, z)=2 \frac{\partial^{2} u_{0}}{\partial x \partial y}(x, y, z) \\
& -\Delta_{y, z} u_{2}(x, y, z)=f(x)-u_{0}(x, y, z)+2 \frac{\partial^{2} u_{1}}{\partial x \partial y}(x, y, z)+\frac{\partial^{2} u_{0}}{\partial x^{2}}(x, y, z) .
\end{aligned}
$$

Por outro lado, se $\eta^{\epsilon}\left(x_{1}, x_{2}\right)$ e $N\left(\frac{x_{1}}{\epsilon}, \frac{x_{2}}{\epsilon}\right)$ são normais unitários exteriores a $\partial R^{\epsilon}$ e $Y^{*}$ respectivamente, então $\eta^{\epsilon}\left(x_{1}, x_{2}\right)=N\left(\frac{x_{1}}{\epsilon}, \frac{x_{2}}{\epsilon}\right)$ desde que $x_{2}=0$ ou $x_{2}=\epsilon g\left(x_{1} / \epsilon\right)$. Se $x_{2}=0$, então $\eta^{\epsilon}\left(x_{1}, x_{2}\right)=N\left(\frac{x_{1}}{\epsilon}, \frac{x_{2}}{\epsilon}\right)=(0,-1)$. Se $x_{2}=g\left(x_{1} / \epsilon\right)$, então

$$
\eta^{\epsilon}\left(x_{1}, x_{2}\right)=\left(\frac{-g^{\prime}\left(x_{1} / \epsilon\right)}{\sqrt{1+g^{\prime}\left(x_{1} / \epsilon\right)^{2}}}, \frac{1}{\sqrt{1+g^{\prime}\left(x_{1} / \epsilon\right)^{2}}}\right)=N\left(\frac{x_{1}}{\epsilon}, \frac{x_{2}}{\epsilon}\right)
$$

pois a parte superior de $\partial R^{\epsilon}$ é da forma $\left(x_{1}, \epsilon g\left(x_{1} / \epsilon\right)\right)$ com vetor tangente $\left(1, g^{\prime}\left(x_{1} / \epsilon\right)\right)$ e a parte superior de $Y^{*}$ é da forma $(x, g(x))$ com vetor tangente $\left(1, \mathrm{~g}^{\prime}(\mathrm{x})\right)$. Assim, voltando ao problema 3.3 obtemos

$$
\begin{aligned}
0= & \nabla u^{\epsilon}\left(x_{1}, x_{2}\right) \eta^{\epsilon}\left(x_{1}, x_{2}\right) \\
= & \left(\frac{\partial u_{0}}{\partial x}+\frac{1}{\epsilon} \frac{\partial u_{0}}{\partial y}+\epsilon \frac{\partial u_{1}}{\partial x}+\frac{\partial u_{1}}{\partial y}+\epsilon^{2} \frac{\partial u_{2}}{\partial x}+\epsilon \frac{\partial u_{2}}{\partial y}+\cdots\right) \eta_{1}^{\epsilon} \\
& +\left(\frac{1}{\epsilon} \frac{\partial u_{0}}{\partial z}+\frac{\partial u_{1}}{\partial z}+\epsilon \frac{\partial u_{2}}{\partial z}+\cdots\right) \eta_{2}^{\epsilon} \\
= & \frac{1}{\partial u_{0}} \frac{x_{1}}{\partial N}\left(x_{1}, \frac{x_{2}}{\epsilon}, \frac{x_{2}}{\epsilon}\right) \\
& +\left(\frac{\partial u_{1}}{\partial N}\left(x_{1}, \frac{x_{1}}{\epsilon}, \frac{x_{2}}{\epsilon}\right)+\frac{\partial u_{0}}{\partial x}\left(x_{1}, \frac{x_{1}}{\epsilon}, \frac{x_{2}}{\epsilon}\right) N_{1}\left(\frac{x_{1}}{\epsilon}, \frac{x_{2}}{\epsilon}\right)\right) \\
& +\epsilon\left(\frac{\partial u_{2}}{\partial N}\left(x_{1}, \frac{x_{1}}{\epsilon}, \frac{x_{2}}{\epsilon}\right)+\frac{\partial u_{1}}{\partial x}\left(x_{1}, \frac{x_{1}}{\epsilon}, \frac{x_{2}}{\epsilon}\right) N_{1}\left(\frac{x_{1}}{\epsilon}, \frac{x_{2}}{\epsilon}\right)\right)+\cdots
\end{aligned}
$$

Sejam $B_{0}$ a fronteira lateral, $B_{1}$ a fronteira superior e $B_{2}$ a fronteira inferior de $Y^{*}$. Também vamos assumir que as funções $u_{i}$ são L-periódicas na variável y.

As equações (3.6) e (3.7) nos motivam a considerar o seguinte problema

$$
\left\{\begin{array}{l}
-\Delta_{y, z} u_{0}(x, y, z)=0 \text { em } Y^{*} \\
\frac{\partial u_{0}}{\partial N}=0 \text { em } B_{1} \cup B_{2} \\
u_{0}(x, \cdot, z) \text { L-periódica }
\end{array}\right.
$$


Consequentemente, obtemos $u_{0}(x, y, z)=u_{0}(x)$. Assim, podemos obter o seguinte problema

$$
\left\{\begin{array}{l}
-\Delta_{y, z} u_{1}(x, y, z)=2 \partial_{x} \partial_{y} u_{0} \text { em } Y^{*} \\
\frac{\partial u_{1}}{\partial N}(x, y, g(y))=\frac{g^{\prime}(y)}{\sqrt{1+g^{\prime}(y)^{2}}} \frac{d u_{0}}{d x} \text { em } B_{1} \\
\frac{\partial u_{1}}{\partial N}(x, y, 0)=0 \text { em } B_{2} \\
u_{1}(x, \cdot, z) \text { L-periódica }
\end{array}\right.
$$

Notando que $\partial_{x} \partial_{y} u_{0}=0$ e denotando por $X(y, z)$ a solução de

$$
\left\{\begin{array}{l}
-\Delta_{y, z} X(y, z)=0 \text { em } Y^{*} \\
\frac{\partial X}{\partial N}(y, g(y))=-\frac{g^{\prime}(y)}{\sqrt{1+g^{\prime}(y)^{2}}} \text { em } B_{1} \\
\frac{\partial X}{\partial N}(y, 0)=0 \text { em } B_{2} \\
X(\cdot, z) \text { L-periódica }
\end{array}\right.
$$

obtemos $u_{1}(x, y, z)=-X(y, z) \frac{d u_{0}}{d x}(x)$.

Analogamente, obtemos

$$
\left\{\begin{array}{l}
-\Delta_{y, z} u_{2}(x, y, z)=f(x)-u_{0}(x)+2 \partial_{x} \partial_{y} u_{1}(x, y, z)+\partial_{x}^{2} u_{0} \quad \text { em } Y^{*} \\
\frac{\partial u_{2}}{\partial N}(x, y, g(y))=-\frac{g^{\prime}(y)}{\sqrt{1+g^{\prime}(y)^{2}}} X(y, g(y)) \frac{d^{2} u_{0}}{d x^{2}} \quad \text { em } B_{1} \\
\frac{\partial u_{2}}{\partial N}(x, y, 0)=0 \quad \text { em } B_{2} \\
u_{2}(x, \cdot, z) \quad \text { L-periódica }
\end{array}\right.
$$

ou equivalentemente

$$
\left\{\begin{array}{l}
-\Delta_{y, z} u_{2}(x, y, z)=f(x)-u_{0}(x)+\left(1-2 \partial_{y} X(y, z)\right) \frac{d^{2} u_{0}}{d x^{2}}(x), \quad \text { em } Y^{*} \\
\frac{\partial u_{2}}{\partial N}(x, y, g(y))=-\frac{g^{\prime}(y)}{\sqrt{1+g^{\prime}(y)^{2}}} X(y, g(y)) \frac{d^{2} u_{0}}{d x^{2}}, \quad \text { em } B_{1} \\
\frac{\partial u_{2}}{\partial N}(x, y, 0)=0, \quad \text { em } B_{2} \\
u_{2}(x, \cdot, z), \quad \text { L-periódica }
\end{array}\right.
$$

Se $u_{2}$ é solução de (3.8), segue que

$$
0=\int_{\partial Y^{*}} \frac{\partial u_{2}}{\partial N} d S+\int_{Y^{*}}\left\{f(x)-u_{0}(x)+\left(1-2 \partial_{y} X(y, z)\right) \frac{d^{2} u_{0}}{d x^{2}}(x)\right\} d y d z
$$


Por outro lado, segue do teorema da divergência que

$$
\oint_{\gamma} \vec{F} \cdot \vec{n} d s=\int_{Y^{*}} \operatorname{div} \vec{F} d y d z
$$

onde $\vec{F}=(X, 1)$ e $\gamma$ é uma parametrização $C^{1}$ por partes de $\partial Y^{*}$ orientada no sentido antihorário. Sabendo-se que

$$
\oint_{\gamma} \vec{F} \cdot \vec{n} d s=\int_{I} \vec{F}(\gamma(t)) \cdot \vec{n}(\gamma(t))\left\|\gamma^{\prime}(t)\right\| d t
$$

e usando que $X$ é L-periódica em $y$ obtemos

$$
\oint_{\gamma} \vec{F} \cdot \vec{n} d s=\int_{0}^{L} X(t, g(t)) g^{\prime}(t) d t
$$

Logo,

$$
\int_{Y^{*}} \partial_{y} X(y, z) d y d z=\int_{Y^{*}} \operatorname{div} \vec{F} d y d z=\int_{0}^{L} X(t, g(t)) g^{\prime}(t) d t
$$

Por outro lado, do fato que as derivadas de $u_{2}$ são L-periódicas em y e das condições de contorno satisfeitas por $u_{2}$ segue que

$$
\begin{aligned}
\int_{\partial Y^{*}} \frac{\partial u_{2}}{\partial N} d S & =\int_{I} \frac{\partial u_{2}}{\partial N}(\gamma(t))\left\|\gamma^{\prime}(t)\right\| d t \\
& =-\int_{0}^{L} \frac{\partial u_{2}}{\partial N}(\gamma(t)) \sqrt{1+g^{\prime}(t)^{2}} d t \\
& =\frac{d^{2} u_{0}}{d x^{2}} \int_{0}^{L} g^{\prime}(t) X(t, g(t)) d t
\end{aligned}
$$

$\log 0$

$$
\int_{\partial Y^{*}} \frac{\partial u_{2}}{\partial N} d S=\frac{d^{2} u_{0}}{d x^{2}} \int_{Y^{*}} \partial_{y} X(y, z) d y d z
$$

Voltando a equação (3.9), obtemos

$$
\int_{Y^{*}}\left\{f(x)-u_{0}(x)+\left(1-\partial_{y} X(y, z)\right) \frac{d^{2} u_{0}}{d x^{2}}\right\} d y d z=0
$$

Como $X=X(y, z)$ podemos concluir que $u_{0}$ deve satisfazer

$$
\left\{\begin{array}{c}
-q_{0} \frac{d^{2} u_{0}}{d x^{2}}(x)+u_{0}(x)=f(x), x \in(0,1) \\
u_{0}^{\prime}(0)=u_{0}^{\prime}(1)=0
\end{array}\right.
$$

onde

$$
q_{0}=\frac{1}{\left|Y^{*}\right|} \int_{Y^{*}}\left\{1-\frac{\partial X}{\partial y}(y, z)\right\} d y d z
$$

A equação diferencial acima em $u_{0}$ definida no intervalo $(0,1)$ é chamada de equação homogeneizada do problema (3.3). 


\subsection{O Operador Extensão}

Nesta seção construímos um operador extensão que será fundamental para se tratar do problema de convergência das soluções de (3.3) para as soluções de (3.15), permitindo que provemos a convergência compacta de resolventes nas seções subsequentes.

Considere os seguintes conjuntos abertos

$$
\begin{gathered}
\mathcal{O}=\left\{\left(x_{1}, x_{2}\right) \in \mathbb{R}^{2} ; x_{1} \in I \text { e } 0<x_{2}<G_{1}\right\} \\
\mathcal{O}^{\epsilon}=\left\{\left(x_{1}, x_{2}\right) \in \mathbb{R}^{2} ; x_{1} \in I \text { e } 0<x_{2}<G_{\epsilon}\left(x_{1}\right)\right\}
\end{gathered}
$$

onde $I \subset \mathbb{R}$ é um intervalo aberto limitado, $G_{\epsilon}: I \mapsto \mathbb{R}$ é uma função $C^{1}$ satisfazendo $0<G_{0} \leq G_{\epsilon}(x) \leq G_{1}$ para todo $x$ em $I$ e $\epsilon>0$. Note que $\mathcal{O}^{\epsilon} \subset \mathcal{O}$

Lema 3.3.1. Com a mesma notação acima, existe um operador extensão

$$
P_{\epsilon} \in \mathcal{L}\left(L^{p}\left(\mathcal{O}^{\epsilon}\right), L^{p}(\mathcal{O})\right) \cap \mathcal{L}\left(W^{1, p}\left(\mathcal{O}^{\epsilon}\right), W^{1, p}(\mathcal{O})\right) \cap \mathcal{L}\left(W_{\partial_{l}}^{1, p}\left(\mathcal{O}^{\epsilon}\right), W_{\partial_{l}}^{1, p}(\mathcal{O})\right)
$$

(onde $W_{\partial_{l}}^{1, p}(\mathcal{O})\left(W_{\partial_{l}}^{1, p}\left(\mathcal{O}^{\epsilon}\right)\right)$ é o conjunto das funções em $W^{1, p}(\mathcal{O})\left(W^{1, p}\left(\mathcal{O}^{\epsilon}\right)\right)$ que se anulam na fronteira vertical de $\mathcal{O}\left(\mathcal{O}^{\epsilon}\right)$ ) e uma constante $K$ independente de $\epsilon$ e $p$ tais que

$$
\begin{aligned}
\left\|P_{\epsilon} \varphi\right\|_{L^{p}(\mathcal{O})} & \leq K\|\varphi\|_{L^{p}\left(\mathcal{O}^{\epsilon}\right)} \\
\left\|\frac{\partial P_{\epsilon} \varphi}{\partial x_{1}}\right\|_{L^{p}(\mathcal{O})} & \leq K\left\{\left\|\frac{\partial \varphi}{\partial x_{1}}\right\|_{L^{p}\left(\mathcal{O}^{\epsilon}\right)}+\eta(\epsilon)\left\|\frac{\partial \varphi}{\partial x_{2}}\right\|_{L^{p}\left(\mathcal{O}^{\epsilon}\right)}\right\} \\
\left\|\frac{\partial P_{\epsilon} \varphi}{\partial x_{2}}\right\|_{L^{p}(\mathcal{O})} & \leq K\left\|\frac{\partial \varphi}{\partial x_{2}}\right\|_{L^{p}\left(\mathcal{O}^{\epsilon}\right)}
\end{aligned}
$$

para toda $\varphi \in W^{1, p}\left(\mathcal{O}^{\epsilon}\right)$ onde $1 \leq p \leq \infty e$

$$
\eta(\epsilon)=\sup _{x \in I}\left\{\left|G_{\epsilon}^{\prime}(x)\right|\right\} .
$$

Prova: $\quad$ Note que o conjunto $\mathcal{O}^{0}=I \times\left(0, G_{0}\right) \subset \mathcal{O}^{\epsilon}$ para todo $\epsilon$. Dividiremos a prova em dois casos.

$1^{\circ}$ Caso. Suponha que $G_{1} \leq 2 G_{0}$, o que implica que $G_{\epsilon}(x) \leq 2 G_{0}$. Então podemos definir o operador:

$$
\left(P_{\epsilon} \varphi\right)= \begin{cases}\varphi\left(x_{1}, x_{2}\right) & \left(x_{1}, x_{2}\right) \in \mathcal{O}^{\epsilon} \\ \varphi\left(x_{1}, 2 G_{\epsilon}\left(x_{1}\right)-x_{2}\right) & \left(x_{1}, x_{2}\right) \in \mathcal{O} \backslash \mathcal{O}^{\epsilon}\end{cases}
$$

Verifiquemos que $P_{\epsilon}$ satisfaz o lema. Para isto, considere a seguinte transformação:

$$
T_{\epsilon}: \mathcal{O} \backslash \mathcal{O}^{\epsilon} \rightarrow \mathcal{O}^{\epsilon} ; T_{\epsilon}\left(x_{1}, x_{2}\right)=\left(x_{1}, 2 G_{\epsilon}\left(x_{1}\right)-x_{2}\right) .
$$


(i) Se $\varphi \in L^{p}\left(\mathcal{O}^{\epsilon}\right)$, então $\int_{\mathcal{O}^{\epsilon}}|\varphi|^{p}<\infty$ e portanto segue do teorema de mudânça de variáveis aplicado a $T_{\epsilon}$ que

$$
\int_{\mathcal{O}}\left|P_{\epsilon} \varphi\right|^{p}=\int_{\mathcal{O}^{\epsilon}}|\varphi|^{p}+\int_{\mathcal{O} \backslash \mathcal{O}^{\epsilon}}\left|P_{\epsilon} \varphi\right|^{p} \leq 2 \int_{\mathcal{O}^{\epsilon}}|\varphi|^{p}<\infty .
$$

$\operatorname{Logo} P_{\epsilon} \varphi \in L^{p}(\mathcal{O})$ e

$$
\left\|P_{\epsilon} \varphi\right\|_{L^{p}(\mathcal{O})} \leq 2\|\varphi\|_{L^{p}\left(\mathcal{O}^{\epsilon}\right)}
$$

(ii) Seja $\varphi \in W^{1, p}\left(\mathcal{O}^{\epsilon}\right)$. Mostremos que $P_{\epsilon} \varphi \in W^{1, p}(\mathcal{O})$. Seja $\phi \in C_{0}^{1}(\mathcal{O})$. Usando a Fórmula de Green 2.3.3 e a regra da cadêia obtemos

$$
\begin{aligned}
& \int_{\mathcal{O}} P_{\epsilon} \varphi \frac{\partial \phi}{\partial x_{1}} d x_{1} d x_{2}=\int_{\mathcal{O} \backslash \mathcal{O}^{\epsilon}}\left(\varphi \circ T_{\epsilon}\right) \frac{\partial \phi}{\partial x_{1}} d x_{1} d x_{2}+\int_{\mathcal{O}^{\epsilon}} \varphi \frac{\partial \phi}{\partial x_{1}} d x_{1} d x_{2} \\
& =-\int_{\mathcal{O} \backslash \mathcal{O}^{\epsilon}} \frac{\partial}{\partial x_{1}}\left(\varphi \circ T_{\epsilon}\right) \phi d x_{1} d x_{2}-\int_{\mathcal{O}^{\epsilon}} \frac{\partial \varphi}{\partial x_{1}} \phi d x_{1} d x_{2}+\int_{\partial\left(\mathcal{O} \backslash \mathcal{O}^{\epsilon}\right)}\left(\varphi \circ T_{\epsilon}\right) \phi d s+\int_{\partial \mathcal{O}^{\epsilon}} \varphi \phi d s \\
& =-\int_{\mathcal{O} \backslash \mathcal{O}^{\epsilon}}\left\{\frac{\partial \varphi}{\partial x_{1}}\left(T_{\epsilon}\left(x_{1}, x_{2}\right)\right)+2 G_{\epsilon}^{\prime}\left(x_{1}\right) \frac{\partial \varphi}{\partial x_{2}}\left(T_{\epsilon}\left(x_{1}, x_{2}\right)\right)\right\} \phi\left(x_{1}, x_{2}\right) d x_{1} d x_{2}-\int_{\mathcal{O}^{\epsilon}} \frac{\partial \varphi}{\partial x_{1}} \phi d x_{1} d x_{2} \\
& =-\int_{\mathcal{O}}\left\{P_{\epsilon}\left(\frac{\partial \varphi}{\partial x_{1}}\right)\left(x_{1}, x_{2}\right)+2 G_{\epsilon}^{\prime}\left(x_{1}\right) \chi_{\mathcal{O}^{\prime} \mathcal{O}^{\epsilon}}\left(x_{1}, x_{2}\right) P_{\epsilon}\left(\frac{\partial \varphi}{\partial x_{2}}\right)\left(x_{1}, x_{2}\right)\right\} \phi\left(x_{1}, x_{2}\right) d x_{1} d x_{2} .
\end{aligned}
$$

portanto segue que

$$
\frac{\partial P_{\epsilon} \varphi}{\partial x_{1}}\left(x_{1}, x_{2}\right)=P_{\epsilon}\left(\frac{\partial \varphi}{\partial x_{1}}\right)\left(x_{1}, x_{2}\right)+2 G_{\epsilon}^{\prime}\left(x_{1}\right) \chi_{\mathcal{O} \backslash \mathcal{O}}\left(x_{1}, x_{2}\right) P_{\epsilon}\left(\frac{\partial \varphi}{\partial x_{2}}\right)\left(x_{1}, x_{2}\right) \text { q.s em } \mathcal{O} ;
$$

e

$$
\left\|\frac{\partial P_{\epsilon} \varphi}{\partial x_{1}}\right\|_{L^{p}(\mathcal{O})} \leq 4\left(\left\|\frac{\partial \varphi}{\partial x_{1}}\right\|_{L^{p}\left(\mathcal{O}^{\epsilon}\right)}+\eta(\epsilon)\left\|\frac{\partial \varphi}{\partial x_{2}}\right\|_{L^{p}\left(\mathcal{O}^{\epsilon}\right)}\right)
$$

Analogamente,

$$
\begin{aligned}
& \int_{\mathcal{O}} P_{\epsilon} \varphi \frac{\partial \phi}{\partial x_{2}} d x_{1} d x_{2}=-\int_{\mathcal{O} \backslash \mathcal{O}^{\epsilon}} \frac{\partial}{\partial x_{2}}\left(\varphi \circ T_{\epsilon}\right) \phi d x_{1} d x_{2}-\int_{\mathcal{O}^{\epsilon}} \frac{\partial \varphi}{\partial x_{2}} \phi d x_{1} d x_{2} \\
& =-\int_{\mathcal{O} \mathcal{O}^{\epsilon}}\left[\frac{\partial \varphi}{\partial x_{1}}\left(T_{\epsilon}\left(x_{1}, x_{2}\right)\right) \cdot 0+\frac{\partial \varphi}{\partial x_{2}}\left(T_{\epsilon}\left(x_{1}, x_{2}\right)\right)(-1)\right] \phi\left(x_{1}, x_{2}\right) d x_{1} d x_{2} \\
& =-\int_{\mathcal{O}}\left[-\chi_{\mathcal{O} \backslash \mathcal{O}^{\epsilon}} P_{\epsilon}\left(\frac{\partial \varphi}{\partial x_{2}}\right)+\chi_{\mathcal{O}^{\epsilon}} P_{\epsilon}\left(\frac{\partial \varphi}{\partial x_{2}}\right)\right] \phi d x_{1} d x_{2} .
\end{aligned}
$$

e portanto segue que

e

$$
\frac{\partial P_{\epsilon} \varphi}{\partial x_{2}}=\left(\chi_{\mathcal{O}^{\epsilon}}-\chi_{\mathcal{O} \backslash \mathcal{O}^{\epsilon}}\right) P_{\epsilon}\left(\frac{\partial \phi}{\partial x_{2}}\right)
$$

$$
\left\|\frac{\partial P_{\epsilon} \varphi}{\partial x_{2}}\right\|_{L^{p}(\mathcal{O})} \leq 2\left\|\frac{\partial \varphi}{\partial x_{2}}\right\|_{L^{p}\left(\mathcal{O}^{\epsilon}\right)}
$$


Com estas contas, concluimos o $1^{\circ}$ caso tomando $K=4$. Note que do item $(i i)$ e da construção (por reflexão) de $P_{\epsilon}$ segue que $P_{\epsilon} \in \mathcal{L}\left(W_{\partial_{l}}^{1, p}\left(\mathcal{O}^{\epsilon}\right), W_{\partial_{l}}^{1, p}(\mathcal{O})\right.$ ).

$2^{\mathbf{o}}$ Caso. Suponha $G_{1}>2 G_{0}$. Neste caso faremos extensões na direção negativa de $x_{2}$ um certo número de vezes para então aplicarmos o primeiro caso.

Sejam $\mathcal{O}_{n}^{\epsilon}=\mathcal{O}^{\epsilon} \cup I \times\left[-n G_{0}, 0\right], n \in \mathbb{N}$ e $\varphi_{0} \in L^{p}\left(\mathcal{O}^{\epsilon}\right)$. Primeiro estendemos $\varphi_{0}$ para o conjunto $I \times\left(-G_{0}, 0\right)$ da seguinte forma

$$
\varphi_{1}\left(x_{1}, x_{2}\right)= \begin{cases}\varphi_{0}\left(x_{1}, x_{2}\right), & 0<x_{2}<G_{\epsilon}\left(x_{1}\right) \\ \varphi_{0}\left(x_{1},-x_{2}\right), & -G_{0}<x_{2} \leq 0\end{cases}
$$

Podemos continuar produzindo estas reflexões indutivamente como segue

$$
\varphi_{n}\left(x_{1}, x_{2}\right)= \begin{cases}\varphi_{n-1}\left(x_{1}, x_{2}\right), & -(n-1) G_{0}<x_{2}<G_{\epsilon}\left(x_{1}\right) \\ \varphi_{n-1}\left(x_{1},-x_{2}-2(n-1) G_{0}\right), & -n G_{0}<x_{2} \leq-(n-1) G_{0} .\end{cases}
$$

Escolha $n \in \mathbb{N}$ tal que $n G_{0}>G_{1}$. Definimos $\widetilde{P}_{\epsilon} \varphi_{0}:=\overline{P_{\epsilon}} \varphi_{n}$ em que $\overline{P_{\epsilon}}$ é definida como no $1^{\mathrm{o}}$ caso, só que em $\mathcal{O}_{n}^{\epsilon}$. Seja $\widetilde{\mathcal{O}}=I \times\left[-n G_{0}, G_{1}\right]$. Para cada $\varphi_{0} \in L^{p}\left(\mathcal{O}^{\epsilon}\right)$ temos $\varphi_{n} \in L^{p}\left(\mathcal{O}_{n}^{\epsilon}\right)$ $\mathrm{e}$

$$
\left\|\widetilde{P}_{\epsilon} \varphi_{0}\right\|_{L^{p}(\widetilde{\mathcal{O}})} \leq 2\left\|\varphi_{n}\right\|_{L^{p}\left(\mathcal{O}_{n}^{\epsilon}\right)} \leq 2(n+1)\left\|\varphi_{0}\right\|_{L^{p}\left(\mathcal{O}^{\epsilon}\right)}
$$

Se $\varphi_{0} \in W^{1, p}\left(\mathcal{O}^{\epsilon}\right)$, então $\widetilde{P}_{\epsilon} \varphi_{0} \in W^{1, p}\left(\mathcal{O}_{n}^{\epsilon}\right)$ e

$$
\left\|\frac{\partial \widetilde{P}_{\epsilon} \varphi_{0}}{\partial x_{2}}\right\|_{L^{p}(\widetilde{\mathcal{O}})} \leq 2\left\|\frac{\partial \varphi_{n}}{\partial x_{2}}\right\|_{L^{p}\left(\mathcal{O}_{n}^{\epsilon}\right)} \leq 2(n+1)\left\|\frac{\partial \varphi_{0}}{\partial x_{2}}\right\|_{L^{p}\left(\mathcal{O}^{\epsilon}\right)}
$$

e

$$
\begin{aligned}
\left\|\frac{\partial \widetilde{P}_{\epsilon} \varphi_{0}}{\partial x_{1}}\right\|_{L^{p}(\widetilde{\mathcal{O}})} & \leq 4\left\{\left\|\frac{\partial \varphi_{n}}{\partial x_{1}}\right\|_{L^{p}\left(\mathcal{O}_{n}^{\epsilon}\right)}+\eta(\epsilon)\left\|\frac{\partial \varphi_{n}}{\partial x_{2}}\right\|_{L^{p}\left(\mathcal{O}_{n}^{\epsilon}\right)}\right\} \\
& \leq 4(n+1)\left\{\left\|\frac{\partial \varphi_{0}}{\partial x_{1}}\right\|_{L^{p}\left(\mathcal{O}^{\epsilon}\right)}+\eta(\epsilon)\left\|\frac{\partial \varphi_{0}}{\partial x_{2}}\right\|_{L^{p}\left(\mathcal{O}^{\epsilon}\right)}\right\}
\end{aligned}
$$

Tomando $P_{\epsilon} \varphi=\left.\left(\widetilde{P}_{\epsilon} \varphi\right)\right|_{\mathcal{O}}$ obtemos um operador que satisfaz o lema. 


\subsection{Teorema de Convergência para o Limite Homogeneizado}

Nesta seção estudaremos a convergência das soluções do problema (3.2) com um potencial $V^{\epsilon}$, isto é, estudaremos a convergência das soluções do problema

$$
\left\{\begin{array}{c}
-\frac{\partial^{2} u^{\epsilon}}{\partial x_{1}^{2}}-\frac{1}{\epsilon^{2}} \frac{\partial^{2} u^{\epsilon}}{\partial x_{2}^{2}}+V^{\epsilon} u^{\epsilon}=f^{\epsilon} \text { em } \Omega^{\epsilon} \\
\frac{\partial u^{\epsilon}}{\partial x_{1}} N_{1}^{\epsilon}+\frac{1}{\epsilon^{2}} \frac{\partial u^{\epsilon}}{\partial x_{2}} N_{2}^{\epsilon}=0 \text { em } \partial \Omega^{\epsilon}
\end{array}\right.
$$

O domínio oscilante $\Omega^{\epsilon}$ e o domínio fixo $\Omega$ são dados por

$$
\Omega^{\epsilon}=\left\{\left(x_{1}, x_{2}\right) \in \mathbb{R}^{2} ; x_{1} \in I \text { e } 0<x_{2}<g\left(x_{1} / \epsilon\right)\right\}
$$

e

$$
\Omega=\left\{\left(x_{1}, x_{2}\right) \in \mathbb{R}^{2} ; x_{1} \in I \text { e } 0<x_{2}<g_{1}\right\}
$$

com $I=(0,1)$. É claro que $\Omega^{\epsilon} \subset \Omega$.

Assumimos as seguintes propriedades:

- Para todo $\epsilon \in[0,1], V^{\epsilon} \in L^{\infty}\left(\Omega^{\epsilon}\right), V^{\epsilon} \geq 1$, e $V_{0}$ é independente da variável $x_{2}$; isto é, $V_{0}\left(x_{1}, x_{2}\right)=V_{0}\left(x_{1}\right)$ tal que

$$
\sup _{\epsilon \in[0,1]}\left\|V^{\epsilon}\right\|_{L^{\infty}\left(\Omega^{\epsilon}\right)}<\infty, \quad\left\|V^{\epsilon}-V_{0}\right\|_{L^{1}\left(\Omega^{\epsilon}\right)} \rightarrow 0 .
$$

- Existe uma constante $C>0$ tal que $\left\|f^{\epsilon}\right\|_{L^{2}\left(\Omega^{\epsilon}\right)} \leq C$ para todo $\epsilon \in(0,1]$.

A formulação variacional do problema de valor de fronteira (3.19) é o seguinte problema: Encontrar $u^{\epsilon} \in H^{1}(\Omega)$ tal que

$$
\int_{\Omega^{\epsilon}}\left\{\frac{\partial u^{\epsilon}}{\partial x_{1}} \frac{\partial \varphi}{\partial x_{1}}+\frac{1}{\epsilon^{2}} \frac{\partial u^{\epsilon}}{\partial x_{2}} \frac{\partial \varphi}{\partial x_{2}}+V^{\epsilon} u^{\epsilon} \varphi\right\} d x_{1} d x_{2}=\int_{\Omega^{\epsilon}} f^{\epsilon} \varphi d x_{1} d x_{2}, \forall \varphi \in H^{1}\left(\Omega^{\epsilon}\right) .
$$

O problema (3.21) tem uma única solução pelo teorema de Lax-Milgram. Note que o lado direito de (3.21) define uma forma bilinear, contínua e coerciva sobre $H^{1}\left(\Omega^{\epsilon}\right)$.

Como $V^{\epsilon} \geq 1$, tomando $\varphi=u^{\epsilon}$ concluímos que

$$
\left\|\frac{\partial u^{\epsilon}}{\partial x_{1}}\right\|_{L^{2}\left(\Omega^{\epsilon}\right)}^{2}+\frac{1}{\epsilon^{2}}\left\|\frac{\partial u^{\epsilon}}{\partial x_{2}}\right\|_{L^{2}\left(\Omega^{\epsilon}\right)}^{2}+\left\|u^{\epsilon}\right\|_{L^{2}\left(\Omega^{\epsilon}\right)}^{2} \leq\left\|f^{\epsilon}\right\|_{L^{2}\left(\Omega^{\epsilon}\right)}\left\|u^{\epsilon}\right\|_{L^{2}\left(\Omega^{\epsilon}\right)} .
$$


Note que de (3.22) segue que $\left\{u^{\epsilon}\right\}_{0<\epsilon \leq 1}$ é limitada e portanto a sequência $\left\{P_{\epsilon} u^{\epsilon}\right\}_{0<\epsilon \leq 1}$, $P_{\epsilon} u^{\epsilon} \in H^{1}(\Omega)$ e $P_{\epsilon}$ dado pelo Lema 3.3.1, tem subsequência convergênte fracamente convergênte em $H^{1}(\Omega)$ e portanto, fortemente convergênte em $L^{2}(\Omega)$. Esta convergência será muito útil para analizarmos o comportamento limite de (3.21).

Note que ao tentarmos avaliar a equação (3.21), temos certa dificuldade em determinar a equação limite pois o domínio de integração varia com o parâmetro $\epsilon$. Mesmo estendendo por zero as derivadas de $u^{\epsilon}$ e colocando funções-teste com derivada com relação a $x_{2}$ nula, não conseguimos obter diretamente o limite da extensão por zero de $\frac{\partial u^{\epsilon}}{\partial x_{1}}$.

No que segue, tentaremos transformar a equação (3.21) numa equação com domínio fixo, usando as extensões $P_{\epsilon}$ e extensões por zero. Antes disto, apresentaremos as funções-teste oscilantes de Tartar. Estas funções, quando inseridas na equação (3.21), 'removem' a componente com o coeficiente $\frac{1}{\epsilon^{2}}$ independente de terem a derivada com relação a segunda variável sendo nula, além de auxiliarem na determinação do limite da extensão por zero de $\frac{\partial u^{\epsilon}}{\partial x_{1}}$. Inseridas estas funções-teste em (3.21) e em (3.30) (dada pelo Lema 3.4.1), estenderemos estas equações para $\Omega$ e então calcularemos o limite de cada componente das integrais. Calculados os limites das funções envolvidas, tentaremos igualar as duas equações resultantes (utilizando funções-teste 'convenientes') para então chegarmos a equação limite (3.62).

\subsubsection{Funções auxiliares periódicas}

Nesta subseção apresentamos as funções oscilantes que faremos uso para contornarmos a dificuldade de avaliar o limite da integral do termo com coeficiente $\frac{1}{\epsilon^{2}}$ em (3.22).

Considere a faixa

$$
\Sigma=\left\{\left(y_{1}, y_{2}\right) \in \mathbb{R}^{2} ; y_{1} \in \mathbb{R} \text { e } 0<y_{2}<g\left(y_{1}\right)\right\}
$$

e seja $\chi$ a sua função característica.

Se denotarmos por $\chi^{\epsilon}$ a função característica de $\Omega^{\epsilon}$, verifica-se que

$$
\chi^{\epsilon}\left(x_{1}, x_{2}\right)=\chi\left(\frac{x_{1}}{\epsilon}, x_{2}\right), \forall\left(x_{1}, x_{2}\right) \in \Omega^{\epsilon} .
$$

Consideremos a seguinte família de bijeções $T_{k}^{\epsilon}: A_{k}^{\epsilon} \mapsto Y$ dada por

$$
T_{k}^{\epsilon}\left(x_{1}, x_{2}\right)=\left(\frac{x_{1}-\epsilon k L}{\epsilon}, x_{2}\right)
$$

onde

$$
\begin{gathered}
A_{k}^{\epsilon}=\left\{\left(x_{1}, x_{2}\right) \in \mathbb{R}^{2} ; \epsilon k L<x_{1}<\epsilon(k+1) L \text { e } 0<x_{2}<g_{1}\right\} \\
Y=(0, L) \times\left(0, g_{1}\right)
\end{gathered}
$$


com $k \in \mathbb{N}$ satisfazendo $0 \leq k<1 /(\epsilon L)$.

\section{A função $X$}

A seguir, definimos a função auxiliar $X$ através do seguinte problema:

$$
\left\{\begin{array}{l}
\Delta X=0 \text { em } Y^{*} \\
\frac{\partial X}{\partial N}=0 \text { em } B_{2} \\
\frac{\partial X}{\partial N}=-\frac{g^{\prime}\left(y_{1}\right)}{\sqrt{1+g^{\prime}\left(y_{1}\right)^{2}}} \text { em } B_{1} \\
X \text { L-periódica em } y_{1} \\
M_{Y^{*}} X=0
\end{array}\right.
$$

onde $B_{1}$ é a fronteira superior e $B_{2}$ é a fronteira inferior de $\partial Y^{*}$ e

$$
Y^{*}=\left\{\left(y_{1}, y_{2}\right) \in \mathbb{R}^{2} ; 0<y_{1}<L \text { e } 0<y_{2}<g\left(y_{1}\right)\right\} .
$$

Considere a seguinte formulação variacional: encontrar $X \in H^{1}\left(Y^{*}\right), X$ L-periódica em $y_{1}$ tal que

$$
a_{Y^{*}}(X, \varphi):=\int_{Y^{*}} \nabla X \cdot \nabla \varphi d y_{1} d y_{2}=\int_{\partial Y^{*}} \frac{\partial X}{\partial N} \varphi d s=\int_{0}^{L} \varphi(y, g(y)) g^{\prime}(y) d y,
$$

para toda $\varphi \in H^{1}\left(Y^{*}\right)$ L-periódica em $y_{1}, M_{Y^{*}} \varphi=0$.

Segue do Teorema de Lax-Milgram que existe uma única solução do problema acima. Denotaremos por $X$ esta solução. Note que a coercividade de $a_{Y^{*}}$ segue da Desigualdade de Poincaré-Wirtinger (Proposição 2.3.2).

\section{As funções-teste oscilantes de Tartar}

A seguir, definimos as funções $\omega^{\epsilon}, \eta_{1}^{\epsilon}$ e $\eta_{2}^{\epsilon}$. Para isto, tomamos as bijeções (3.24) e o operador extensão

$$
P \in \mathcal{L}\left(H^{1}\left(Y^{*}\right), H^{1}(Y)\right) \cap \mathcal{L}\left(L^{2}\left(Y^{*}\right), L^{2}(Y)\right)
$$

definido no Lema 3.3.1 com $G_{\epsilon}\left(x_{1}\right)=g\left(x_{1}\right)$ independente de $\epsilon$ e $Y=(0, L) \times\left(0, g_{1}\right)$.

Definimos $\omega_{k}^{\epsilon}$ em $\left(x_{1}, x_{2}\right) \in A_{k}^{\epsilon}$ por

$$
\omega_{k}^{\epsilon}\left(x_{1}, x_{2}\right)=x_{1}-\epsilon\left(P X \circ T_{k}^{\epsilon}\left(x_{1}, x_{2}\right)\right)=x_{1}-\epsilon\left(P X\left(\frac{x_{1}-\epsilon k L}{\epsilon}, x_{2}\right) .\right)
$$


Note que para todo $\left(x_{1}, x_{2}\right)$ em $\Omega^{\epsilon}$ existe $k$ tal que $\left(x_{1}, x_{2}\right) \in A_{k}^{\epsilon}$. Portanto, a função $\omega^{\epsilon}\left(x_{1}, x_{2}\right)=\omega_{k}^{\epsilon}\left(x_{1}, x_{2}\right)$ está bem definida. Aplicando a Fórmula de Green 2.3.3 e notando que $\omega_{k}^{\epsilon}\left(\epsilon L(k+1), x_{2}\right)=\omega_{k+1}^{\epsilon}\left(\epsilon L(k+1), x_{2}\right), \forall x_{2} \in\left(0, g_{1}\right)$ obtemos $\omega^{\epsilon} \in H^{1}(\Omega)$.

Introduzimos agora o vetor $\eta^{\epsilon}=\left(\eta_{1}^{\epsilon}, \eta_{2}^{\epsilon}\right)$ definido por

$$
\eta_{i}^{\epsilon}\left(x_{1}, x_{2}\right)=\frac{\partial \omega^{\epsilon}}{\partial x_{i}}\left(x_{1}, x_{2}\right),\left(x_{1}, x_{2}\right) \in \Omega^{\epsilon}
$$

Como $\frac{\partial}{\partial x_{1}}=\frac{1}{\epsilon} \frac{\partial}{\partial y_{1}}$ e $\frac{\partial}{\partial x_{2}}=\frac{\partial}{\partial y_{2}}$, tomando a extensão periódica de $X$ na direção $y_{1}$ obtemos

$$
\begin{aligned}
& \eta_{1}^{\epsilon}\left(x_{1}, x_{2}\right)=1-\frac{\partial X}{\partial y_{1}}\left(\frac{x_{1}-\epsilon k L}{\epsilon}, x_{2}\right)=1-\frac{\partial X}{\partial y_{1}}\left(\frac{x_{1}}{\epsilon}, x_{2}\right):=\eta_{1}\left(y_{1}, y_{2}\right), \\
& \eta_{2}^{\epsilon}\left(x_{1}, x_{2}\right)=-\epsilon \frac{\partial X}{\partial y_{2}}\left(\frac{x_{1}-\epsilon k L}{\epsilon}, x_{2}\right)=-\epsilon \frac{\partial X}{\partial y_{2}}\left(\frac{x_{1}}{\epsilon}, x_{2}\right):=\eta_{2}\left(y_{1}, y_{2}\right)
\end{aligned}
$$

para $\left(y_{1}, y_{2}\right)=\left(\frac{x_{1}-\epsilon k L}{\epsilon}, x_{2}\right) \in Y^{*}$ e $\left(x_{1}, x_{2}\right) \in \Omega^{\epsilon}$. Note que $\eta_{i}$ é uma função periódica na variável $y_{1}$ e $\eta_{i}^{\epsilon}\left(x_{1}, x_{2}\right)=\eta^{\epsilon}\left(\frac{x_{1}}{\epsilon}, x_{2}\right)$ para $i=1,2$.

O seguinte lema tem um papel fundamental para o objetivo descrito no início desta subseção.

Lema 3.4.1. Dada uma função $\varphi \in H^{1}(\Omega) \operatorname{com} \varphi=0$ em uma vizinhança das fronteiras laterais existe $\epsilon_{0}>0$ tal que $\forall 0<\epsilon<\epsilon_{0}$ temos

$$
\int_{\Omega^{\epsilon}}\left(\eta_{1}^{\epsilon} \frac{\partial \varphi}{\partial x_{1}}+\eta_{2}^{\epsilon} \frac{1}{\epsilon^{2}} \frac{\partial \varphi}{\partial x_{2}}\right) d x_{1} d x_{2}=0 .
$$

Prova: De fato, seja $\epsilon>0$ tal que $\left.\varphi\right|_{A_{k_{0}}^{\epsilon} \cap \Omega} \equiv 0$ onde $k_{0}$ é o maior inteiro tal que $0 \leq k<$ $(\epsilon L)^{-1}$.

Analisemos a integral acima em cada subconjunto $A_{k}^{\epsilon} \cap \Omega^{\epsilon}, 0 \leq k<k_{0}$

Com esta restrição sobre $k$ temos os seguintes difeomorfismos

$$
T_{k}^{\epsilon}: A_{k}^{\epsilon} \cap \Omega^{\epsilon} \rightarrow Y^{*}, \psi_{k}^{\epsilon}:=\left(T_{k}^{\epsilon}\right)^{-1}: Y^{*} \rightarrow A_{k}^{\epsilon} \cap \Omega^{\epsilon}
$$

De (3.29) basta mostrarmos que

$$
\begin{gathered}
\int_{\Omega^{\epsilon}} \nabla X\left(T_{k}^{\epsilon}\left(x_{1}, x_{2}\right)\right)\left(\frac{\partial \varphi}{\partial x_{1}}\left(x_{1}, x_{2}\right), \frac{1}{\epsilon} \frac{\partial \varphi}{\partial x_{2}}\left(x_{1}, x_{2}\right)\right) d x_{1} d x_{2}=\int_{\Omega^{\epsilon}} \frac{\partial \varphi}{\partial x_{1}}\left(x_{1}, x_{2}\right) d x_{1} d x_{2} \\
=\int_{\Omega^{\epsilon}} \operatorname{div}(\varphi, 0) d x_{1} d x_{2}=\int_{\partial \Omega^{\epsilon}} \varphi N_{1}^{\epsilon} d S \\
=-\int_{0}^{1} \varphi\left(x, g\left(\frac{x}{\epsilon}\right)\right)\left(\frac{-\epsilon^{-1} g^{\prime}\left(\frac{x}{\epsilon}\right)}{\sqrt{1+\left(\epsilon^{-1} g\left(\frac{x}{\epsilon}\right)\right)^{2}}}\right) \sqrt{1+\left(\epsilon^{-1} g\left(\frac{x}{\epsilon}\right)\right)^{2}} d x \\
=\int_{0}^{1} \varphi\left(x, \frac{x}{\epsilon}\right) \frac{1}{\epsilon} g^{\prime}\left(\frac{x}{\epsilon}\right) d x=\int_{0}^{\epsilon k_{0} L} \varphi\left(x, \frac{x}{\epsilon}\right) \frac{1}{\epsilon} g^{\prime}\left(\frac{x}{\epsilon}\right) d x .
\end{gathered}
$$


De fato fazendo a mudança de variáveis $\left(x_{1}, x_{2}\right)=\psi_{k}^{\epsilon}\left(y_{1}, y_{2}\right)=\left(\epsilon\left(y_{1}+k L\right), y_{2}\right)$ obtemos

$$
\begin{aligned}
& \int_{\Omega^{\epsilon} \cap A_{k}^{\epsilon}} \nabla X\left(T_{k}^{\epsilon}\left(x_{1}, x_{2}\right)\right)\left(\frac{\partial \varphi}{\partial x_{1}}\left(x_{1}, x_{2}\right), \frac{1}{\epsilon} \frac{\partial \varphi}{\partial x_{2}}\left(x_{1}, x_{2}\right)\right) d x_{1} d x_{2} \\
= & \int_{Y^{*}} \nabla X\left(y_{1}, y_{2}\right)\left(\epsilon \frac{\partial \varphi}{\partial x_{1}}\left(\psi_{k}^{\epsilon}\left(y_{1}, y_{2}\right)\right), \frac{\partial \varphi}{\partial x_{2}}\left(\psi_{k}^{\epsilon}\left(y_{1}, y_{2}\right)\right)\right) d y_{1} d y_{2} \\
= & \int_{Y^{*}} \nabla X\left(y_{1}, y_{2}\right) \nabla\left(\varphi \circ \psi_{k}^{\epsilon}\right)\left(y_{1}, y_{2}\right) d y_{1} d y_{2}=\int_{\partial Y^{*}} \varphi \circ \psi_{k}^{\epsilon} \frac{\partial X}{\partial N} d S \\
= & -\int_{0}^{L} \varphi\left(\psi_{k}^{\epsilon}(y, g(y))\right)\left(\frac{-g^{\prime}(y)}{\sqrt{1+\left(g^{\prime}(y)\right)^{2}}}\right)\left(\sqrt{1+\left(g^{\prime}(y)\right)^{2}} d y\right) \\
= & \int_{0}^{L} \varphi(\epsilon(y+k L), g(y)) g^{\prime}(y) d y=\int_{\epsilon k L}^{\epsilon(k+1) L} \varphi\left(x, g\left(\frac{x}{\epsilon}\right)\right) g^{\prime}\left(\frac{x}{\epsilon}\right) \frac{d x}{\epsilon} .
\end{aligned}
$$

Comparando (3.31) e (3.32) obtemos o resultado desejado.

Agora estamos prontos para alterar a equação (3.21) de modo que possamos carcular o limite. Seja $\phi\left(x_{1}\right) \in C_{0}^{\infty}(0,1)$ e considere as funções-testes $\varphi=\phi \omega^{\epsilon}$ (funções-teste oscilantes de Tartar) em (3.21) e em (3.30). Então

$$
\begin{aligned}
\int_{\Omega^{\epsilon}} f^{\epsilon}\left(\phi \omega^{\epsilon}\right) d x_{1} d x_{2}=\int_{\Omega^{\epsilon}} & \left\{\frac{\partial u^{\epsilon}}{\partial x_{1}} \frac{\partial \phi}{\partial x_{1}} \omega^{\epsilon}+\frac{\partial u^{\epsilon}}{\partial x_{1}} \frac{\partial \omega^{\epsilon}}{\partial x_{1}} \phi+\frac{1}{\epsilon^{2}} \frac{\partial u^{\epsilon}}{\partial x_{2}} \frac{\partial \omega^{\epsilon}}{\partial x_{2}} \phi\right. \\
& +V^{\epsilon} u^{\epsilon} \phi \omega^{\epsilon}-\eta_{1}^{\epsilon} \frac{\partial \phi}{\partial x_{1}} u^{\epsilon} \\
& \left.-\eta_{1}^{\epsilon} \frac{\partial u^{\epsilon}}{\partial x_{1}} \phi-\frac{1}{\epsilon^{2}} \eta_{2}^{\epsilon} \frac{\partial u^{\epsilon}}{\partial x_{2}} \phi\right\} d x_{1} d x_{2} .
\end{aligned}
$$

Usando que $\eta_{i}^{\epsilon}=\frac{\partial \omega^{\epsilon}}{\partial x_{i}}$, cancelamos alguns termos apropriados e obtemos

$$
\int_{\Omega^{\epsilon}}\left\{\frac{\partial u^{\epsilon}}{\partial x_{1}} \frac{\partial \phi}{\partial x_{1}} \omega^{\epsilon}-\eta_{1}^{\epsilon} \frac{\partial \phi}{\partial x_{1}} u^{\epsilon}+V^{\epsilon} u^{\epsilon} \phi \omega^{\epsilon}\right\} d x_{1} d x_{2}=\int_{\Omega^{\epsilon}} f^{\epsilon} \phi \omega^{\epsilon} d x_{1} d x_{2},
$$

para toda $\phi$ em $C_{0}^{\infty}(0,1)$.

Por outro lado, temos obtido a formulação fraca (3.21) que é

$$
\int_{\Omega^{\epsilon}}\left\{\frac{\partial u^{\epsilon}}{\partial x_{1}} \frac{\partial \varphi}{\partial x_{1}}+\frac{1}{\epsilon^{2}} \frac{\partial \varphi}{\partial x_{2}} \frac{\partial u^{\epsilon}}{\partial x_{2}}+V^{\epsilon} u^{\epsilon} \varphi\right\} d x_{1} d x_{2}=\int_{\Omega^{\epsilon}} f^{\epsilon} \varphi d x_{1} d x_{2}, \forall \varphi \in H^{1}\left(\Omega^{\epsilon}\right)
$$

Agora precisamos passar o limite em (3.34) e (3.35). Para isto escreveremos ambas as expressões integrais sobre o mesmo domínio. Usaremos para isto a extensão $P_{\epsilon}$ construída no Lema (2.3.1), a extensão por zero (que denotaremos por $\sim$ ) e a função característica $\chi^{\epsilon}$ 
de $\Omega^{\epsilon}$ como segue:

$$
\begin{aligned}
& \int_{\Omega}\left\{\widetilde{\frac{\partial u^{\epsilon}}{\partial x_{1}}} \frac{\partial \phi}{\partial x_{1}} \omega^{\epsilon}-\widetilde{\eta_{1}^{\epsilon}} \frac{\partial \phi}{\partial x_{1}} P_{\epsilon} u^{\epsilon}+\chi^{\epsilon} P_{\epsilon}\left(V^{\epsilon}\right) P_{\epsilon}\left(u^{\epsilon}\right) \phi \omega^{\epsilon}\right\} d x_{1} d x_{2} \\
& =\int_{\Omega} \chi^{\epsilon} f^{\epsilon} \phi \omega^{\epsilon} d x_{1} d x_{2}, \quad \forall \phi \in C_{0}^{\infty}(0,1) \\
& \int_{\Omega}\left\{\widetilde{\frac{\partial u^{\epsilon}}{\partial x_{1}}} \frac{\partial \varphi}{\partial x_{1}}+\chi^{\epsilon} P_{\epsilon}\left(V^{\epsilon}\right) P_{\epsilon}\left(u^{\epsilon}\right) \varphi\right\} d x_{1} d x_{2}=\int_{\Omega} \chi^{\epsilon} f^{\epsilon} \varphi d x_{1} d x_{2}, \quad \forall \varphi \in H^{1}(0,1) .
\end{aligned}
$$

Para passarmos o limite nas equações acima, calcularemos o limite de algumas funções que formam o integrando.

\subsubsection{Limite das funções periódicas auxiliares}

(I). Limite de $\chi^{\epsilon}$

De (3.23) e do Teorema 2.2.1 obtemos

$$
\chi^{\epsilon}\left(\cdot, x_{2}\right) \stackrel{*}{\rightarrow} \theta:=\frac{1}{L} \int_{0}^{L} \chi\left(s, x_{2}\right) d s, \quad \text { em } L^{\infty}(I), \forall x_{2} \in\left(0, g_{1}\right) .
$$

Note que o limite $\theta$ não depende da variável $x_{2}$ e podemos obter a área do conjunto $Y^{*} \operatorname{com}$ a fórmula

$$
L \int_{0}^{g_{1}} \theta\left(x_{2}\right) d x_{2}=\left|Y^{*}\right|
$$

Se $\varphi \in L^{1}(\Omega)$, então segue do Teorema de Fubini-Tonelly que $\varphi\left(\cdot, x_{2}\right) \in L^{1}(0,1)$ para quase todo $x_{2} \in\left(0, g_{1}\right)$. Assim, de (3.37) temos que

$$
H^{\epsilon}\left(x_{2}\right)=\int_{I} \varphi\left(x_{1}, x_{2}\right)\left\{\chi^{\epsilon}\left(x_{1}, x_{2}\right)-\theta\left(x_{2}\right)\right\} d x_{1} \rightarrow 0 \text { quando } \epsilon \rightarrow 0
$$

para quase todo $x_{2} \in\left(0, g_{1}\right)$. Como $\left|\chi^{\epsilon}-\theta\right| \leq 1$, segue que

$$
\left|H^{\epsilon}\left(x_{2}\right)\right| \leq \int_{I}\left|\varphi\left(x_{1}, x_{2}\right)\right| d x_{1} \text { q.s em }\left(0, g_{1}\right)
$$

e portanto segue do Teorema da Convergência Dominada de Lebesgue que

$$
\chi^{\epsilon} \stackrel{*}{\rightarrow} \theta, \quad \text { em } L^{\infty}(\Omega)
$$

\section{(II). Limite das funções estendidas por zero}

Como $\left\|f^{\epsilon}\right\|_{L^{2}(\Omega)}$ é uniformemente limitada, obtemos de (3.22) que existe $M$ independente de 
$\epsilon$ tal que

$$
\left\|\widetilde{u^{\epsilon}}\right\|_{L^{2}(\Omega)},\left\|\widetilde{\frac{\partial u^{\epsilon}}{\partial x_{1}}}\right\|_{L^{2}(\Omega)}, \frac{1}{\epsilon}\left\|\widetilde{\frac{\partial u^{\epsilon}}{\partial x_{2}}}\right\|_{L^{2}(\Omega)} \leq M, \forall \epsilon>0 .
$$

Então existe uma subsequência, que denotaremos por $\widetilde{u^{\epsilon}}, \frac{\widetilde{\partial u^{\epsilon}}}{\partial x_{1}}$ e $\frac{\widetilde{\partial u^{\epsilon}}}{\partial x_{2}}$ tais que

$$
\begin{gathered}
\frac{\tilde{u}^{\epsilon}}{\frac{\partial u^{\epsilon}}{\partial x_{1}}} \rightarrow u^{*} \text { em } L^{2}(\Omega) \\
\frac{\partial u^{\epsilon}}{\partial x_{2}} \rightarrow 0 \text { em } L^{2}(\Omega) \text { e } \\
L^{2}(\Omega)
\end{gathered}
$$

quando $\epsilon \rightarrow 0$ para algum $u^{*}$ e $\xi^{*} \in L^{2}(\Omega)$.

Mais ainda, como $\left\|f^{\epsilon}\right\|_{L^{2}\left(\Omega^{\epsilon}\right)} \leq C$ independente de $\epsilon$, existe uma subsequência (que denotaremos por $\epsilon$ )tal que $\tilde{f}^{\epsilon} \rightarrow \widetilde{f}$ para alguma $\tilde{f} \in L^{2}(\Omega)$. Então para cada $\varphi \in L^{2}(I)$ temos

$$
\int_{\Omega} \tilde{f}^{\epsilon}\left(x_{1}, x_{2}\right) \varphi\left(x_{1}\right) d x_{1} d x_{2} \rightarrow \int_{\Omega} \tilde{f}\left(x_{1}, x_{2}\right) \varphi\left(x_{1}\right) d x_{1} d x_{2}
$$

Logo

$$
\widehat{f}^{\epsilon}\left(x_{1}\right):=\int_{0}^{g_{1}} \tilde{f}^{\epsilon}\left(x_{1}, x_{2}\right) d x_{2}=\int_{0}^{g\left(x_{1} / \epsilon\right)} f^{\epsilon}\left(x_{1}, x_{2}\right) d x_{2}
$$

satisfaz $\widehat{f}^{\epsilon} \in L^{2}(0,1)$ (segue do Teorema de Fubini-Tonelly) e portanto,

$$
\widehat{f} \epsilon \rightarrow \widehat{f} \mathrm{em} L^{2}(0,1)
$$

onde

$$
\widehat{f}\left(x_{1}\right):=\int_{0}^{g_{1}} \tilde{f}\left(x_{1}, x_{2}\right) d x_{2}=\int_{0}^{g\left(x_{1} / \epsilon\right)} \tilde{f}\left(x_{1}, x_{2}\right) d x_{2}
$$

Observação. Note que se $f^{\epsilon}\left(x_{1}, x_{2}\right)=f\left(x_{1}\right)$, então

$$
\widehat{f}\left(x_{1}\right)=\left(\frac{1}{L} \int_{0}^{L} g(x) d x\right) f\left(x_{1}\right)=\frac{\left|Y^{*}\right|}{L} f\left(x_{1}\right)
$$

pois neste caso $\widehat{f} \epsilon\left(x_{1}\right)=g\left(x_{1} / \epsilon\right) f\left(x_{1}\right)$.

\section{(III). Limite das funções extendidas}

Usando (3.22), fato de que $u^{\epsilon} \in H^{1}\left(\Omega^{\epsilon}\right)$ e usando os resultados do Lema 3.3.1 sobre o operador extensão, obtemos

$$
\left\|P_{\epsilon} u^{\epsilon}\right\|_{L^{2}(\Omega)},\left\|\frac{\partial P_{\epsilon} u^{\epsilon}}{\partial x_{1}}\right\|_{L^{2}(\Omega)}, \frac{1}{\epsilon}\left\|\frac{\partial P_{\epsilon} u^{\epsilon}}{\partial x_{2}}\right\|_{L^{2}(\Omega)} \leq \widetilde{M}, \forall \epsilon>0 .
$$


onde $\widetilde{M}>0$ é uma constante independente de $\epsilon$ e dada pela estimativa (3.41) e pelo Lema 3.3.1. Então podemos tomar uma subsequência, que será denotada por $P_{\epsilon} u^{\epsilon}$ e uma função $u_{0} \in H^{1}(\Omega)$, tal que

$$
\begin{gathered}
P_{\epsilon} u^{\epsilon} \rightarrow u_{0} \text { em } H^{1}(\Omega) \text { e } P_{\epsilon} u^{\epsilon} \rightarrow u_{0} \text { em } L^{p}(\Omega) \forall p \geq 1 \\
\frac{\partial P_{\epsilon} u^{\epsilon}}{\partial x_{2}} \rightarrow 0 \text { em } L^{2}(\Omega) .
\end{gathered}
$$

aqui, a convergencia $P_{\epsilon} u^{\epsilon} \rightarrow u_{0}$ em $L^{p}(\Omega) \forall p \geq 1$ segue das Imersões de Sobolev (Teorema 2.3.1).

Uma consequência dos limites (3.46) é que $u_{0}\left(x_{1}, x_{2}\right)=u_{0}\left(x_{1}\right)$ em $\Omega$. Mais precisamente,

$$
\frac{\partial u_{0}}{\partial x_{2}}\left(x_{1}, x_{2}\right)=0 \text { q. s. em } \Omega
$$

De fato, para todo $\varphi \in C_{0}^{\infty}(\Omega)$, temos por (3.46) que

$$
\int_{\Omega} u_{0} \frac{\partial \varphi}{\partial x_{2}} d x_{1} d x_{2}=\lim _{\epsilon \rightarrow 0} \int_{\Omega} P_{\epsilon} u^{\epsilon} \frac{\partial \varphi}{\partial x_{2}} d x_{1} d x_{2}=-\lim _{\epsilon \rightarrow 0} \int_{\Omega} \frac{\partial P_{\epsilon} u^{\epsilon}}{\partial x_{2}} \varphi d x_{1} d x_{2}=0 .
$$

Em particular, podemos considerar que $u_{0} \in H^{1}(0,1)$.

Também note que $\widetilde{u^{\epsilon}}=\chi^{\epsilon} P_{\epsilon} u^{\epsilon}$ q. s. em $\Omega$. Como $\chi^{\epsilon} \stackrel{*}{\rightarrow} \theta$ em $\left(L^{1}(\Omega)\right)^{*}$ e $\chi^{\epsilon}, \theta \in L^{2}(\Omega)$ (pois $\Omega$ é limitado), segue que $\chi^{\epsilon} \rightarrow \theta$ em $L^{2}(\Omega)$. Mas $\widetilde{u^{\epsilon}} \rightarrow u^{*}$ em $L^{2}(\Omega)$ e $P_{\epsilon} u^{\epsilon} \rightarrow u_{0}$ em $L^{2}(\Omega)$. Logo, $\chi^{\epsilon} P_{\epsilon} u^{\epsilon} \rightarrow \theta u_{0}$ em $L^{2}(\Omega)$ e portanto

$$
u^{*}\left(x_{1}, x_{2}\right)=\theta\left(x_{2}\right) u_{0}\left(x_{1}\right) \text { q. s. em } \Omega \text {. }
$$

\section{(IV). Limite de $\omega^{\epsilon}$}

Com a definição de $\omega^{\epsilon}$, sabendo que $\left|\operatorname{det}\left(T_{k}^{\epsilon}\right)^{\prime}\left(x_{1}, x_{2}\right)\right|=\epsilon^{-1}$, segue do Teorema de Mudança de Variáveis e do Lema 3.3.1 que

$$
\int_{A_{k}^{\epsilon}}\left|\omega^{\epsilon}-x_{1}\right|^{2} d x_{1} d x_{2}=\int_{Y} \epsilon^{3}\left|(P X)\left(y_{1}, y_{2}\right)\right|^{2} d x y_{1} d y_{2} \leq \int_{Y^{*}} C \epsilon^{3}\left|X\left(y_{1}, y_{2}\right)\right|^{2} d y_{1} d y_{2}
$$

e também,

quando $\epsilon \rightarrow 0$. Similarmente,

$$
\begin{aligned}
\int_{\Omega}\left|\omega^{\epsilon}-x_{1}\right|^{2} d x_{1} d x_{2} & \cong \sum_{k=1}^{\frac{1}{\epsilon L}} C \epsilon^{3} \int_{Y^{*}}\left|X\left(y_{1}, y_{2}\right)\right|^{2} d y_{1} d y_{2} \\
& \cong \epsilon^{2} \int_{Y^{*}} C\left|X\left(y_{1}, y_{2}\right)\right|^{2} d y_{1} d y_{2} \rightarrow 0
\end{aligned}
$$

$$
\begin{aligned}
\int_{A_{k}^{\epsilon}}\left|\frac{\partial}{\partial x_{1}}\left(\omega^{\epsilon}-x_{1}\right)\right|^{2} d x_{1} d x_{2} & =\int_{Y}\left|\frac{\partial P X}{\partial y_{1}}\left(y_{1}, y_{2}\right)\right|^{2} \epsilon d y_{1} d y_{2} \\
& \leq \epsilon \int_{Y^{*}} C\left|\frac{\partial X}{\partial y_{1}}\left(y_{1}, y_{2}\right)\right|^{2} d y_{1} d y_{2}
\end{aligned}
$$


e

$$
\begin{aligned}
\int_{A_{k}^{\epsilon}}\left|\frac{\partial}{\partial x_{2}}\left(\omega^{\epsilon}-x_{1}\right)\right|^{2} d x_{1} d x_{2} & =\int_{Y}\left|\frac{\partial P X}{\partial y_{2}}\left(y_{1}, y_{2}\right) \epsilon\right|^{2} d y_{1} d y_{2} \\
& \leq \epsilon^{3} \int_{Y^{*}} C\left|\frac{\partial X}{\partial y_{2}}\left(y_{1}, y_{2}\right)\right|^{2} d y_{1} d y_{2}
\end{aligned}
$$

Assim, temos

$$
\begin{aligned}
\int_{\Omega}\left|\frac{\partial}{\partial x_{1}}\left(\omega^{\epsilon}-x_{1}\right)\right|^{2} d x_{1} d x_{2} & \cong \sum_{k=1}^{\frac{1}{\epsilon L}} C \epsilon \int_{Y^{*}}\left|\frac{\partial X}{\partial y_{1}}\left(y_{1}, y_{2}\right)\right|^{2} d y_{1} d y_{2} \\
& \cong \int_{Y^{*}} \widetilde{C}\left|\frac{\partial X}{\partial y_{1}}\left(y_{1}, y_{2}\right)\right|^{2} d y_{1} d y_{2}
\end{aligned}
$$

para todo $\epsilon>0$ e

$$
\int_{\Omega}\left|\frac{\partial}{\partial x_{2}}\left(\omega^{\epsilon}-x_{1}\right)\right|^{2} d x_{1} d x_{2} \leq \epsilon^{2} \int_{Y^{*}} \widetilde{C}\left|\frac{\partial X}{\partial y_{2}}\left(y_{1}, y_{2}\right)\right|^{2} d y_{1} d y_{2} \rightarrow 0 \text { quando } \epsilon \rightarrow 0
$$

Assim, usando as Imesões de Sobolev, concluímos

$$
\begin{gathered}
\omega^{\epsilon} \rightarrow x_{1} \text { em } H^{1}(\Omega) \text { e } \omega^{\epsilon} \rightarrow x_{1} \text { em } L^{p}(\Omega), \forall p \geq 1 \\
\frac{\partial \omega^{\epsilon}}{\partial x_{2}} \rightarrow 0 \text { em } L^{2}(\Omega) .
\end{gathered}
$$

(V). Limite de $\eta_{i}^{\epsilon}$

Seja $\widetilde{\eta^{\epsilon}}$ a extensão por zero do vetor $\eta^{\epsilon}$ para todo $\Omega$. Claramente, $\widetilde{\eta}^{\epsilon}$ é periodica na primeira variável. Assim, da definição de $\eta_{1}^{\epsilon}$ em (3.29) segue que

$$
\left.\widetilde{\eta_{1}^{\epsilon}}\left(x_{1}, x_{2}\right)=\chi\left(\frac{x_{1}}{\epsilon}, x_{2}\right)-\widetilde{\partial X} \frac{x_{1}}{\partial y_{1}}, x_{2}\right)
$$

e portanto, sabendo que $\frac{\partial X}{\partial y_{1}} \in L^{\infty}\left(Y^{*}\right)$ (isto segue do Lema B.1, vi) de [20]), segue do Teorema 2.2.1 que

$$
\widetilde{\eta_{1}^{\epsilon}}\left(x_{1}, x_{2}\right) \stackrel{*}{\rightarrow} \frac{1}{L} \int_{0}^{L}\left(1-\frac{\widetilde{\partial X}}{\partial y_{1}}\left(s, x_{2}\right)\right) \chi\left(s, x_{2}\right) d s:=q\left(x_{2}\right), \text { em } L^{\infty}(I) .
$$

onde $\chi$ é a função característica de $Y^{*}$. Assim, argumentando como em (3.40) e notando que podemos assumir que $\left|\frac{\partial X}{\partial y_{1}}\left(y_{1}, y_{2}\right)\right| \leq\left\|\frac{\partial X}{\partial y_{1}}\right\|_{L^{\infty}\left(Y^{*}\right)}$ para todo $\left(y_{1}, y_{2}\right) \in Y^{*}$, podemos provar que

$$
\widetilde{\eta_{1}^{\epsilon}} \stackrel{*}{\rightarrow} q \text { em } L^{\infty}(\Omega)
$$




\subsubsection{Convergência para o limite homogeneizado}

Agora, pelas convergências dadas em $(I)-(V)$ acima, podemos passar o limite. Note primeiro que $\chi^{\epsilon} \stackrel{*}{\rightarrow} \theta$ em $L^{\infty}(\Omega), P_{\epsilon} u^{\epsilon} \rightarrow u_{0}$ e $\omega^{\epsilon} \rightarrow x_{1}$ em $L^{2}(\Omega)$, e $P_{\epsilon} V^{\epsilon} \rightarrow V_{0}$ em $L^{\infty}(\Omega)$ (segue de (3.20)). Assim, usando a desigualdade de Hölder, obtemos

$$
P_{\epsilon} V^{\epsilon} P_{\epsilon} u^{\epsilon} \phi \omega^{\epsilon} \rightarrow V_{0} u_{0} \phi x_{1} \text { em } L^{1}(\Omega)
$$

e portanto

$$
\int_{\Omega^{\epsilon}} V^{\epsilon} u^{\epsilon} \phi \omega^{\epsilon}=\int_{\Omega} \chi^{\epsilon} P_{\epsilon} V^{\epsilon} P_{\epsilon} u^{\epsilon} \phi \omega^{\epsilon} \rightarrow \int_{\Omega} \theta V_{0} u_{0} \phi x_{1}
$$

Assim, passando o limite nas equações (3.36), obtemos

$$
\begin{array}{r}
\int_{\Omega}\left\{\xi^{*}\right. \\
\left.\frac{\partial \phi}{\partial x_{1}} x_{1}-q \frac{\partial \phi}{\partial x_{1}} u_{0}+\theta V_{0} u_{0} \phi x_{1}\right\} d x_{1} d x_{2} \\
=\int_{0}^{1} \widehat{f}\left(x_{1}\right) \phi\left(x_{1}\right) x_{1} d x_{1}, \quad \forall \phi \in C_{0}^{\infty}(0,1)
\end{array}
$$

Note que $\xi^{*} \frac{\partial}{\partial x_{1}}\left(\phi x_{1}\right)=\xi^{*} x_{1} \frac{\partial \phi}{\partial x_{1}}+\xi^{*} \phi$. Consequentemente,

$$
\int_{\Omega}\left\{\xi^{*} \frac{\partial}{\partial x_{1}}\left(\phi x_{1}\right)-\phi \xi^{*}-q \frac{\partial \phi}{\partial x_{1}} u_{0}+\theta V_{0} u_{0} \phi x_{1}\right\} d x_{1} d x_{2}=\int_{0}^{1} \widehat{f}\left(x_{1}\right) \phi\left(x_{1}\right) x_{1} d x_{1}
$$

para todo $\phi \in C_{0}^{\infty}(0,1)$. De (3.36) obtemos

$$
\int_{\Omega}\left\{\xi^{*} \frac{\partial \varphi}{\partial x_{1}}+\theta V_{0} u_{0} \varphi\right\} d x_{1} d x_{2}=\int_{0}^{1} \widehat{f} \varphi d x_{1}, \forall \varphi \in H^{1}(0,1) .
$$

Em particular, usando o Teorema de Fubini e (3.38) obtemos para cada $\varphi \in H^{1}(0,1)$

$$
\int_{0}^{1}\left\{\left(\int_{0}^{g_{1}} \xi^{*}\left(x_{1}, x_{2}\right) d x_{2}\right) \frac{\partial \varphi}{\partial x_{1}}+\frac{\left|Y^{*}\right|}{L} V_{0} u_{0}\left(x_{1}\right) \varphi\left(x_{1}\right)\right\} d x_{1}=\int_{0}^{1} \widehat{f}\left(x_{1}\right) \varphi\left(x_{1}\right) d x_{1}
$$

Tomando $\varphi=\phi x_{1}$ em (3.53), obtemos

$$
\int_{\Omega}\left\{\xi^{*} \frac{\partial}{\partial x_{1}}\left(\phi x_{1}\right)+\theta V_{0} u_{0} \phi x_{1}\right\} d x_{1} d x_{2}=\int_{0}^{1} \widehat{f} \phi x_{1} d x_{1}
$$

Assim, segue de (3.52) e (3.55) que, para toda $\phi \in C_{0}^{\infty}(0,1)$,

$$
0=\int_{\Omega}\left\{\phi \xi^{*}+q \frac{\partial \phi}{\partial x_{1}} u_{0}\right\} d x_{1} d x_{2}=\int_{\Omega}\left\{\phi \xi^{*}-q \phi \frac{\partial u_{0}}{\partial x_{1}}\right\} d x_{1} d x_{2}
$$

onde obtemos a última integral por integração por partes. Assim, se definirmos

$$
\widehat{q}:=\int_{0}^{g_{1}} q(s) d s=\frac{1}{L} \int_{Y^{*}}\left\{1-\frac{\partial X}{\partial y_{1}}\right\} d y_{1} d y_{2}
$$


e aplicando integrais iteradas em (3.56) obtemos

$$
\int_{0}^{1} \phi\left(x_{1}\right)\left(\int_{0}^{g_{1}} \xi^{*}\left(x_{1}, x_{2}\right) d x_{2}-\widehat{q} \frac{\partial u_{0}}{\partial x_{1}} d x_{1}=0, \quad \forall \phi \in C_{0}^{\infty}(0,1)\right)
$$

o que implica que

$$
\int_{0}^{g_{1}} \xi^{*}\left(x_{1}, x_{2}\right) d x_{2}=\widehat{q} \frac{\partial u_{0}}{\partial x_{1}}, \text { q. s. } x \in(0,1)
$$

Usando esta última equação em (3.54) obtemos

$$
\int_{0}^{1}\left\{\widehat{q} \frac{\partial u_{0}}{\partial x_{1}} \frac{\partial \varphi}{\partial x_{1}}+\frac{\left|Y^{*}\right|}{L} V_{0} u_{0} \varphi\right\} d x_{1}=\int_{0}^{1} \widehat{f} \varphi d x_{1}, \quad \forall \varphi \in H^{1}(0,1)
$$

Assim, se definirmos $q_{0}=\frac{L}{\left|Y^{*}\right|} \widehat{q}$, que é

$$
q_{0}=\frac{1}{\left|Y^{*}\right|} \int_{Y^{*}}\left\{1-\frac{\partial X}{\partial y_{1}}\left(y_{1}, y_{2}\right)\right\} d y_{1} d y_{2}
$$

e também

obtemos de (3.59)

$$
f_{0}\left(x_{1}\right)=\frac{L}{\left|Y^{*}\right|} \widehat{f}\left(x_{1}\right)
$$

$$
\int_{0}^{1}\left(q_{0} \frac{\partial u_{0}}{\partial x_{1}} \frac{\partial \varphi}{\partial x_{1}}+V_{0} u_{0} \varphi\right) d x_{1}=\int_{0}^{1} f_{0} \varphi d x_{1}, \forall \varphi \in H^{1}(0,1)
$$

que é a formulação fraca de

$$
\left\{\begin{array}{c}
-q_{0} u_{0 x x}+V_{0} u_{0}=f_{0} \text { em }(0,1) \\
u_{0}^{\prime}(0)=u_{0}^{\prime}(1)=0
\end{array}\right.
$$

Observação 3.4.1. Note que em relação a observação anterior, se originalmente o termo não homogêneo $f$ não depende de $x_{2}$, que é, $f\left(x_{1}, x_{2}\right)=f\left(x_{1}\right)$, então com a definição de $f_{0}$, e com (3.38) temos

$$
f_{0}\left(x_{1}\right)=\frac{L}{\left|Y^{*}\right|} \int_{0}^{g_{1}} \theta(s) f\left(x_{1}\right) d s=\frac{L}{\left|Y^{*}\right|} \frac{\left|Y^{*}\right|}{L} f\left(x_{1}\right)=f\left(x_{1}\right) .
$$

Assim, a equação (3.63) com $V_{0} \equiv 1$ (que é $V^{\epsilon} \equiv 1$ ), está de acordo com o enocntrado através do método da múltipla escala.

Para concluimos esta seção, mostraremos que o problema (3.63) está bem posto no sentido de que o coeficiente $q_{0}>0$. Para isto, usamos a formulação variacional do problema auxiliar (3.26), que é, a forma bilinear

$$
b: H^{1}\left(Y^{*}\right) \times H^{1}\left(Y^{*}\right) \mapsto \mathbb{R}:(\phi, \varphi) \rightarrow \int_{Y^{*}} \nabla \phi \cdot \nabla \varphi d y_{1} d y_{2} .
$$


Assim, para toda $\phi \in H^{1}\left(Y^{*}\right)$ que é L-periódica na variável $y_{1}$, obtemos que $X$ satisfaz

$$
b(X, \phi)=-\int_{B_{1}} \frac{g^{\prime}}{\sqrt{1+g^{\prime 2}}} \phi d S .
$$

onde $B_{1}$ é a parte superior da fronteira da célula básica. De fato, tomando $\vec{F}: Y^{*} \rightarrow \mathbb{R}^{2}$ dada por $\vec{F}=\left(\frac{\partial X}{\partial y_{1}} \phi, \frac{\partial X}{\partial y_{2}} \phi\right)$ e usando que $\Delta X=0$ em $Y^{*}$ obtemos

$$
\int_{Y^{*}} \nabla X \cdot \nabla \phi d y_{1} d y_{2}=\int_{Y^{*}} \operatorname{div}(\vec{F}) d y_{1} d y_{2}=\int_{\partial Y^{*}} \vec{F} \cdot \vec{\eta} d S
$$

Para concluir, basta notar que $\vec{F} \cdot \vec{\eta}=\frac{\partial X}{\partial N} \phi$ e usar sua periodicidade.

Analogamente, tomando $\vec{F}=(\phi,-1)$ obtemos

$$
b\left(y_{1}, \phi\right)=\int_{B_{1}} \phi N_{1} d S
$$

Consequentemente, $y_{1}-X$ satisfaz

$$
b\left(y_{1}-X, \phi\right)=\int_{B_{1}} N_{1} \phi d S+\int_{B_{1}} \frac{g^{\prime}}{\sqrt{1+g^{\prime 2}}} d S=0
$$

para toda $\phi \in H^{1}\left(Y^{*}\right)$ que é L-periódica na variável $y_{1}$, onde o vetor normal $N$ sobre $B_{1}$ é dado por

$$
N=\left(N_{1}, N_{2}\right)=\frac{\left(-g^{\prime}, 1\right)}{\sqrt{1+g^{\prime 2}}}
$$

Assim, obtemos por (3.60)

$$
q_{0}\left|Y^{*}\right|=\int_{Y^{*}} \frac{\partial}{\partial y_{1}}\left(y_{1}-X\right) \frac{\partial y_{1}}{\partial y_{1}} d y_{1} d y_{2}=b\left(y_{1}-X, y_{1}\right)
$$

Assim, de (3.64) com $\phi=-X$, da identidade (3.65) e da periodicidade de $X$, obtemos

$$
q_{0}\left|Y^{*}\right|=b\left(y_{1}-X, y_{1}\right)+b\left(y_{1}-X,-X\right)=\left\|\nabla\left(y_{1}-X\right)\right\|_{L^{2}\left(Y^{*}\right)}^{2}>0 .
$$

pois $\left\|\nabla\left(y_{1}-X\right)\right\|_{L^{2}\left(Y^{*}\right)}^{2}=0$ implica que existe $C \in \mathbb{R}$ constante tal que $X+C=y_{1}$, que é uma contradição pois $X+C$ é periódica e $f \equiv y_{1}$ não é periódica. Portanto segue que $q_{0}>0$ e o problema acima está bem posto. Em particular, obtemos unicidade de sulução do problema (3.63)

Resumimos esta seção no seguinte teorema com os resultados desta seção. 
Teorema 3.4.1. Seja $u^{\epsilon}$ solução de (3.19) com $V^{\epsilon} \in L^{\infty}\left(\Omega^{\epsilon}\right), V^{\epsilon} \geq 1$, e f $f^{\epsilon} \in L^{2}\left(\Omega^{\epsilon}\right)$, $\left\|f^{\epsilon}\right\|_{L^{2}\left(\Omega^{\epsilon}\right)} \leq C$ com $C$ independente de $\epsilon$. Então

(i) Se temos uma sequência $\epsilon \rightarrow 0$ tal que

$$
\widehat{f}^{\epsilon}(\cdot)=\int_{0}^{g\left(x_{1} / \epsilon\right)} f^{\epsilon}\left(\cdot, x_{2}\right) d x_{2} \rightarrow \widehat{f}(\cdot), \quad \text { em } L^{2}(0,1)
$$

$e$

$$
\left\|V^{\epsilon}-V_{0}\right\|_{L^{\infty}\left(\Omega^{\epsilon}\right)} \rightarrow 0 \text { quando } \epsilon \rightarrow 0
$$

onde $V_{0} \in L^{\infty}(\Omega)$ não depende da variável $x_{2}$. Então

$$
P_{\epsilon} u^{\epsilon} \rightarrow u_{0} \quad e m H^{1}(\Omega)
$$

onde $P_{\epsilon} \in \mathcal{L}\left(L^{2}\left(\Omega^{\epsilon}\right), L^{2}(\Omega)\right) \cap \mathcal{L}\left(H^{1}\left(\Omega^{\epsilon}\right), H^{1}(\Omega)\right)$ é o operador extensão definido no Lema $3.3 .1\left(\operatorname{com} \mathcal{O}_{\epsilon}=\Omega_{\epsilon}\right.$ e $\left.\mathcal{O}=\Omega\right)$ e $u_{0}\left(x_{1}, x_{2}\right)=u_{0}\left(x_{1}\right)$ para todo $\left(x_{1}, x_{2}\right) \in \Omega$ é a solução da formulação fraca de

$$
\left\{\begin{array}{c}
-q_{0} u_{0 x x}+V_{0} u_{0}=f_{0} \text { em }(0,1) \\
u_{0}^{\prime}(0)=u_{0}^{\prime}(1)=0
\end{array}\right.
$$

onde $q_{0}>0$ é dado por (3.60) e $f_{0}(\cdot)=\frac{L}{\left|Y^{*}\right|} \widehat{f}(\cdot)$.

(ii) Para qualquer sequência $\epsilon \rightarrow 0$, temos uma subsequência (que indexamos também por $\epsilon)$, tal que as funções $\widehat{f}^{\epsilon}(\cdot) \rightarrow \widehat{f}(\cdot)$ em $L^{2}(0,1)$ para alguma função $\widehat{f}$. Em particular, para esta subsequência temos o resultado de (i).

Observação 3.4.2. Em geral, a convergência $P_{\epsilon} u^{\epsilon} \rightarrow u_{0}$ em $H^{1}(\Omega)$ é a melhor que podemos obter. De fato, suponha que $P_{\epsilon} u^{\epsilon} \rightarrow u_{0}$ em $H^{1}(\Omega)$. Então

$$
\frac{\widetilde{\partial u^{\epsilon}}}{\partial x_{1}}=\frac{\partial\left(P_{\epsilon} u^{\epsilon}\right)}{\partial x_{1}} \chi^{\epsilon} \rightarrow \frac{d u_{0}}{d x_{1}} \theta=\xi^{*} \text { em } L^{2}(\Omega)
$$

e portanto teríamos

$$
\int_{0}^{g_{1}} \xi^{*}\left(x_{1}, x_{2}\right) d x_{1} d x_{2}=\int_{0}^{g_{1}} \frac{d u_{0}}{d x_{1}}\left(x_{1}\right) \theta\left(x_{2}\right) d x_{2}=\frac{d u_{0}}{d x_{1}}\left(x_{1}\right) \frac{\left|Y^{*}\right|}{L} \text { q. s. } x_{1} \in(0,1)
$$

assim, voltando em (3.58) obtemos

$$
\frac{d u_{0}}{d x_{1}}\left(x_{1}\right) \frac{\left|Y^{*}\right|}{L}=\widehat{q} \frac{d u_{0}}{d x_{1}}\left(x_{1}\right) \text { q. s. } x_{1} \in(0,1)
$$

e portanto

$$
\frac{\left|Y^{*}\right|}{L}=\widehat{q}=\frac{1}{L} \int_{Y^{*}}\left\{1-\frac{\partial X}{\partial y_{1}}\left(y_{1}, y_{2}\right)\right\} d y_{1} d y_{2}=\frac{\left|Y^{*}\right|}{L}-\frac{1}{L} \int_{Y^{*}} \frac{\partial X}{\partial y_{1}}\left(y_{1}, y_{2}\right) d y_{1} d y_{2},
$$


$\log 0$

$$
\int_{Y^{*}} \frac{\partial X}{\partial y_{1}}\left(y_{1}, y_{2}\right) d y_{1} d y_{2}=0
$$

Por outro lado,

$$
\begin{aligned}
& \int_{Y^{*}} \frac{\partial X}{\partial y_{1}}\left(y_{1}, y_{2}\right) d y_{1} d y_{2}=\int_{Y^{*}} \operatorname{div}(X, 0) d y_{1} d y_{2}=\int_{\partial Y^{*}}(X, 0) \cdot N d s=\int_{\partial Y^{*}} X N_{1} d s \\
& =-\int_{0}^{L} X(y, g(y))\left(\frac{-g^{\prime}(y)}{\sqrt{1+\left(g^{\prime}(y)\right)^{2}}}\right)\left(\sqrt{1+\left(g^{\prime}(y)\right)^{2}} d y\right)=\int_{0}^{L} X(y, g(y)) g^{\prime}(y) d y
\end{aligned}
$$

Assim, fazendo $\varphi=X$ em (3.27) obtemos

$$
\|\nabla X\|_{L^{2}\left(Y^{*}\right)}^{2}=\int_{Y^{*}} \nabla X \cdot \nabla X d y_{1} d y_{2}=\int_{0}^{L} X(y, g(y)) g^{\prime}(y) d y=\int_{Y^{*}} \frac{\partial X}{\partial y_{1}}\left(y_{1}, y_{2}\right) d y_{1} d y_{2}=0
$$

que por sua vez implica que $X=0$. Voltando em (3.27) concluimos que $g^{\prime}=0$ e portanto $g$ é constante. Logo, para os casos em que g não é constante, não é válida a convergência $P_{\epsilon} u^{\epsilon} \rightarrow u_{0}$ em $H^{1}(\Omega)$.

\subsection{Convergência do Resolvente}

Seja $\left\{Z_{\epsilon}\right\}_{\epsilon>0}$ uma família de espaços de Hilbert definidos por $Z_{\epsilon}=L^{2}\left(\Omega^{\epsilon}\right)$ com o produto interno canônico

$$
(u, v)_{\epsilon}=\int_{\Omega^{\epsilon}} u(x) v(x) d x
$$

e seja $Z_{0}=L^{2}(I)$ o espaço de Hilber limite com o produto interno dado por

$$
(u, v)_{Z_{0}}=\mu(g) \int_{0}^{1} u(x) v(x) d x
$$

onde

$$
\mu(g)=\frac{1}{L} \int_{0}^{L} g(s) d s
$$

Podemos escrever (3.19) como uma equação abstrata $L_{\epsilon} u=f^{\epsilon}$ onde $L_{\epsilon}: \mathcal{D}\left(L_{\epsilon}\right) \subset$ $L^{2}\left(\Omega^{\epsilon}\right) \rightarrow L^{2}\left(\Omega^{\epsilon}\right)$ é um operador linear, positivo, auto-adjunto, com resolvente compacto, definido por

$$
\begin{gathered}
\mathcal{D}\left(L_{\epsilon}\right)=\left\{u \in H^{1}\left(\Omega^{\epsilon}\right) ; \exists f \in L^{2}\left(\Omega^{\epsilon}\right) \text { satisfazendo } a_{\epsilon}(u, \varphi)=(f, \varphi)_{\epsilon}, \forall \varphi \in H^{1}\left(\Omega^{\epsilon}\right)\right\} \\
L_{\epsilon} u=f \in L^{2}\left(\Omega^{\epsilon}\right) \text { se } a_{\epsilon}(u, \varphi)=(f, \varphi)_{\epsilon}, \forall \varphi \in H^{1}\left(\Omega^{\epsilon}\right)
\end{gathered}
$$

onde $a_{\epsilon}: H^{1}\left(\Omega^{\epsilon}\right) \times H^{1}\left(\Omega^{\epsilon}\right) \rightarrow \mathbb{R}$ é uma forma bilinear, contínua, coerciva e simétrica definida por

$$
a_{\epsilon}(u, v)=\int_{\Omega^{\epsilon}}\left\{\frac{\partial u}{\partial x_{1}} \frac{\partial v}{\partial x_{1}}+\frac{1}{\epsilon^{2}} \frac{\partial u}{\partial x_{2}} \frac{\partial v}{\partial x_{2}}+V^{\epsilon} u v\right\} d x_{1} d x_{2} .
$$


De fato, segue do Teorema de Lax-Milgram que $L_{\epsilon}$ está bem definido, é bijetor e, como $L_{\epsilon}$ é simétrico, segue que é auto-adjunto. Como para todo $u \in \mathcal{D}\left(L_{\epsilon}\right),\left(-L_{\epsilon} u, u\right)_{\epsilon} \leq(-1)(u, u)_{\epsilon}$, segue que a imagem numérica de $L_{\epsilon}$ está contida em $(-\infty,-1]$ e portanto, do Corolário 1.1.1 segue que $L_{\epsilon}$ é positivo. A verificação de que $L_{\epsilon}$ tem resolvente compacto é uma consequência imediata do Teorema 3.4.1 e de que $H^{1}\left(\Omega^{\epsilon}\right)$ está compactamente imerso em $L^{2}\left(\Omega^{\epsilon}\right)$.

Analogamente, associamos o problema limite (3.63) ao operador linear limite $L_{0}: \mathcal{D}\left(L_{0}\right) \subset Z_{0} \rightarrow Z_{0}$ definido por

$$
\begin{gathered}
\mathcal{D}\left(L_{0}\right)=\left\{u \in H^{1}(0,1) ; \exists f \in L^{2}(0,1) \text { satisfazendo } a_{0}(u, \varphi)=(f, \varphi)_{0}, \forall \varphi \in H^{1}(0,1)\right\} \\
L_{0} u=f \in L^{2}(0,1) \text { se } a_{0}(u, \varphi)=(f, \varphi)_{0}, \forall \varphi \in H^{1}(0,1)
\end{gathered}
$$

onde $q_{0}>0$ é dado por $(3.60)$ e $a_{0}: H^{1}(0,1) \times H^{1}(0,1) \rightarrow \mathbb{R}$ é definido por

$$
a_{0}(u, v)=\int_{0}^{1}\left(q_{0} \frac{d u}{d x} \frac{d v}{d x}+V_{0} u v\right) d x, \forall u, v \in H^{1}(0,1),
$$

$\mathrm{e}$

$$
(u, v)_{0}:=\int_{0}^{1} u v d x, \forall u, v \in L^{2}(0,1)
$$

Note que $L_{0}$ é um operador linear, auto-adjunto, positivo e com resolvente compacto. Na seção anterior, nós passamos o limite na formulação variacional do problema (4.1) quando $\epsilon \rightarrow 0$ obtendo a equação limite (3.63). Aqui nós aplicamos o conceito de convergência compacta para obter propriedades de convergência de auto-valores e auto-funções quando o domínio oscilante se degenera para um segmento de reta.

Seja $E_{\epsilon}: Z_{0} \mapsto Z_{\epsilon}$ uma família de operadores lineares contínuos dados por

$$
\left(E_{\epsilon} u\right)\left(x_{1}, x_{2}\right)=u\left(x_{1}\right) \text { em } \Omega^{\epsilon}
$$

para $u \in Z_{0}$. Como

$$
\left\|E_{\epsilon} u\right\|_{Z_{\epsilon}}^{2}=\int_{0}^{1} g\left(x_{1} / \epsilon\right) u^{2}\left(x_{1}\right) d x_{1} \stackrel{\epsilon \rightarrow 0}{\rightarrow} \mu(g) \int_{0}^{1} u^{2}\left(x_{1}\right) d x_{1}=\|u\|_{Z_{0}}^{2},
$$

então temos $\left\|E_{\epsilon} u\right\|_{Z_{\epsilon}} \rightarrow\|u\|_{Z_{0}}$ quando $\epsilon \rightarrow 0$.

O seguinte corolário é um resultado importante para provarmos que o espectro de $L_{\epsilon}$ aproxima o espectro de $L_{0}$ quando $\epsilon \rightarrow 0$.

Corolário 3.5.1. A família de operadores compactos $\left\{L_{\epsilon}^{-1} \in \mathcal{L}\left(Z_{\epsilon}\right)\right\}_{\epsilon>0}$ converge compactamente para o operador compacto $L_{0}^{-1} \in \mathcal{L}\left(Z_{0}\right)$ quando $\epsilon \rightarrow 0$. 
Prova: Tome $\left\{f^{\epsilon}\right\}_{\epsilon>0}$ com $\left\|f^{\epsilon}\right\|_{Z_{\epsilon}} \leq 1$ e defina $u^{\epsilon}=L_{\epsilon}^{-1} f^{\epsilon}$. Assim, $L_{\epsilon} u^{\epsilon}=f^{\epsilon}$ e $u^{\epsilon}$ satisfaz o problema (3.19). Portanto segue do Teorema 3.4.1 que existe $f_{0} \in Z_{0}$ e $u_{0} \in H^{1}(\omega)$ tal que $P_{\epsilon} u^{\epsilon} \rightarrow u_{0}$ em $H^{1}(\omega)$ com $u_{0}\left(x_{1}, x_{2}\right)=u_{0}\left(x_{1}\right)$ q. s. em $\Omega$ satisfazendo $L_{0} u_{0}=f_{0}$, onde $P_{\epsilon}$ é o operador extensão dado pelo Lema 3.3.1. Assim, podemos concluir que

$$
\left\|u^{\epsilon}-E_{\epsilon} u_{0}\right\|_{Z_{\epsilon}}=\left\|\left.\left(P_{\epsilon} u^{\epsilon}-u_{0}\right)\right|_{\Omega^{\epsilon}}\right\|_{Z_{\epsilon}} \leq\left\|P_{\epsilon} u^{\epsilon}-u_{0}\right\|_{L^{2}(\Omega)}
$$

e portanto $u^{\epsilon} \stackrel{E}{\rightarrow} u_{0}$ provando que a família $\left\{u^{\epsilon}=L_{\epsilon}^{-1} f^{\epsilon}\right\}_{\epsilon>0}$ é E-relativamente compacta. Mostremos agora que $L_{\epsilon}^{-1} \stackrel{E E}{\rightarrow} L_{0}^{-1}$. Para isto, assuma que $f^{\epsilon} \stackrel{E}{\rightarrow} f_{0}$. Logo, existe uma constante $\mathrm{C}$, independente de $\epsilon$ tal que $\left\|\widetilde{f}^{\epsilon}\right\|_{L^{2}(\Omega)} \leq C$. Seguindo (3.43) e (3.44), concluimos pelo Teorema 3.4.1 que $L_{\epsilon}^{-1} f^{\epsilon} \rightarrow L_{0}^{-1} f_{0}$.

Sabe-se que o espectro de $L_{\epsilon}$ ou $L_{0}$ consiste somente de auto-valores isolados. Consideraremos um autovalor isolado $\lambda_{0} \in \sigma\left(L_{0}\right)$ e associaremos seu autoespaço generalizado $W\left(\lambda_{0}, L_{0}\right)=Q\left(\lambda_{0}, L_{0}\right) Z_{0}$, onde

$$
Q\left(\lambda_{0}, L_{0}\right)=\frac{1}{2 \pi i} \int_{S_{\delta}}\left(\xi I-L_{0}\right)^{-1} d \xi,
$$

$S_{\delta}=\left\{\xi \in \mathbb{C} ;\left|\xi-\lambda_{0}\right|=\delta\right\}$ e $\delta$ é tal que não exista nenhum outro ponto de $\sigma\left(L_{0}\right)$ no disco $\left\{\xi \in \mathbb{C} ;\left|\xi-\lambda_{0}\right| \leq \delta\right\}$. Logo segue do Lema 1.2.3 existe $\epsilon_{0}>0$ tal que $S_{\delta} \subset \rho\left(L_{\epsilon}\right)$ para todo $\epsilon \in\left(0, \epsilon_{0}\right)$. Assim, podemos denotar por $W\left(\lambda_{0}, L_{\epsilon}\right)=Q\left(\lambda_{0}, L_{\epsilon}\right) Z_{\epsilon}$ onde

$$
Q\left(\lambda_{0}, L_{\epsilon}\right)=\frac{1}{2 \pi i} \int_{S_{\delta}}\left(\xi I-L_{\epsilon}\right)^{-1} d \xi
$$

Do corolário anterior, concluimos que os operadores $L_{\epsilon}$ satisfazem a seguinte hipótese

$$
L_{\epsilon} \text { é fechado, tem resolvente compacto, } 0 \in \rho\left(L_{\epsilon}\right) \text {, e } L_{\epsilon}^{-1} \stackrel{C C}{\longrightarrow} L_{0}^{-1} \text {. }
$$

Consequentemente, segue do Teorema 1.2.1 as seguintes afirmações a respeito da convergência do espectro de $L_{\epsilon}$.

(i) Para qualquer $\lambda_{0} \in \sigma\left(L_{0}\right)$, existe uma sequência $\lambda_{\epsilon} \in \sigma\left(L_{\epsilon}\right)$, tal que $\lambda_{\epsilon} \stackrel{\epsilon \rightarrow 0}{\rightarrow} \lambda_{0}$.

(ii) Se $\lambda_{\epsilon} \rightarrow \lambda_{0}$, com $\lambda_{\epsilon} \in \sigma\left(L_{\epsilon}\right)$, então $\lambda_{0} \in \sigma\left(L_{0}\right)$.

(iii) Existe $\epsilon_{0}>0$ tal que $\operatorname{dim} W\left(\lambda_{0}, L_{\epsilon}\right)=\operatorname{dim} W\left(\lambda_{0}, L_{0}\right)$ para todo $0<\epsilon \leq \epsilon_{0}$.

(iv) Para cada $u \in W\left(\lambda_{0}, L_{0}\right)$, existe uma sequência $u^{\epsilon} \in W\left(\lambda_{0}, L_{\epsilon}\right)$, tal que $u^{\epsilon} \stackrel{E}{\rightarrow} u$.

(v) Se $u^{\epsilon} \in W\left(\lambda_{0}, L_{\epsilon}\right)$ satisfaz $\left\|u^{\epsilon}\right\|_{Z_{\epsilon}}=1$, então $\left\{u^{\epsilon}\right\}$ tem uma subsequência E-convergente e o seu limite pertence a $W\left(\lambda_{0}, L_{0}\right)$. 
No que segue, estenderemos a convergência compacta para $\left(\lambda-L_{\epsilon}\right)^{-1} \stackrel{E E}{\rightarrow}\left(\lambda-L_{0}\right)^{-1}$ com $\lambda$ em um setor que está contido no resolvente de todos os $L_{\epsilon}$.

Considere os seguintes operadores $M_{\epsilon}, \widetilde{M}_{\epsilon}: L^{p}\left(\Omega^{\epsilon}\right) \mapsto L^{p}(0,1), 1 \leq p \leq \infty$, dados por

$$
\begin{gathered}
\left(M_{\epsilon} f^{\epsilon}\right)\left(x_{1}\right)=\frac{L}{\left|Y^{*}\right|} \int_{0}^{g\left(\frac{x_{1}}{\epsilon}\right)} f^{\epsilon}\left(x_{1}, x_{2}\right) d x_{2}, x_{1} \in(0,1), \\
\left(\widetilde{M}_{\epsilon} f^{\epsilon}\right)\left(x_{1}\right)=\frac{1}{g\left(x_{1} / \epsilon\right)} \int_{0}^{g\left(\frac{x_{1}}{\epsilon}\right)} f^{\epsilon}\left(x_{1}, x_{2}\right) d x_{2}, \quad x_{1} \in(0,1)
\end{gathered}
$$

Segue do Teorema de Fubini-Tonelli e da Desigualdade de Hölder que $M_{\epsilon}, \widetilde{M}_{\epsilon}$ estão bem definidos, são lineares com

$$
\left\|M_{\epsilon} f^{\epsilon}\right\|_{L^{p}(0,1)},\left\|\widetilde{M}_{\epsilon} f^{\epsilon}\right\|_{L^{p}(0,1)} \leq C\left\|f^{\epsilon}\right\|_{L^{p}\left(\Omega^{\epsilon}\right)} .
$$

onde $C>0$ é uma constante independente de $\epsilon$. Em particular existe uma constante $C>0$ independente de $\epsilon$ tal que

$$
\left\|M_{\epsilon} f^{\epsilon}\right\|_{Z_{0}},\left\|M_{\epsilon} f^{\epsilon}\right\|_{Z_{0}} \leq C\left\|f^{\epsilon}\right\|_{Z_{\epsilon}} .
$$

Com isto temos o seguinte

Lema 3.5.1. Seja $\left\{f^{\epsilon}\right\}_{0<\epsilon \leq 1}, f^{\epsilon} \in Z_{\epsilon}$ uma sequência e suponha que $\left\|f^{\epsilon}\right\|_{Z_{\epsilon}} \leq C$, para algum $C$ indepenpdente de $\epsilon$. Então para cada sequência $\left\{\epsilon_{n}\right\}$ tendendo a zero, existe uma subsequência desta (que denotaremos também por $\epsilon_{n}$ ) tal que

$$
\left\|L_{\epsilon_{n}}^{-1} f^{\epsilon_{n}}-E_{\epsilon_{n}} L_{0}^{-1} M_{\epsilon_{n}} f^{\epsilon_{n}}\right\|_{Z_{\epsilon_{n}}} \rightarrow 0 \text { quando } n \rightarrow \infty .
$$

Consequentemente,

$$
\left\|L_{\epsilon}^{-1}-E_{\epsilon} L_{0}^{-1} M_{\epsilon}\right\|_{\mathcal{L}\left(Z_{\epsilon}\right)} \stackrel{\epsilon \rightarrow 0}{\rightarrow} 0
$$

Em particular, se $f^{\epsilon} \stackrel{E}{\rightarrow} f$ quando $\epsilon \rightarrow 0$, então

$$
\left\|L_{\epsilon}^{-1} f^{\epsilon}-E_{\epsilon} L_{0}^{-1} M_{\epsilon} f^{\epsilon}\right\|_{Z_{\epsilon}} \stackrel{\epsilon \rightarrow 0}{\rightarrow} 0 .
$$

Prova: Já temos $M_{\epsilon} f^{\epsilon} \rightarrow f_{0}$ em $L^{2}(0,1)$ onde $f_{0}$ é como no Teorema 3.4.1. Como $L_{0}^{-1} \in$ $\mathcal{L}\left(L^{2}(0,1)\right)$ é compacto e auto adjunto, seque que $L_{0}^{-1} M_{\epsilon} f^{\epsilon} \rightarrow L_{0}^{-1} f_{0}$ em $L^{2}(0,1)$ e portanto,

$$
\left\|E_{\epsilon} L_{0}^{-1} M_{\epsilon} f^{\epsilon}-E_{\epsilon} L_{0}^{-1} f_{0}\right\|_{Z_{\epsilon}} \stackrel{\epsilon \rightarrow 0}{\rightarrow} 0
$$

Ainda do Teorema 3.4.1, $P_{\epsilon}\left(L_{\epsilon}^{-1} f^{\epsilon}\right) \rightarrow L_{0}^{-1} f_{0}$ em $L^{2}(\Omega)$ e como $\left.P_{\epsilon}\left(L_{\epsilon}^{-1} f^{\epsilon}\right)\right|_{\Omega^{\epsilon}}=L_{\epsilon}^{-1} f^{\epsilon}$ segue que

$$
\left\|L_{\epsilon}^{-1} f^{\epsilon}-E_{\epsilon} L_{0}^{-1} f_{0}\right\|_{Z_{\epsilon}} \stackrel{\epsilon \rightarrow 0}{\rightarrow} 0
$$


Assim, de (3.72) e (3.73) segue o lema

Como consequência do lema anterior, estabelecemos o seguinte corolário.

Corolário 3.5.2. Seja $f \in L^{2}(0,1)$ e considere a sequência $\left\{E_{\epsilon} f\right\}_{0<\epsilon \leq 1}$. Então

$$
\left\|L_{\epsilon} E_{\epsilon} f-E_{\epsilon} L_{0}^{-1} f\right\|_{Z_{\epsilon}} \stackrel{\epsilon \rightarrow 0}{\rightarrow} 0 .
$$

Prova: Como $E_{\epsilon} f \stackrel{E}{\rightarrow} f$ quando $\epsilon \rightarrow 0$ segue do Lema 3.5.1 que

$$
\left\|L_{\epsilon} E_{\epsilon} f-E_{\epsilon} L_{0}^{-1} M_{\epsilon} E_{\epsilon} f\right\|_{Z_{\epsilon}} \stackrel{\epsilon \rightarrow 0}{\rightarrow} 0 .
$$

e portanto basta mostramos que

$$
\left\|E_{\epsilon} L_{0}^{-1} M_{\epsilon} E_{\epsilon} f-E_{\epsilon} L_{0}^{-1} f\right\|_{Z_{\epsilon}} \stackrel{\epsilon \rightarrow 0}{\rightarrow} 0 .
$$

Como $\widetilde{M}_{\epsilon} E_{\epsilon}=I_{L^{2}(0,1)}$ segue que o limite acima equivale a

$$
\left\|E_{\epsilon} L_{0}^{-1}\left(M_{\epsilon}-\widetilde{M}_{\epsilon}\right) E_{\epsilon} f\right\|_{Z_{\epsilon}} \stackrel{\epsilon \rightarrow 0}{\rightarrow} 0
$$

Continuando temos

$$
\left[\left(M_{\epsilon}-\widetilde{M}_{\epsilon}\right) E_{\epsilon}\right] f\left(x_{1}\right)=\frac{L}{\left|Y^{*}\right|} f\left(x_{1}\right) g\left(\frac{x_{1}}{\epsilon}\right)-f\left(x_{1}\right),
$$

e portanto

$$
\left(M_{\epsilon}-\widetilde{M}_{\epsilon}\right) E_{\epsilon} f \rightarrow 0 \text { em } L^{2}(0,1) \text { quando } \epsilon \rightarrow 0 .
$$

Logo $L_{0}^{-1}\left(M_{\epsilon}-\widetilde{M}_{\epsilon}\right) E_{\epsilon} f \rightarrow 0$ em $L^{2}(0,1)$ quando $\epsilon \rightarrow 0$ e o resultado segue.

Note que a imagem numérica dos operadores $-L_{\epsilon}$ estão contidas em $(-\infty,-1]$ para todo $\epsilon>0$. Assim, concluimos do Teorema 0.0.4 que existem $M>0$ e $\frac{\pi}{2}<\phi<\pi$, independente de $\epsilon$ tal que

$$
\left\|\left(\mu+L_{\epsilon}\right)^{-1}\right\|_{\mathcal{L}\left(Z_{\epsilon}\right)} \leq \frac{M}{|\mu+1|} \forall \mu \in \Sigma_{-1, \phi}
$$

onde $\Sigma_{-1, \phi}=\{\mu \in \mathbb{C}: 0<|\arg (\mu+1)| \leq \phi\}$. Aqui, substituimos $Z_{\epsilon}$ por $Z_{0}$ quando $\epsilon=0$. Consequentemente os operadores $L_{\epsilon}$ são setoriais com estimativa da norma do resolvente $\left(\mu-L_{\epsilon}\right)$ no setor $\mathbb{C}-\Sigma_{1, \pi-\phi}$.

Para se obter a $E E$-convergência do resolvente para $\lambda$ diferente de zero, faremos uso do seguinte

Lema 3.5.2. Para todo $\lambda \in \rho\left(L_{\epsilon}\right)$ temos

$$
\left(\lambda-L_{\epsilon}\right)^{-1} E_{\epsilon}-E_{\epsilon}\left(\lambda-L_{0}\right)^{-1}=\left[I-\lambda\left(\lambda-L_{\epsilon}\right)^{-1}\right]\left[L_{\epsilon}^{-1}-E_{\epsilon} L_{0}^{-1} \widetilde{M}_{\epsilon}\right]\left[E_{\epsilon} L_{0}\left(\lambda-L_{0}\right)^{-1}\right] .
$$


Prova: Sabendo que $I-\lambda\left(\lambda-L_{\epsilon}\right)^{-1}=-L_{\epsilon}\left(\lambda-L_{\epsilon}\right)^{-1}$ e $\widetilde{M}_{\epsilon} E_{\epsilon}=I$ segue que

$$
\begin{aligned}
{[I} & \left.-\lambda\left(\lambda-L_{\epsilon}\right)^{-1}\right]\left[L_{\epsilon}^{-1}-E_{\epsilon} L_{0}^{-1} \widetilde{M}_{\epsilon}\right] E_{\epsilon} L_{0}\left(\lambda-L_{0}\right)^{-1} \\
& =-L_{\epsilon}\left(\lambda-L_{\epsilon}\right)^{-1}\left[L_{\epsilon}^{-1}-E_{\epsilon} L_{0}^{-1} \widetilde{M}_{\epsilon}\right] E_{\epsilon} L_{0}\left(\lambda-L_{0}\right)^{-1} \\
& =\left[-\left(\lambda-L_{\epsilon}\right)^{-1}+L_{\epsilon}\left(\lambda-L_{\epsilon}\right)^{-1} E_{\epsilon} L_{0}^{-1} \widetilde{M}_{\epsilon}\right] E_{\epsilon} L_{0}\left(\lambda-L_{0}\right)^{-1} \\
& =-\left(\lambda-L_{\epsilon}\right)^{-1} E_{\epsilon} L_{0}\left(\lambda-L_{0}\right)^{-1}+L_{\epsilon}\left(\lambda-L_{\epsilon}\right)^{-1} E_{\epsilon} L_{0}^{-1} \widetilde{M}_{\epsilon} E_{\epsilon} L_{0}\left(\lambda-L_{0}\right)^{-1} \\
& =-\left(\lambda-L_{\epsilon}\right)^{-1} E_{\epsilon} L_{0}\left(\lambda-L_{0}\right)^{-1}+L_{\epsilon}\left(\lambda-L_{\epsilon}\right)^{-1} E_{\epsilon}\left(\lambda-L_{0}\right)^{-1} \\
& =\left(\lambda-L_{\epsilon}\right)^{-1} E_{\epsilon}\left[I-\lambda\left(\lambda-L_{0}\right)^{-1}\right]-\left[I-\lambda\left(\lambda-L_{\epsilon}\right)^{-1}\right] E_{\epsilon}\left(\lambda-L_{0}\right)^{-1} \\
& =\left(\lambda-L_{\epsilon}\right)^{-1} E_{\epsilon}-\left(\lambda-L_{\epsilon}\right)^{-1} E_{\epsilon} \lambda\left(\lambda-L_{0}\right)^{-1}-E_{\epsilon}\left(\lambda-L_{0}\right)^{-1}+\lambda\left(\lambda-L_{\epsilon}\right)^{-1} E_{\epsilon}\left(\lambda-L_{0}\right)^{-1} \\
& =\left(\lambda-L_{\epsilon}\right)^{-1} E_{\epsilon}-E_{\epsilon}\left(\lambda-L_{0}\right)^{-1} .
\end{aligned}
$$

e isto conclui o lema

Usando (3.74) obtemos

$$
\begin{gathered}
\left\|I-\lambda\left(\lambda-L_{\epsilon}\right)^{-1}\right\|_{\mathcal{L}\left(Z_{\epsilon}\right)} \leq 1+\frac{|\lambda|}{|\lambda+1|} M \\
\left\|E_{\epsilon} L_{0}\left(\lambda-L_{0}\right)^{-1} M_{\epsilon}\right\|_{\mathcal{L}\left(Z_{\epsilon}\right)} \leq\left\|E_{\epsilon}\right\|\left(1+\frac{|\lambda|}{|\lambda+1|} M\right) .
\end{gathered}
$$

Consequentemente obtemos o seguinte

Corolário 3.5.3. Para todo $\lambda \in \mathbb{C}-\Sigma_{1, \pi-\phi}$ temos $\left(\lambda-L_{\epsilon}\right)^{-1} \stackrel{E E}{\rightarrow}\left(\lambda-L_{0}\right)^{-1}$

Prova: Suponha que $f^{\epsilon} \stackrel{E}{\rightarrow} f$. Segue do Lema 3.5.2, do Corolário 3.5.2 e de (3.75) que

$$
\left\|\left[\left(\lambda-L_{\epsilon}\right)^{-1} E_{\epsilon}-E_{\epsilon}\left(\lambda-L_{0}\right)^{-1}\right] f\right\|_{Z_{\epsilon}} \stackrel{\epsilon \rightarrow 0}{\rightarrow} 0 .
$$

Como

$$
\left(\lambda-L_{\epsilon}\right)^{-1} f^{\epsilon}-E_{\epsilon}\left(\lambda-L_{0}\right)^{-1} f=\left(\lambda-L_{\epsilon}\right)^{-1}\left(f^{\epsilon}-E_{\epsilon} f\right)+\left[\left(\lambda-L_{\epsilon}\right)^{-1} E_{\epsilon}-E_{\epsilon}\left(\lambda-L_{0}\right)^{-1}\right] f
$$

e $\left\|\left(\lambda-L_{\epsilon}\right)^{-1}\right\|_{\mathcal{L}\left(Z_{\epsilon}\right)}$ é uniformemente limitada em $\epsilon$, o resultado segue.

\subsection{Convergência dos Semigrupos}

Nesta seção estudamos algumas propriedades dos semigrupos lineares gerados pelos operadores $L_{\epsilon}$ e $L_{0}$ apresentados na seção anterior.

Segue do Corolário 3.5.3 que

$$
\left(\mu+L_{\epsilon}\right)^{-1} \stackrel{E E}{\rightarrow}\left(\mu+L_{0}\right)^{-1}, \forall \mu \in \Sigma_{-1, \phi}
$$


e como

$$
\left\|\left(\mu+L_{\epsilon}\right)^{-1}\right\|_{\mathcal{L}\left(Z_{\epsilon}\right)} \leq \frac{M}{|\mu+1|} \forall \mu \in \Sigma_{-1, \phi}
$$

segue do Teorema de Trotter-Kato (Teorema 1.4.4) que existe $0<\theta<\frac{\pi}{2}$ tal que $\forall \epsilon_{0}, \mu \in$ $(0, \infty), \epsilon_{0}<\mu$ temos

$$
\sup _{t \in \Sigma\left(\theta, \epsilon_{0}, \mu\right)}\left\|e^{-L_{\epsilon} t} u_{n}-E_{\epsilon} e^{-L_{0} t} u_{0}\right\|_{Z_{\epsilon}} \stackrel{\epsilon \rightarrow 0}{\rightarrow} 0
$$

sempre que $u_{n} \stackrel{E}{\rightarrow} u_{0}$, onde

$$
\Sigma\left(\theta, \epsilon_{0}, \mu\right)=\left\{z \in \mathbb{C}:|\arg (z)| \leq \theta \text { e } \epsilon_{0} \leq|t| \leq \mu\right\}
$$

isto é, $e^{-L_{\epsilon} t} \stackrel{E E}{\rightarrow} e^{-L_{0} t}$ uniformemente em compactos $\Sigma\left(\theta, \epsilon_{0}, \mu\right)$

Observação 3.6.1. Segue do Teorema 1.3.2 que a familia de semigrupos $\left\{e^{-L_{\epsilon} t}: t \geq 0\right\}$ satistaz

$$
\left\|e^{-L_{\epsilon} t}\right\|_{\mathcal{L}\left(Z_{\epsilon}, Z_{\epsilon}^{\alpha}\right)} \leq M_{\alpha} t^{-\alpha} e^{-1}, \forall t>0 \text { e } 0 \leq \epsilon \leq \epsilon_{0}
$$

No que segue, tentaremos para cada sequência $\left\{f^{\epsilon}\right\} \operatorname{com} f^{\epsilon} \stackrel{E}{\rightarrow} f$ dar uma taxa de convergência como acima.

Dado $r>0$ e $f \in L^{2}(0,1)$, segue do Corolário 3.5.2, do Lema 3.5.2 e de (3.75) que existe uma função $\nu:(0,1) \rightarrow \mathbb{R}^{+}, \nu(\epsilon) \rightarrow 0$ quando $\epsilon \rightarrow 0$, tal que

$$
\left\|\left(\mu+L_{\epsilon}\right)^{-1} E_{\epsilon} f-E_{\epsilon}\left(\mu+L_{0}\right)^{-1} f\right\|_{Z_{\epsilon}} \leq \nu(\epsilon .)
$$

independente de $\mu$ com $\mu \in \Sigma_{-1, \phi} \backslash B(-1, r)$.

Observação 3.6.2. Para cada $\epsilon>0$ temos $D\left(L_{\epsilon}^{\frac{1}{2}}\right):=\operatorname{Im}\left(L_{\epsilon}^{-\frac{1}{2}}\right)=H^{1}\left(\Omega^{\epsilon}\right)$. Isto segue do fato de que o conjunto $H_{N_{\epsilon}}^{1}:=\left\{u \in C^{2}\left(\Omega^{\epsilon}\right): \frac{\partial u}{\partial N}=0\right.$ em $\left.\partial \Omega^{\epsilon}\right\}$ é tal que $H_{N_{\epsilon}}^{1} \subset D\left(L_{\epsilon}\right) \subset D\left(L_{\epsilon}^{\frac{1}{2}}\right)$ e $H_{N_{\epsilon}}^{1}$ é denso em $H^{1}\left(\Omega^{\epsilon}\right)$ com a norma $\|\cdot\|_{H^{1}\left(\Omega^{\epsilon}\right)}$ e esta norma é equivalente a norma derivada da forma bilinear $a_{\epsilon}$ em $H_{N_{\epsilon}}^{1}$.

Para obtermos taxa de convergência, precisaremos do seguinte lema.

Lema 3.6.1. Existem $M>0$ e $\epsilon_{0}>0$ tais que para todo $u \in H^{1}(0,1)$ e $0<\epsilon \leq \epsilon_{0}$,

$$
\left\|E_{\epsilon}\right\|_{Z_{\epsilon}^{\frac{1}{2}}}:=\left\|L_{\epsilon}^{\frac{1}{2}} E_{\epsilon} u\right\|_{Z_{\epsilon}} \leq M\left\|L_{0}^{\frac{1}{2}} u\right\|_{Z_{0}}=: M\|u\|_{Z_{0}^{\frac{1}{2}}}
$$

Prova: Como, de (3.20), existem $M_{1}, \epsilon_{0}>0$ tais que

$$
\left\|V^{\epsilon}\right\|_{L^{\infty}\left(\Omega^{\epsilon}\right)} \leq M_{1}, \forall 0<\epsilon \leq \epsilon_{0}
$$


Disto segue que para todo $u \in H^{1}(0,1)$,

$$
\begin{aligned}
\left\|L_{\epsilon}^{\frac{1}{2}} E_{\epsilon} u\right\|_{Z_{\epsilon}}^{2} & =a_{\epsilon}\left(E_{\epsilon} u, E_{\epsilon} u\right)=\int_{0}^{1} g\left(\frac{x_{1}}{\epsilon}\right)\left(\frac{d u}{d x_{1}}\left(x_{1}\right)\right)^{2} d x_{1}+\int_{\Omega^{\epsilon}} V^{\epsilon}(u)^{2} d x_{1} d x_{2} \\
& \leq g_{1}\left\|\frac{d u}{d x_{1}}\right\|_{L^{2}(0,1)}^{2}+M_{1}\|u\|_{L^{2}(0,1)}^{2} \leq \max \left\{g_{1}, M_{1}\right\}\|u\|_{H^{1}(0,1)}^{2} .
\end{aligned}
$$

Por outro lado, para todo $u \in H^{1}(0,1)$,

$$
\begin{aligned}
\left\|L_{0}^{\frac{1}{2}} u\right\|_{Z_{0}}^{2} & =g_{1}\left\|L_{0}^{\frac{1}{2}} u\right\|_{L^{2}(0,1)}^{2}=g_{1} a_{0}(u, u) \\
& =g_{1} \int_{0}^{1}\left[q_{0}\left(\frac{d u}{d x_{1}}\right)^{2}+V_{0}(u)^{2}\right] d x_{1} \geq g_{1} \int_{0}^{1}\left[q_{0}\left(\frac{d u}{d x_{1}}\right)^{2}+(u)^{2}\right] \\
& \geq g_{1} \min \left\{q_{0}, 1\right\}\|u\|_{H^{1}(0,1)}^{2} .
\end{aligned}
$$

De (3.78) e (3.79) segue que para todo $0<\epsilon \leq \epsilon_{0}, u \in H^{1}(0,1)$ temos

$$
\left\|L_{\epsilon}^{\frac{1}{2}} E_{\epsilon} u\right\|_{Z_{\epsilon}}^{2} \leq \frac{\max \left\{g_{1}, M_{1}\right\}}{g_{1} \min \left\{q_{0}, 1\right\}} g_{1} \min \left\{q_{0}, 1\right\}\|u\|_{H^{1}(0,1)}^{2} \leq \frac{\max \left\{g_{1}, M_{1}\right\}}{g_{1} \min \left\{q_{0}, 1\right\}}\left\|L_{0}^{\frac{1}{2}} u\right\|_{Z_{0}}^{2},
$$

e com isto completamos a prova.

O teorema a seguir é fundamental para estabelecermos taxa de $E E$-convergência dos semigrupos.

Teorema 3.6.1. Suponha que $0 \leq \alpha<\frac{1}{2}$ e $f \in L^{2}(0,1)$. Então existe uma função $\nu_{\alpha}$ : $(0,1] \rightarrow \mathbb{R}^{+}, \nu_{\alpha}(\epsilon) \stackrel{\epsilon \rightarrow 0}{\rightarrow} 0$, tal que

$$
\left\|\left[e^{-L_{\epsilon} t} E_{\epsilon}-E_{\epsilon} e^{-L_{0} t}\right] f\right\|_{Z_{\epsilon}} \leq \nu_{\alpha}(\epsilon) e^{-t} t^{\alpha-1} \forall t>0 .
$$

Prova: Como os operadores $L_{\epsilon}$ são setoriais, temos

$$
e^{\left(-L_{\epsilon}+1\right) t}=\frac{1}{2 \pi i} \int_{\Gamma} e^{(\mu+1) t}\left(\mu+1+L_{\epsilon}-1\right)^{-1} d \mu
$$

onde $\Gamma$ é a fronteira de $\Sigma_{-1, \phi}-B_{r}(-1), 0<r<1$ com $\frac{\pi}{2}<\phi<\pi$ orientada de tal modo que a parte imaginária seja crescente quando $\mu$ percorre $\Gamma$.

Fazendo a mudança de variaveis $\mu+1 \mapsto \mu$ e denotanto $; B_{\epsilon}:=L_{\epsilon}-1$ obtemos

$$
2 \pi\left\|e^{-B_{\epsilon} t} E_{\epsilon} f-E_{\epsilon} e^{-B_{0} t} f\right\|_{Z_{\epsilon}^{\alpha}}=\left\|\int_{\Gamma_{0}} e^{\mu t}\left[\left(\mu+B_{\epsilon}\right)^{-1} E_{\epsilon} f-E_{\epsilon}\left(\mu+B_{0}\right)^{-1} f\right] d \mu\right\|_{Z_{\epsilon}^{\alpha}}
$$

onde $\Gamma_{0}$ é a fronteira de $\Sigma_{0, \phi}-B_{r}(0)$ orientada com a parte imaginária crescente.

Segue de (3.74) que $\left\|\left(\mu+B_{\epsilon}\right)^{-1}\right\|_{\mathcal{L}\left(Z_{\epsilon}\right)} \leq \frac{M}{|\mu|}$ para todo $\mu \in \Gamma_{0}$ e $\epsilon \in[0,1]$. Portanto

$$
\left\|\left(\mu+B_{\epsilon}\right)^{-1} E_{\epsilon} f-E_{\epsilon}\left(\mu+B_{0}\right)^{-1} f\right\|_{Z_{\epsilon}} \leq \frac{M\left\|E_{\epsilon}\right\|}{|\mu|}\|f\|_{L^{2}(0,1)} \leq \frac{M_{1}}{|\mu|}\|f\|_{L^{2}(0,1)} .
$$


Também temos $\left\|B_{\epsilon}\left(\mu+B_{\epsilon}\right)^{-1} E_{\epsilon} f\right\|_{Z_{\epsilon}} \leq(1+M)\left\|E_{\epsilon} f\right\|_{Z_{\epsilon}}$. Segue da desigualdade do momento que

$$
\begin{aligned}
\left\|\left(\mu+B_{\epsilon}\right)^{-1} E_{\epsilon} f\right\|_{Z_{\epsilon}^{\frac{1}{2}}} & \leq\left\|B_{\epsilon}\left(\mu+B_{\epsilon}\right)^{-1} E_{\epsilon} f\right\|_{Z_{\epsilon}}^{\frac{1}{2}}\left\|\left(\mu+B_{\epsilon}\right)^{-1} E_{\epsilon} f\right\|_{Z_{\epsilon}^{1}}^{\frac{1}{2}} \\
& \leq \frac{M^{\frac{1}{2}}}{|\mu|^{\frac{1}{2}}}(1+M)\left\|E_{\epsilon} f\right\|_{Z_{\epsilon}} \leq \frac{M_{2}}{|\mu|^{\frac{1}{2}}}\|f\|_{L^{2}(0,1)} .
\end{aligned}
$$

Segue da Obsevação 3.6.2 e do Lema 3.6.1 que existem $M_{0}>0$ e $\epsilon_{0}>$ tais que para toda $f \in L^{2}(0,1)$ e $0<\epsilon \leq \epsilon_{0}$,

$$
\left\|L_{\epsilon}^{\frac{1}{2}} E_{\epsilon}\left(\mu+B_{0}\right)^{-1} f\right\|_{Z_{\epsilon}} \leq M_{0}\left\|L_{0}^{\frac{1}{2}}\left(\mu+B_{0}\right)^{-1} f\right\|_{Z_{0}} \leq M_{0} \frac{M^{\frac{1}{2}}}{|\mu|^{\frac{1}{2}}}\|f\|_{L^{2}(0,1)} .
$$

Assim, podemos concluir que

$$
\left\|\left(\mu+B_{\epsilon}\right)^{-1} E_{\epsilon} f-E_{\epsilon}\left(\mu+B_{0}\right)^{-1} f\right\|_{Z_{\epsilon}^{\frac{1}{2}}} \leq \frac{M_{2}^{\frac{1}{2}}}{|\mu|^{\frac{1}{2}}}\|f\|_{L^{2}(0,1)} .
$$

Seja $x:=\left(\mu+B_{\epsilon}\right)^{-1} E_{\epsilon} f-E_{\epsilon}\left(\mu+B_{0}\right)^{-1} f$. Segue do Teorema 1.3.3 que $L_{\epsilon}^{\frac{1}{2}}$ também é setorial e positivo. Usando a desigualdade do momento para $L_{\epsilon}^{\frac{1}{2}}$ (com expoente $2 \alpha$ ) obtemos

$$
\|x\|_{Z_{\epsilon}^{\alpha}} \leq M_{3}\|x\|_{Z_{\epsilon}^{\frac{1}{2}}}^{2 \alpha}\|x\|_{Z_{\epsilon}}^{1-2 \alpha}
$$

Usando também as estimativas (3.81),(3.82) e (3.77) obtemos

$$
\left\|\left(\mu+B_{\epsilon}\right)^{-1} E_{\epsilon} f-E_{\epsilon}\left(\mu+B_{0}\right)^{-1} f\right\|_{Z_{\epsilon}^{\alpha}} \leq \frac{M_{4} \nu(\epsilon)^{(1-2 \alpha)}}{|\mu|^{\alpha}} .
$$

Com a mudânça de variáveis $\beta=\mu t$ em (3.80) temos a integral equivalente

$$
\left\|\int_{\Gamma_{0}} e^{\beta}\left[\left(\beta t^{-1}+B_{\epsilon}\right)^{-1} E_{\epsilon} f-E_{\epsilon}\left(\beta t^{-1}+B_{0}\right)^{-1} f\right] \frac{d \beta}{t}\right\|_{Z_{\epsilon}^{\alpha}}
$$

Agora, de (3.83) temos

$$
\begin{aligned}
\left\|\frac{1}{t} \int_{\Gamma_{0}} e^{\beta}\left[\left(\beta t^{-1}+B_{\epsilon}\right)^{-1} E_{\epsilon} f-E_{\epsilon}\left(\beta t^{-1}+B_{0}\right)^{-1} f\right] d \beta\right\|_{Z_{\epsilon}^{\alpha}} & \leq M_{4} t^{\alpha-1} \nu(\epsilon)^{1-2 \alpha} \int_{\Gamma_{0}} \frac{\left|e^{\beta}\right|}{|\beta|^{\alpha}} d|\beta| .
\end{aligned}
$$

e portanto

$$
\left\|e^{-B_{\epsilon} t} E_{\epsilon} f-E_{\epsilon} e^{-B_{0} t} f\right\|_{Z_{\epsilon}^{\alpha}} \leq M_{5} t^{1-\alpha} \nu(\epsilon)^{(1-2 \alpha)} \text { para } t>0
$$


Logo, $\forall \alpha \in\left[0, \frac{1}{2}\right)$, existe uma função denotada por $\nu_{\alpha}:(0,1] \rightarrow \mathbb{R}^{+} \operatorname{com} \nu(\epsilon) \stackrel{\epsilon \rightarrow 0}{\rightarrow} 0$, tal que

$$
\left\|e^{-L_{\epsilon} t} E_{\epsilon} f-E_{\epsilon} e^{-L_{0} t} f\right\|_{Z_{\epsilon}^{\alpha}} \leq \nu_{\alpha}(\epsilon) e^{-t} t^{1-\alpha} \text { para } t>0 .
$$

Como corolário, obtemos a taxa de $E E$-convergência dos semigrupos.

Corolário 3.6.1. Suponha que $0 \leq \alpha<\frac{1}{2}$ e $u_{\epsilon} \stackrel{E}{\rightarrow} u$. Então existe uma função $\nu:(0,1] \rightarrow$ $\mathbb{R}^{+}, \nu(\epsilon) \stackrel{\epsilon \rightarrow 0}{\rightarrow} 0$, tal que

$$
\left\|e^{-L_{\epsilon} t} u_{\epsilon}-E_{\epsilon} e^{-L_{0} t} u\right\|_{Z_{\epsilon}^{\alpha}} \leq \nu(\epsilon) e^{-t} t^{-\alpha} \forall t>0
$$

Prova: Note que

$$
e^{-L_{\epsilon} t} u_{\epsilon}-E_{\epsilon} e^{-L_{0} t} u_{0}=e^{-L_{\epsilon} t}\left(u_{\epsilon}-E_{\epsilon} u_{0}\right)+\left[e^{-L_{\epsilon} t} E_{\epsilon} u-E_{\epsilon} e^{-L_{0} t} u\right]
$$

$\log \mathrm{O}$

$$
\left\|e^{-L_{\epsilon} t} u_{\epsilon}-E_{\epsilon} e^{-L_{0} t} u_{0}\right\|_{Z_{\epsilon}^{\alpha}} \leq M_{\alpha} t^{-\alpha} e^{-t}\left\|u_{\epsilon}-E_{\epsilon} u_{0}\right\|_{Z_{\epsilon}^{\alpha}}+\nu_{\alpha}(\epsilon) t^{\alpha-1} e^{-t}
$$

como para todo $t>0$ temos $t^{\alpha-1} \leq t^{-\alpha}$ (pois $0 \leq \alpha<\frac{1}{2}$ ) segue que

$$
\left\|e^{-L_{\epsilon} t} u_{\epsilon}-E_{\epsilon} e^{-L_{0} t} u_{0}\right\|_{Z_{\epsilon}^{\alpha}} \leq\left[M_{\alpha}\left\|u_{\epsilon}-E_{\epsilon} u_{0}\right\|_{Z_{\epsilon}^{\alpha}}+\nu_{\alpha}(\epsilon)\right] t^{-\alpha} e^{-t}
$$

Assim, tomando $\nu(\epsilon):=M_{\alpha}\left\|u_{\epsilon}-E_{\epsilon} u_{0}\right\|_{Z_{\epsilon}^{\alpha}}+\nu_{\alpha}(\epsilon)$ obtemos o resultado. $\square$ 


\section{Capítulo 4}

\section{Apêndice: estimativa do erro}

Neste capítulo estamos interessados em estimar o erro que se comete na aproximação de $u^{\epsilon}$ pela sua expansão assintótica

$$
u^{\epsilon}\left(x_{1}, x_{2}\right)=u_{0}\left(x_{1}, x_{2}, \frac{x_{1}}{\epsilon}, \frac{x_{2}}{\epsilon}\right)+\epsilon u_{1}\left(x_{1}, x_{2}, \frac{x_{1}}{\epsilon}, \frac{x_{2}}{\epsilon}\right)+\epsilon^{2} u_{2}\left(x_{1}, x_{2}, \frac{x_{1}}{\epsilon}, \frac{x_{2}}{\epsilon}\right)+\cdots
$$

Seja $f: \mathbb{R} \rightarrow \mathbb{R}$ uma função L-periódica e de classe $C^{1}$ com

$$
0 \leq g_{1} \leq g(x) \leq g_{0} \forall x \in \mathbb{R}
$$

onde $g_{1}=\max \{g(x) ; x \in \mathbb{R}\}$ e $g_{0}=\min \{g(x) ; x \in \mathbb{R}\}$

Considere o seguinte conjunto

$$
R^{\epsilon}=\left\{(x, y) \in \mathbb{R}^{2} ; 0<x<1 \text { e } 0<y<\epsilon g(x / \epsilon)\right\}
$$

onde $\epsilon>0$ é arbitrário.

Considere o seguinte problema

$$
\left\{\begin{array}{l}
-\Delta u^{\epsilon}+u^{\epsilon}=f\left(u^{\epsilon}\right) \text { em } R^{\epsilon} \\
\frac{\partial u^{\epsilon}}{\partial \nu^{\epsilon}} \text { em } \partial R^{\epsilon}
\end{array}\right.
$$

onde $\nu^{\epsilon}$ é o vetor normal unitário exterior a $\partial R^{\epsilon}$.

Relembramos que $u_{0}$ satisfaz o seguinte problema

$$
\left\{\begin{array}{c}
-q_{0} u_{0}^{\prime \prime}+u_{0}=f_{0}, \text { em }(0,1) \\
u_{0}^{\prime}(0)=u_{0}^{\prime}(1)=0
\end{array}\right.
$$

onde

$$
q_{0}=\frac{1}{\left|Y^{*}\right|} \int_{Y^{*}}\{1-\partial X(y, z)\} d y d z
$$


é chamado o coeficiente de homogeneização, $f_{0}$ é como no Teorema 3.4.1, $Y^{*}=\{(y, z) \in$ $\left.\mathbb{R}^{2}: 0<y<L, 0<z<g(y)\right\}$ e $X \in H^{1}\left(Y^{*}\right)$ é solução fraca do problema auxiliar

$$
\left\{\begin{array}{l}
-\Delta_{y, z} X(y, z)=0, \text { em } Y^{*} \\
\frac{\partial X}{\partial N}(y, g(y))=N_{1} \text { em } B_{1} \\
\frac{\partial X}{\partial N}(y, z)=0 \text { em } B_{2} \\
X(0, z)=X(L, z) z \in(0, g(0))
\end{array}\right.
$$

onde $N=\left(N_{1}, N_{2}\right)$ é o campo de vetores normais unitários, $B_{1}=\left\{(y, g(y)) \in \mathbb{R}^{2} \mid 0<y<L\right\}$ é o topo da fronteira $\partial Y^{*}$ e $B_{2}=\left\{(y, 0) \in \mathbb{R}^{2} \mid 0<y<L\right\}$ é a parte inferior de $\partial Y^{*}$.

Sabemos que a convergência $P_{\epsilon} u^{\epsilon} \rightarrow u_{0}$ obtida no capítulo anterior é fraca em $H^{1}(\Omega)$ com a norma $\||\cdot|||$ (definida na próxima seção). Em geral esta convergência é a melhor nestes espaços pois a convergência só pode ser forte em $H^{1}(\Omega)$ se $g$ for constante. Neste capítulo apresentaremos um corretor $\kappa^{\epsilon} \in H^{1}\left(R^{\epsilon}\right), \kappa^{\epsilon}=o(\epsilon)$ em $L^{2}\left(R^{\epsilon}\right)$, tal que

$$
\left\|\left|u^{\epsilon}-u_{0}-\kappa^{\epsilon}\right|\right\|_{H^{1}\left(R^{\epsilon}\right)} \rightarrow 0, \text { quando } \epsilon \rightarrow 0 .
$$

Adicionalmente, comentaremos brevemente sobre um corretor $\mathcal{W}^{\epsilon} \in H^{1}\left(R^{\epsilon}\right)$, tal que

$$
\left\|\left|u^{\epsilon}-\mathcal{W}^{\epsilon}\right|\right\|_{H^{1}\left(R^{\epsilon}\right)} \leq K \epsilon^{1 / 2},
$$

para algum $K>0$ independente de $\epsilon$.

\subsection{Preliminares}

Considere a seguinte medida

$$
\rho_{\epsilon}(\mathcal{O})=\epsilon^{-1}|\mathcal{O}|
$$

onde $|\mathcal{O}|$ é a medida de Lebesgue usual de $\mathbb{R}^{2}$. Introduzimos o espaço de Lebesgue $L^{2}\left(R^{\epsilon} ; \rho_{\epsilon}\right)$ e o espaço de Sobolev $H^{2}\left(R^{\epsilon} ; \rho_{\epsilon}\right)$. As normas destes espaços serão denotadas por $\||\cdot|\|_{L^{2}\left(R^{\epsilon} ; \rho_{\epsilon}\right)}$ e $\||\cdot|\|_{H^{2}\left(R^{\epsilon} ; \rho_{\epsilon}\right)}$ respectivamente, pela introdução dos seguintes produtos internos.

$$
(u, v)_{\epsilon}=\int_{R^{\epsilon}} u v d x, \forall u, v \in L^{2}\left(R^{\epsilon} ; \rho_{\epsilon}\right)
$$

$\mathrm{e}$

$$
a_{\epsilon}(u, v)=\epsilon^{-1} \int_{R^{\epsilon}}\{\nabla u \cdot \nabla v+u v\} d x, \forall u, v \in H^{2}\left(R^{\epsilon} ; \rho_{\epsilon}\right)
$$

respectivamente. 
A formulação variacional de (4.2) é: encontrar $\omega^{\epsilon} \in H^{1}\left(R^{\epsilon}\right)$ tal que

$$
\int_{R^{\epsilon}}\left\{\nabla \omega^{\epsilon} \cdot \nabla \varphi+\omega^{\epsilon} \varphi\right\} d x_{1} d x_{2}=\int_{R^{\epsilon}} f^{\epsilon} \varphi d x_{1} d x_{2}, \forall \varphi \in H^{1}\left(R^{\epsilon}\right)
$$

que é equivalente a encontrar $\omega^{\epsilon} \in H^{1}\left(R^{\epsilon} ; \rho_{\epsilon}\right)$ tal que

$$
a_{\epsilon}\left(\varphi, \omega^{\epsilon}\right)=\left(\varphi, f^{\epsilon}\right)_{\epsilon}, \forall \varphi \in H^{1}\left(R^{\epsilon} ; \rho_{\epsilon}\right) .
$$

Note que as soluções $\omega^{\epsilon}$ satisfazem a priori uma estimativa uniforme em $\epsilon$. De fato, tomando $\varphi=\omega^{\epsilon}$ em (4.4) e (4.5), obtemos

$$
\begin{aligned}
\left\|\nabla \omega^{\epsilon}\right\|_{L^{2}\left(R^{\epsilon}\right)}^{2}+\left\|\omega^{\epsilon}\right\|_{L^{2}\left(R^{\epsilon}\right)}^{2} & \leq\left\|f^{\epsilon}\right\|_{L^{2}\left(R^{\epsilon}\right)}^{2}\left\|\omega^{\epsilon}\right\|_{L^{2}\left(R^{\epsilon}\right)} \\
\|\| \nabla \omega^{\epsilon}\left|\left\|_{L^{2}\left(R^{\epsilon}\right)}^{2}+\right\|\right| \omega^{\epsilon}||_{L^{2}\left(R^{\epsilon}\right)}^{2} & \leq\|\| f^{\epsilon}\left|\left\|_ { L ^ { 2 } ( R ^ { \epsilon } ) } ^ { 2 } \left|\left\|\omega^{\epsilon} \mid\right\|_{L^{2}\left(R^{\epsilon}\right)}\right.\right.\right.
\end{aligned}
$$

Com o objetivo de avaliar o comportamento limite de $a_{\epsilon}\left(\omega^{\epsilon}, \omega^{\epsilon}\right)$ quando $\epsilon \rightarrow 0$, consideramos a forma sesquilinear $a_{0}$ em $H^{1}(0,1)$ dado por

$$
a_{0}(u, v)=\widehat{g} \int_{0}^{1}\left\{\frac{d u}{d x} \frac{d v}{d x}+u v\right\} d x, \forall u, v \in H^{1}(0,1),
$$

onde $\widehat{g}=M_{Y^{*}}(g)=\frac{1}{L} \int_{0}^{L} g(s) d s$.

Em $L^{2}(0,1)$ consideramos o produto interno $(\cdot, \cdot)_{0}$, dado por

$$
(u, v)_{0}=\widehat{g} \int_{0}^{1} u v d x, \forall u, v \in L^{2}(0,1) .
$$

Note que $\widehat{g}$ está relacionada com o período L e a medida da célula representativa $Y^{*}$ pela expressão

$$
L \widehat{g}=\left|Y^{*}\right|
$$

Na Seção 2 do Capítulo 2 obtemos através do método da múltipla escala a seguinte expanção formal

$$
u^{\epsilon}\left(x_{1}, x_{2}\right)=u_{0}\left(x_{1}, x_{2}, \frac{x_{1}}{\epsilon}, \frac{x_{2}}{\epsilon}\right)+\epsilon u_{1}\left(x_{1}, x_{2}, \frac{x_{1}}{\epsilon}, \frac{x_{2}}{\epsilon}\right)+\epsilon^{2} u_{2}\left(x_{1}, x_{2}, \frac{x_{1}}{\epsilon}, \frac{x_{2}}{\epsilon}\right)+\cdots
$$

Além disso obtemos

$$
\left\{\begin{array}{l}
-\Delta_{y, z} u_{2}(x, y, z)=f(x)-u_{0}(x)+\left(1-2 \partial_{y} X(y, z)\right) \frac{d^{2} u_{0}}{d x^{2}}(x) \text { em } Y^{*} \\
\frac{\partial u_{2}}{\partial N}(x, y, g(y))=-\frac{g^{\prime}(y)}{\sqrt{1+g^{\prime}(y)^{2}}} X(y, g(y)) \frac{d^{2} u_{0}}{d x^{2}} \text { em } B_{1} \\
\frac{\partial u_{2}}{\partial N}(x, y, 0)=0 \text { em } B_{2} \\
u_{2}(x, \cdot, z) \text { L-periódica }
\end{array}\right.
$$




$$
\omega_{1}(x, y, z)=-X(y, z) \frac{d u_{0}}{d x}(x)
$$

e

$$
\left\{\begin{array}{c}
-q_{0} \frac{d^{2} u_{0}}{d x^{2}}(x)+u_{0}(x)=f(x), x \in(0,1) \\
u_{0}^{\prime}(0)=u_{0}^{\prime}(1)=0
\end{array}\right.
$$

onde

$$
q_{0}=\frac{1}{\left|Y^{*}\right|} \int_{Y^{*}}\left\{1-\partial_{y} X(y, z)\right\} d y d z .
$$

Aplicando (4.12) a (4.10) obtemos

$$
\left\{\begin{array}{l}
-\operatorname{div}_{y, z}\left(\nabla_{y, z} u_{2}(x, y, z)-\frac{d^{2} u_{0}}{d x^{2}}(x)\left(\begin{array}{c}
X(y, z) \\
0
\end{array}\right)\right)=\left(1-q_{0}-\partial_{y} X(y, z)\right) \frac{d^{2} u_{0}}{d x^{2}}(x) \text { em } Y^{*} \\
\frac{\partial u_{2}}{\partial N}(x, y, g(y))=-\frac{g^{\prime}(y)}{\sqrt{1+g^{\prime}(y)^{2}}} X(y, g(y)) \frac{d^{2} u_{0}}{d x^{2}} \text { em } B_{1} \\
\frac{\partial u_{2}}{\partial N}(x, y, 0)=0 \text { em } B_{2} \\
u_{2}(x, \cdot, z) \text { L-periódica }
\end{array}\right.
$$

O problema (4.14) sugere que procuremos $u_{2}$ da forma

$$
u_{2}(x, y, z)=\theta(y, z) \frac{d^{2} u_{0}}{d x^{2}}(x), x \in(0,1) \text { e }(y, z) \in Y^{*}
$$

onde $\theta$ é solução do problema auxiliar

$$
\left\{\begin{array}{l}
-\operatorname{div}_{y, z}\left(\nabla_{y, z} \theta(y, z)-\left(\begin{array}{c}
X(y, z) \\
0
\end{array}\right)\right)=1-q_{0}-\partial_{y} X(y, z) \text { em } Y^{*} \\
\left(\nabla_{y, z} \theta(y, z)-\left(\begin{array}{c}
X(y, z) \\
0
\end{array}\right)\right) \cdot N=0 \text { em } B_{1} \cup B_{2} \\
\theta(0, z)=\theta(L, z) z \in(0, g(0)) .
\end{array}\right.
$$

Assim, usando (4.11) e (4.15) introduzimos a seguinte expanção assintótica

$$
u^{\epsilon}\left(x_{1}, x_{2}\right)=u_{0}\left(x_{1}\right)-\epsilon X\left(\frac{x_{1}}{\epsilon}, \frac{x_{2}}{\epsilon}\right) \frac{d u_{0}}{d x}\left(x_{1}\right)+\epsilon^{2} \theta\left(\frac{x_{1}}{\epsilon}, \frac{x_{2}}{\epsilon}\right) \frac{d^{2} u_{0}}{d x^{2}}\left(x_{1}\right)+\cdots
$$

Esta expanção será essencial para o que segue. 
Observação 4.1.1. As funções $X$ e $\theta$ são originalmente definidas em $Y^{*}$, mas consideraremos estas funções definidas no domínio fino $R^{\epsilon}$. Usaremos a periodicidade destas funções em y para estender para o conjunto

$$
Y=\left\{(y, z) \in \mathbb{R}^{2} \mid y \in \mathbb{R}, 0<z<g(y)\right\}
$$

e então faremos a composição destas com o difeomorfismo

$$
T^{\epsilon}: R^{\epsilon} \rightarrow Y:\left(x_{1}, x_{2}\right) \mapsto\left(\frac{x_{1}}{\epsilon}, \frac{x_{2}}{\epsilon}\right) .
$$

$\mathrm{Na}$ sequência, com algum abuso de notação denotaremos esta composição por $X\left(\frac{x_{1}}{\epsilon}, \frac{x_{2}}{\epsilon}\right) e$ $\theta\left(\frac{x_{1}}{\epsilon}, \frac{x_{2}}{\epsilon}\right)$ para todo $\left(x_{1}, x_{2}\right) \in R^{\epsilon}$. Com estas considerações podemos obter algumas estimativas sobre $R^{\epsilon}$ para $X$ e $\theta$, a saber

$$
\left\{\begin{aligned}
\|X\|_{L^{2}\left(R^{\epsilon}\right)}^{2} & =\int_{R^{\epsilon}}\left|X\left(\frac{x_{1}}{\epsilon}, \frac{x_{2}}{\epsilon}\right)\right|^{2} d x_{1} d x_{2} \\
& \leq \sum_{k=1}^{\frac{1}{\epsilon L}} \epsilon^{2} \int_{Y^{*}}|X(y, z)|^{2} d y d z \\
& \leq \frac{\epsilon}{L}\|X\|_{L^{2}\left(Y^{*}\right)}^{2},
\end{aligned}\right.
$$

e similarmente

$$
\left\{\begin{array}{c}
\|\theta\|_{L^{2}\left(R^{\epsilon}\right)}^{2}=\|\theta\|_{L^{2}\left(Y^{*}\right)}^{2} \\
\left\|\partial_{y} X\right\|_{L^{2}\left(R^{\epsilon}\right)}^{2}=\left\|\partial_{y} X\right\|_{L^{2}\left(Y^{*}\right)}^{2} \\
\left\|\partial_{y} \theta\right\|_{L^{2}\left(R^{\epsilon}\right)}^{2}=\left\|\partial_{y} \theta\right\|_{L^{2}\left(Y^{*}\right)}^{2}
\end{array}\right.
$$

Observação 4.1.2. Podemos resolver os problemas (4.14) e (4.16) aplicando o teorema de Lax-Milgram para a forma elíptica

$$
a_{Y^{*}}(\varphi, \phi)=\int_{Y^{*}} \nabla_{y, z} \varphi \cdot \nabla_{y, z} \phi d y d z, \forall \varphi, \phi \in H^{1}\left(Y^{*}\right)
$$

sobre o conjunto $V=V_{Y^{*} / \mathbb{R}}$ onde

$$
V_{Y^{*}}=\left\{\varphi \in H^{1}\left(Y^{*}\right) \mid \varphi(0, z)=\varphi(L, z)\right\}
$$

De fato, a seguinte quantidade

$$
\|\varphi\|_{V}=\left(\int_{Y^{*}}|\nabla \varphi|^{2} d y d z\right)^{\frac{1}{2}}
$$

define uma norma sobre $V$. 


\subsection{Corretor de Primeira Ordem}

Nesta seção definimos o corretor de primeira ordem $\kappa^{\epsilon}$ e demonstramos um teorema de aproximação para $\kappa^{\epsilon}$ e $u^{\epsilon}-u_{0}$ na norma $\||\cdot|\|_{H^{1}\left(\Omega^{\epsilon}\right)}$.

Definição 4.2.1. Definimos o corretor de primeira ordem por

$$
\kappa^{\epsilon}\left(x_{1}, x_{2}\right)=-\epsilon X\left(\frac{x_{1}}{\epsilon}, \frac{x_{2}}{\epsilon}\right) \frac{d u_{0}}{d x_{1}}\left(x_{1}\right),\left(x_{1}, x_{2}\right) \in R^{\epsilon}
$$

Teorema 4.2.1. Seja $u^{\epsilon}$ a solução do problema (4.2) com $f^{\epsilon} \in L^{2}\left(R^{\epsilon}\right)$ satisfazendo

$$
\|\left.\left|f^{\epsilon}\right|\right|_{L^{2}\left(R^{\epsilon}\right)} \leq C
$$

para algum $C>0$ independente de $\epsilon$. Considere a família de funções $\widehat{f^{\epsilon}} \in L^{1}(0,1)$ definidas por

$$
\widehat{f} \epsilon\left(x_{1}\right)=\epsilon^{-1} \int_{0}^{\epsilon g\left(x_{1} / \epsilon\right)} f^{\epsilon}\left(x_{1}, x_{2}\right) d x_{2} .
$$

Se $\widehat{f} \epsilon \widehat{f}$, então

$$
\lim _{\epsilon \rightarrow 0} \mid\left\|u^{\epsilon}-u_{0}-\kappa^{\epsilon}\right\|_{H^{1}\left(R^{\epsilon}\right)}=0
$$

onde $\kappa^{\epsilon}$ é o corretor de primeira ordem de $u^{\epsilon}$ e $u_{0} \in H^{2}(0,1) \cap C^{1}(0,1)$ é a única solução da equação (4.12) com

$$
f_{0}=\frac{1}{\widehat{g}} \widehat{f}
$$

Prova: Pela formulação variacional de (4.2), temos

$$
a_{\epsilon}\left(\varphi, u^{\epsilon}\right)=\left(\varphi, f^{\epsilon}\right)_{\epsilon}, \forall \varphi \in H^{1}\left(R^{\epsilon}\right) .
$$

Assim, notando que $u_{0}+\kappa^{\epsilon} \in H^{1}\left(R^{\epsilon}\right)$, obtemos por simetria de $a_{\epsilon}$

$$
\left\{\begin{aligned}
\left\|\left|u^{\epsilon}-u_{0}-\kappa^{\epsilon}\right|\right\|_{H^{1}\left(R^{\epsilon}\right)}^{2}= & a_{\epsilon}\left(u^{\epsilon}-u_{0}-\kappa^{\epsilon}, u^{\epsilon}-u_{0}-\kappa^{\epsilon}\right) \\
& a_{\epsilon}\left(u^{\epsilon}, u^{\epsilon}-u_{0}-\kappa^{\epsilon}\right)-a_{\epsilon}\left(u_{0}+\kappa^{\epsilon}, u^{\epsilon}\right)+a_{\epsilon}\left(u_{0}+\kappa^{\epsilon}, u_{0}+\kappa^{\epsilon}\right) \\
& \left(u^{\epsilon}-2\left(u_{0}+\kappa^{\epsilon}\right), f^{\epsilon}\right)_{\epsilon}+a_{\epsilon}\left(u_{0}+\kappa^{\epsilon}, u_{0}+\kappa^{\epsilon}\right)
\end{aligned}\right.
$$

Fazendo a mudânça de variáveis $(x, y) \rightarrow(x, y / \epsilon)$ no Teorema 3.4.1, podemos concluir que

$$
\epsilon^{-1}\left\|u^{\epsilon}-u_{0}\right\|_{L^{2}\left(R^{\epsilon}\right)} \rightarrow 0 \text { quando } \epsilon \rightarrow 0
$$

consequentemente, $\left.||\left|u^{\epsilon}-u_{0}\right|\right|_{L^{2}\left(R^{\epsilon}\right)} \stackrel{\epsilon \rightarrow 0}{\rightarrow} 0$. Portanto

$$
\left(u^{\epsilon}-u_{0}, f^{e}\right)_{\epsilon} \leq \||| u^{\epsilon}-\left.\left.u_{0}||\right|_{L^{2}\left(R^{\epsilon}\right)}||\left|f^{\epsilon}\right|\right|_{L^{2}\left(R^{\epsilon}\right)} \rightarrow 0, \text { quando } \epsilon \rightarrow 0 .
$$


De (4.17) também obtemos

$$
\left(\kappa^{\epsilon}, f^{\epsilon}\right)_{\epsilon} \leq \epsilon^{-1}\left\|\kappa^{\epsilon}\right\|_{L^{2}\left(R^{\epsilon}\right)}\left\|f^{\epsilon}\right\|_{L^{2}\left(R^{\epsilon}\right)} \leq \frac{\epsilon C}{L^{1 / 2}}\|X\|_{L^{2}\left(Y^{*}\right)}\left\|\frac{d u_{0}}{d x_{1}}\right\|_{L^{\infty}(0,1)} \rightarrow 0, \text { quando } \epsilon \rightarrow 0 .
$$

Agora, como $\widehat{f}^{\epsilon} \rightarrow \widehat{f}$ em $L^{2}(0,1)$ obtemos

$$
\left\{\begin{aligned}
\left(u_{0}, f^{\epsilon}\right)_{\epsilon} & =\epsilon^{-1} \int_{0}^{1} u_{0}\left(x_{1}\right) \int_{0}^{\epsilon g\left(x_{1} / \epsilon\right)} f^{\epsilon}\left(x_{1}, x_{2}\right) d x_{2} d x_{1} \\
& =\int_{0}^{1} u_{0}\left(x_{1}\right) \widehat{f} \epsilon\left(x_{1}\right) d x_{1} \\
& \rightarrow \widehat{g} \int_{0}^{1} u_{0}\left(x_{1}\right) f_{0}\left(x_{1}\right) d x_{1}, \quad \text { quando } \epsilon \rightarrow 0 .
\end{aligned}\right.
$$

Portanto, obtemos de (4.24), (4.25), (4.26) e (4.8) que

$$
\left(u^{\epsilon}-2\left(u_{0}+\kappa^{\epsilon}\right), f^{\epsilon}\right)_{\epsilon} \stackrel{\epsilon \rightarrow 0}{\rightarrow}\left(u_{0}, f_{0}\right)_{0} .
$$

A seguir mostraremos que

$$
a_{\epsilon}\left(u_{0}+\kappa^{\epsilon}, u_{0}+\kappa^{\epsilon} \rightarrow a_{0}\left(u_{0}, u_{0}\right) \text { quando } \epsilon \rightarrow 0 .\right.
$$

Primeiro calculamos o limite de

$$
\begin{gathered}
a_{\epsilon}\left(u_{0}+\kappa^{\epsilon}, u_{0}\right)=\epsilon^{-1} \int_{R^{\epsilon}}\left\{\nabla\left(u_{0}+\kappa^{\epsilon}\right) \cdot \nabla u_{0}+\left(u_{0}+\kappa^{\epsilon}\right) u_{0}\right\} d x_{1} d x_{2} \\
=\epsilon^{-1} \int_{R^{\epsilon}}\left\{\frac{d u_{0}}{d x_{1}}-\partial_{y} X\left(\frac{x_{1}}{\epsilon}, \frac{x_{2}}{\epsilon}\right) \frac{d u_{0}}{d x_{1}}-X\left(\frac{x_{1}}{\epsilon}, \frac{x_{2}}{\epsilon}\right) \frac{d^{2} u_{0}}{d x_{1}^{2}}\right\} \frac{d u_{0}}{d x_{1}} d x_{1} d x_{2} \\
+\epsilon^{-1} \int_{R^{\epsilon}}\left(u_{0}+\kappa^{\epsilon}\right) u_{0} d x_{1} d x_{2} \\
=\epsilon^{-1} \int_{R^{\epsilon}} \frac{d u_{0}^{2}}{d x_{1}}\left\{1-\partial_{y} X\left(\frac{x_{1}}{\epsilon}, \frac{x_{2}}{\epsilon}\right)\right\} d x_{1} d x_{2}+\epsilon^{-1} \int_{R^{\epsilon}}\left|u_{0}\right|^{2} d x_{1} d x_{2} \\
-\epsilon^{-1} \int_{R^{\epsilon}}\left\{\epsilon X\left(\frac{x_{1}}{\epsilon}, \frac{x_{2}}{\epsilon}\right) \frac{d u_{0}}{d x_{1}} \frac{d^{2} u_{0}}{d x_{1}^{2}}+\epsilon X\left(\frac{x_{1}}{\epsilon}, \frac{x_{2}}{\epsilon}\right) u_{0} \frac{d u_{0}}{d x_{1}}\right\} d x_{1} d x_{2}
\end{gathered}
$$

quando $\epsilon \rightarrow 0$. Assim,

$$
\epsilon^{-1} \int_{R^{\epsilon}} \frac{d u_{0}^{2}}{d x_{1}}\left\{1-\partial_{y} X\left(\frac{x_{1}}{\epsilon}, \frac{x_{2}}{\epsilon}\right)\right\} d x_{1} d x_{2}=\int_{0}^{1} \int_{0}^{g\left(x_{1} / \epsilon\right)} \frac{d u_{0}^{2}}{d x_{1}}\left\{1-\partial_{y} X\left(\frac{x_{1}}{\epsilon}, z\right)\right\} d z d x_{1},
$$

e

$$
\Phi(y)=\int_{0}^{g(y)}\left\{1-\partial_{y} X(y, z)\right\} d z
$$


é uma função L-periódica, obtemos que

$$
\begin{aligned}
\epsilon^{-1} \int_{R^{\epsilon}} \frac{d u_{0}^{2}}{d x_{1}}\left\{1-\partial_{y} X\left(\frac{x_{1}}{\epsilon}, \frac{x_{2}}{\epsilon}\right)\right\} d x_{1} d x_{2} & \rightarrow \int_{0}^{1} \frac{d u_{0}^{2}}{d x_{1}} \frac{1}{L} \int_{0}^{L} \int_{0}^{g(y)}\left\{1-\partial_{y} X(y, z)\right\} d z d y d x_{1} \\
& =\widehat{g} \int_{0}^{1} q_{0} \frac{d u_{0}^{2}}{d x_{1}} d x_{1} \text { quando } \epsilon \rightarrow 0 .
\end{aligned}
$$

Note também que

$$
\epsilon^{-1} \int_{R^{\epsilon}}\left|u_{0}\right|^{2} d x_{1} d x_{2} \rightarrow \widehat{g} \int_{0}^{1}\left|u_{0}\right|^{2} d x_{1} \text { quando } \epsilon \rightarrow 0 .
$$

Como $u_{0}$ não depende de $x_{2}$ segue de (4.17) que

$$
-\epsilon^{-1} \int_{R^{\epsilon}}\left\{\epsilon X\left(\frac{x_{1}}{\epsilon}, \frac{x_{2}}{\epsilon}\right) \frac{d u_{0}}{d x_{1}} \frac{d^{2} u_{0}}{d x_{1}^{2}}+\epsilon X\left(\frac{x_{1}}{\epsilon}, \frac{x_{2}}{\epsilon} u_{0} \frac{d u_{0}}{d x_{1}}\right)\right\} d x_{1} d x_{2} \rightarrow 0, \text { quando } \epsilon \rightarrow 0 \text {. }
$$

Assim, temos de (4.30), (4.31), (4.32) e (4.7) que

$$
a_{\epsilon}\left(u_{0}+\kappa^{\epsilon}, \kappa^{\epsilon}\right) \rightarrow 0, \text { quando } \epsilon \rightarrow 0 .
$$

Finalmente, argumentando como em (4.29), obtemos de (4.17) e (4.18) que

$$
a_{\epsilon}\left(u_{0}+\kappa^{\epsilon}, \kappa^{\epsilon}\right) \rightarrow 0, \text { quando } \epsilon \rightarrow 0,
$$

o que demonstra a afirmação (4.28).

Portanto, de acordo com (4.23) obtemos

$$
\|\left.\left|u^{\epsilon}-u_{0}-\kappa^{\epsilon}\right|\right|_{H^{1}\left(R^{\epsilon}\right)} ^{2} \stackrel{\epsilon \rightarrow 0}{\rightarrow} a_{0}\left(u_{0}, u_{0}\right)-\left(u_{0}, f_{0}\right)_{0}=0
$$

e isto completa a prova.

\subsection{Corretor de Segunda Ordem}

Nesta seção apresentamos uma estimativa do erro quando trocamos $u^{\epsilon}$ pela aproximação de segunda ordem

$$
\mathcal{W}^{\epsilon}=u_{0}\left(x_{1}\right)-\epsilon X\left(\frac{x_{1}}{\epsilon}, \frac{x_{2}}{\epsilon}\right) \frac{d u_{0}}{d x_{1}}\left(x_{1}\right)+\epsilon \theta\left(\frac{x_{1}}{\epsilon}, \frac{x_{2}}{\epsilon}\right) \frac{d^{2} u_{0}}{d x_{1}^{2}}\left(x_{1}\right),
$$

com respeito a norma $\mid\|\cdot\| \|_{H^{1}\left(R^{\epsilon}\right)}$ definida na seção 2 , onde $u_{0}$ é solução da equação homogeneizada (4.12), e as funções $X$ e $\theta$ foram definidas pelos problemas auxiliares (4.11) e (4.16) sobre a região $Y^{*}$ que são convenientemente extendidas para o domínio fino $R^{\epsilon}$

Para a aproximação de segunda ordem tem-se o seguinte resultado. 
Teorema 4.3.1. Seja $R^{\epsilon}$ o domínio fino definido em (4.1) e seja $u^{\epsilon}$ a solução do problema (4.2) $\operatorname{com} f^{\epsilon}\left(x_{1}, x_{2}\right)=f\left(x_{1}\right), f \in W^{2, \infty}(0,1)$.

Nestas condições, se $\mathcal{W}^{\epsilon}$ é dado por (4.34), então existe uma constante $K$ independente de $\epsilon$ tal que

$$
\left\|\left|u^{\epsilon}-\mathcal{W}^{\epsilon}\right|\right\|_{H^{1}\left(R^{\epsilon}\right)} \leq K \epsilon^{1 / 2}
$$

No que segue, apresentamos o esboço de uma prova. Uma versão completa da demonstração deste teorema se encontra em [18].

Seja $\phi^{\epsilon}:=u^{\epsilon}-\mathcal{W}^{\epsilon}$. Usando (4.11), (4.2), (4.12) e (4.16) pode-se demonstrar que $\phi^{\epsilon}$ satisfaz o seguinte problema de valor de fronteira

$$
\left\{\begin{aligned}
-\Delta \phi^{\epsilon}+\phi^{\epsilon} & =\epsilon F^{\epsilon} \text { em } R^{\epsilon} \\
\frac{\partial \phi^{\epsilon}}{\partial N^{\epsilon}} & =\epsilon^{2} H^{\epsilon} N_{1}^{\epsilon} \text { em } \partial R^{\epsilon}
\end{aligned}\right.
$$

onde

$$
\begin{aligned}
F^{\epsilon}\left(x_{1}, x_{2}\right) & =-\left[\frac{d^{3} u_{0}}{d x^{3}}\left(x_{1}\right)\left(X\left(\frac{x_{1}}{\epsilon}, \frac{x_{2}}{\epsilon}\right)-2 \partial_{y} \theta\left(\frac{x_{1}}{\epsilon}, \frac{x_{2}}{\epsilon}\right)\right)-X\left(\frac{x_{1}}{\epsilon}, \frac{x_{2}}{\epsilon}\right) \frac{d u_{0}}{d x}\left(x_{1}\right)\right] \\
& -\epsilon\left[\theta\left(\frac{x_{1}}{\epsilon}, \frac{x_{2}}{\epsilon}\right)\left(\frac{d^{2} u_{0}}{d x^{2}}\left(x_{1}\right)-\frac{d^{4} u_{0}}{d x^{4}}\left(x_{1}\right)\right)\right] \text { em } R^{\epsilon}
\end{aligned}
$$

$\mathrm{e}$

$$
H^{\epsilon}\left(x_{1}, x_{2}\right)=-\theta\left(\frac{x_{1}}{\epsilon}, \frac{x_{2}}{\epsilon}\right) \frac{d^{3} u_{0}}{d x^{3}}\left(x_{1}\right) \text { em } \partial R^{\epsilon} .
$$

Depois, consideramos a formulação variacional de (4.35) que é: encontrar $\phi^{\epsilon} \in H^{1}\left(R^{\epsilon}\right)$ tal que

e com isto obtemos

$$
a_{\epsilon}\left(\phi^{\epsilon}, \varphi\right)=\int_{R^{\epsilon}} F^{\epsilon} \varphi d x+\epsilon \int_{\partial R^{\epsilon}} H^{\epsilon} N_{1}^{\epsilon} \varphi d S
$$

$$
\left\|| | \phi ^ { \epsilon } \left|\left\|_{H^{1}\left(R^{\epsilon}\right)}^{2}=\left|a_{\epsilon}\left(\phi^{\epsilon}, \phi^{\epsilon}\right)\right| \leq\right\| \phi^{\epsilon}\left\|_{L^{2}\left(R^{\epsilon}\right)}\right\| f^{\epsilon}\left\|_{L^{2}\left(R^{\epsilon}\right)}+\epsilon\right\| \phi^{\epsilon}\left\|_{L^{2}\left(\partial R^{\epsilon}\right)}\right\| H^{\epsilon} N_{1}^{\epsilon} \|_{L^{2}\left(\partial R^{\epsilon}\right)} .\right.\right.
$$

Fazendo algumas estimativas sobre a norma $L^{2}\left(R^{\epsilon}\right)$ de $X, \theta$ e $\partial_{y} \theta$ obtemos

$$
\left\|\left|\phi^{\epsilon}\right|\right\|_{H^{1}\left(R^{\epsilon}\right)}^{2} \leq \epsilon^{1 / 2} K_{0}\left\|\phi^{\epsilon}\right\|_{L^{2}\left(R^{\epsilon}\right)}+\epsilon K_{1}\left\|\phi^{\epsilon}\right\|_{L^{2}\left(R^{\epsilon}\right)} .
$$

Para concluir o resultado, basta conferir que existe $C>0$ tal que

$$
\|\phi\|_{L^{2}\left(\partial R^{\epsilon}\right)} \leq C_{1} \epsilon^{1 / 2}\|\phi\|_{H^{1}\left(R^{\epsilon}\right)} .
$$

Uma forma de obter (4.40) é calcular $\|\phi\|_{L^{2}\left(\partial R^{\epsilon}\right)}^{2}$ com o auxílio de uma extensão $M=$ $\left(M_{1}, M_{2}\right) \in C^{1}\left(\overline{Y^{*}}\right)$ do campo $N$ de vetores normais unitários definido em $\partial Y^{*}$ e usando o teorema do divergente. 


\section{Considerações finais}

Esta dissertação foi motivada pelo estudo do comportamento assintótico de soluções de problemas de valores de fronteira que estão definidos em domínios que variam com um parâmetro $\epsilon$. Problemas semelhantes a este aparecem naturalmente quando se estuda a modelagem de fenômenos em que tem-se imprecisão quanto ao domínio das funções envolvidas, ou quando se estuda problemas de homogeneização de estruturas reticuladas formadas por materiais compostos.

Neste trabalho tentamos destacar um tipo de abordagem possível para se analisar a convergência dos resolventes bem como a convergência dos semigrupos dos operadores associados quando estes são definidos em domínios oscilantes. Esta abordagem consiste em usar técnicas de homogeneização para se obter a convergência dos resolventes e depois, dos semigrupos.

Inspirado neste uso da teoria de homogeneização para a análise da convergência do resolvente, dos auto-valores e auto-vetores de operadores diferencias, pretendemos continuar os estudos com os operadores associados ao problema parabólico, oriundo de (6). Nossa intenção é melhorar as taxas de convergência de atratores dos semigrupos semilineares oriundos dos operadores parabólicos com inspiração nas estimativas de erro discutidas no Capítilo 4. Pretendemos também abordar o caso em que tem-se o domínio fixo e os operadores são da forma divergente constituído por funções periódicas que dependem de um parâmetro $\epsilon$ que tende a zero, aumentando a oscilação. Dessa forma estaremos contribuindo com o estudo de sistemas dinâmicos não lineares associados a problemas de valor inicial com algum tipo de oscilação que varia com um parâmetro. 


\section{Referências Bibliográficas}

[1] J. M. Arrieta and A. N. Carvalho; Spectral Convergence and nonlinear dynamics of reaction-difusion equations under perturbations of the domain, J. Differential Equations 199, 143-178 (2004).

[2] J. M. Arrieta, A. N. Carvalho and G. Lozada-Cruz; Dynamics in dumbbell domains I. Continuity of the set of equilibria, J. Differential Equations 231, 551-597 (2006).

[3] J. M. Arrieta, A. N. Carvalho and G. Lozada-Cruz; Dynamics in dumbbell domains II. The limiting problem, J. Differential Equations 247, 174-202 (2009).

[4] J. M. Arrieta, A. N. Carvalho and G. Lozada-Cruz; Dynamics in dumbbell domains III. Continuity of attractors, J. Differential Equations 247, 225-259 (2009).

[5] H. Brezis, Analyse Fonctionnelle: Théorie et applications. Collection Mathématiques appliquées pour la maîtrise, (2) (1987).

[6] A. N. Carvalho and S. Piskarev, A general approximation scheme for attractors of abstract parabolic problems, Numerical Functional Analysis and Optimization 27 (7-8) 785 - 829 (2006)

[7] G. B. Folland, Real Analysis: Modern techniques and their applications, Pure \& Applied Mathematics, Second edition, John Wiley \& Sons.

[8] J. K. Hale Asymptotic Behavior of Dissipative Systems, Mathematical Surveys and Monographs 25 (American Mathematical Society, Providence, RI) (1988).

[9] J. K. Hale, L. Magalhães and W. M. Oliva, Dynamics in Infinite Dimensions, Applice Mathematical Sciences 47, Second Edition, Springer Verlag (2002).

[10] J. K. Hale and G. Raugel, Lower semicontinuity of attractors of gradient systems and applications, Ann. Mat. Pura Appl. 154 (4) (1989), 281-326. 
[11] D. Henry, Geometric Theory of Semilinear Parabolic Equations, Lecture Notes in Mathematics 840, Springer-Verlag, Berlin, 1981.

[12] D. Cioranescu and J. Saint J. Paulin; Homogenization of Reticulated Structures, Springer Verlag (1980).

[13] D. Cioranescu and P. Donato; An Introduction to Homogenization, Oxford (1999).

[14] Pazy, A.,Semigroups of Linear Operators and Applications to Partial Differential Equations, Springer Verlag, New York, (1983)

[15] L. Tartar; Problèmmes d’homogénéisation dans les équations aux dérivées partielles, Cours Peccot, Collège de France (1977).

[16] L. Tartar; Quelques remarques sur l'homegénéisation, Function Analysis and Numerical Analysis, Proc. Japan-France Seminar 1976, ed. H. Fujita, Japanese Society for the Promotion of Science, 468-482 (1978).

[17] José M. Arrieta, Alexandre N. Carvalho, Marcone C. Pereira and Ricardo P. da Silva, Nonlinear parabolic problems in thin domains with a highly oscillatory boundary , Nonlinear Analysis 74 5111-5132 (2011).

[18] Marcone C. Pereira and Ricardo P. da Silva, Error estimatives and homogenization for a Neumann problem in highly oscillating thin domain. Submitted

[19] Taylor, Angus Ellis 1911-Introduction to functional analysis / Angus E. Taylor, David C. Lay, John Wiley (1958).

[20] Alexandre N. Carvalho, José M. Arrieta, Aníbal R. Bernal , Attractors of Parabolic Problems with Nonlinear Boundary Conditions. Uniform Bounds. June 24, 2003. 